\title{
Electrochemical Synthesis of Cyanoformamides from Trichloroacetonitrile and Secondary Amines Mediated by the $B_{12}$ Derivative
}

Mohammad Moniruzzaman, Yoshio Yano, Toshikazu Ono, Kenji Imamura," ${ }^{*}$ oshihito Shiota, ${ }^{\#}$ Kazunari Yoshizawa, ${ }^{\#}$ Yoshio Hisaeda*, and Hisashi Shimakoshi*

Department of Chemistry and Biochemistry, Graduate School of Engineering, Kyushu University, Motooka, Fukuoka 819-0395, Japan.

E-mail: yhisatcm@mail.cstm.kyushu-u.ac.jp, shimakoshi@mail.cstm.kyushu-u.ac.jp.

\#Institute for Materials Chemistry and Engineering, Kyushu University, Fukuoka 819-0395, Japan 


\section{Contents}

1. General procedures for electrode preparation S1-S2

2. Catalyst used in this study S2

3. Experiment setup for general procedure of bulk s3 electrolysis
Experiment setup for gram-scale electrolysis

$\begin{array}{llr}\text { 4. Experiment setup for gram-scale electrolysis } & \text { S3 } \\ \text { 5. Cyclic voltammetry studies } & \text { S4-S5 }\end{array}$

6. Mechanistic study S5-S27

7. X-ray Crystallography S28-S31

8. $\mathrm{Cl}^{-}$quantification by mercury(II) thiocyanate method $\mathrm{S} 32$

9. ${ }^{1} \mathrm{H}$ and ${ }^{13} \mathrm{C}$ NMR spectra S33-S73

10. ${ }^{19} \mathrm{~F}$ NMR spectra $\quad \mathrm{S} 74$

11. References $\quad$ S75-S76 


\section{General procedures for electrode preparation}

Materials used for setup: Platinum wire from Nilaco Corporation, zinc plate from Nilaco Corporation, carbon felt from ECFRONTER, and $\mathrm{Ag} / \mathrm{AgCl}(3 \mathrm{M}, \mathrm{NaCl}$, product Code: WL 310, $\mathrm{RE}-1 \mathrm{~B})$ reference electrode, were purchased from ALS Co., Ltd. for the electrodes setup.

Reference electrode setup: The $\mathrm{Ag} / \mathrm{AgCl}(3 \mathrm{M}, \mathrm{NaCl})$ was inserted into a cell holder with an ion permeable tip and $3 \mathrm{M} \mathrm{NaCl}$ solution inside.
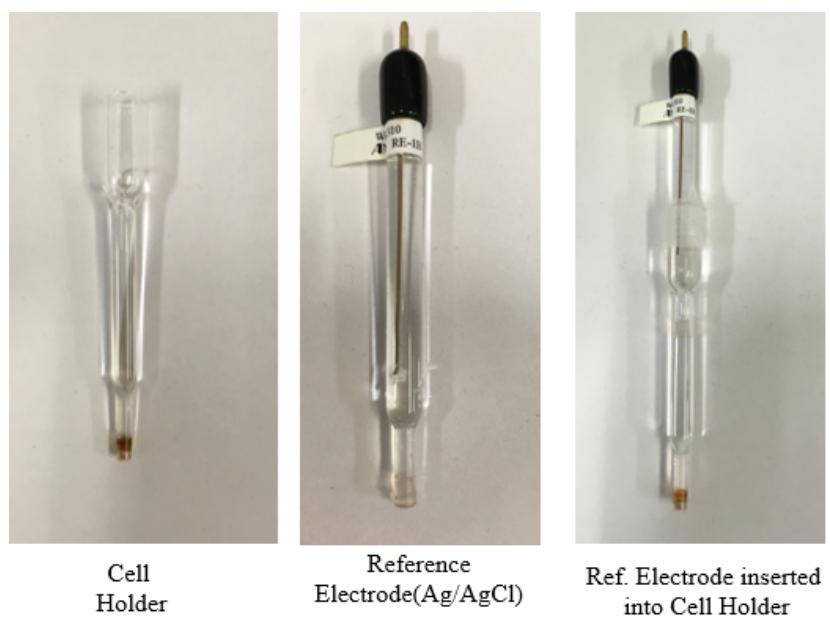

Figure S1. Homemade reference electrode setup. Photographs were taken by Mohammad Moniruzzaman.

Anode setup: A small rectangular $(\sim 4.3 \mathrm{~cm} \times 1.5 \mathrm{~cm})$ zinc plate was cut from a sheet and approximately $4 \mathrm{~cm}$ of a $9-\mathrm{cm}$ long platinum wire was coiled by wrapping around the zinc plate and the other end of the Pt wire was connected to the potentiostat.

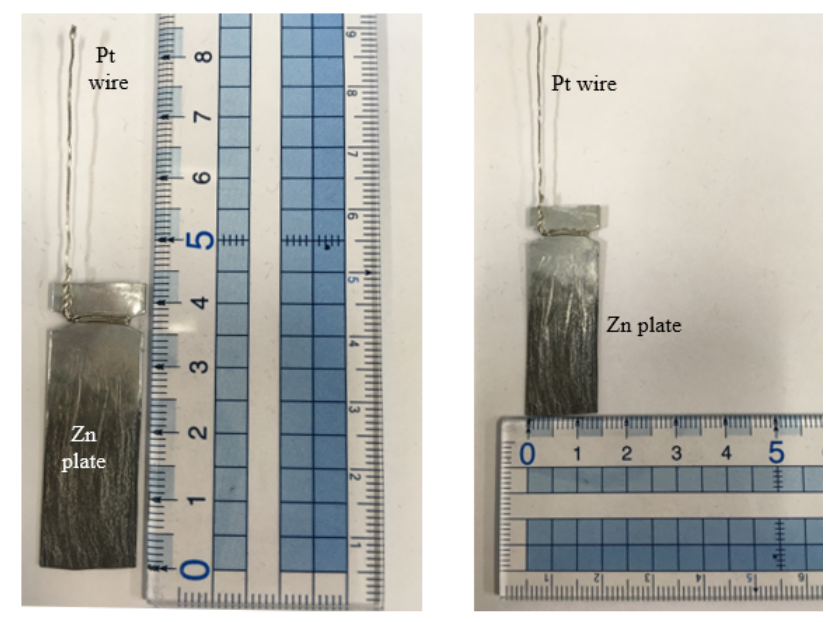

Figure S2. Setup of anode. 
Cathode setup: A small rectangular $(\sim 1.5 \mathrm{~cm} \times 1.0 \mathrm{~cm})$ carbon felt was cut from a sheet and clipped by a Pt wire, and the other end of the Pt wire was connected to the potentiostat.

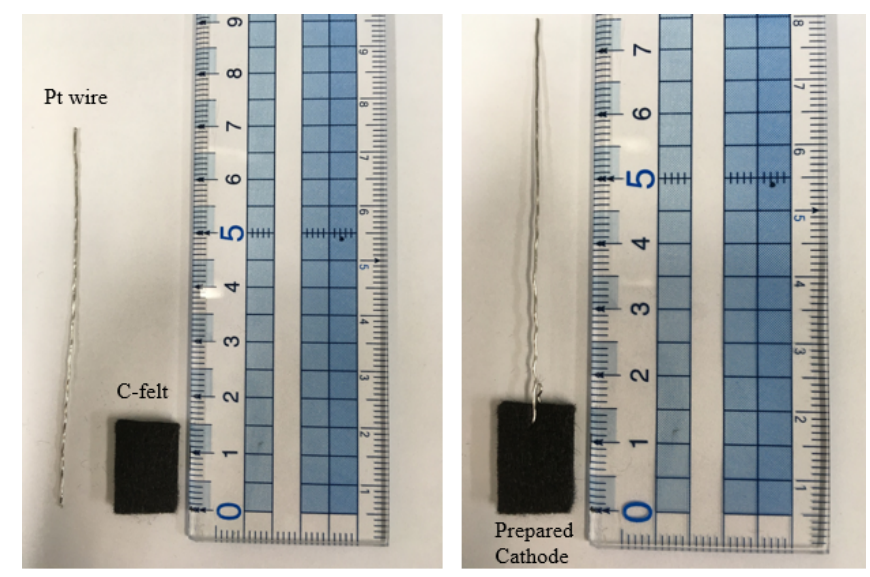

Figure S3. Setup of cathode.

Undivided cell: A simple 20-mL cylinder-type glass cell was used for the bulk electrolysis.

\section{Catalyst used in this study}

The catalyst $\mathbf{C} \mathbf{1}$ was used as the main catalyst. Besides $\mathbf{C} \mathbf{1}$, a costa-type cobalt complex (C2) was used to check the catalytic reaction.
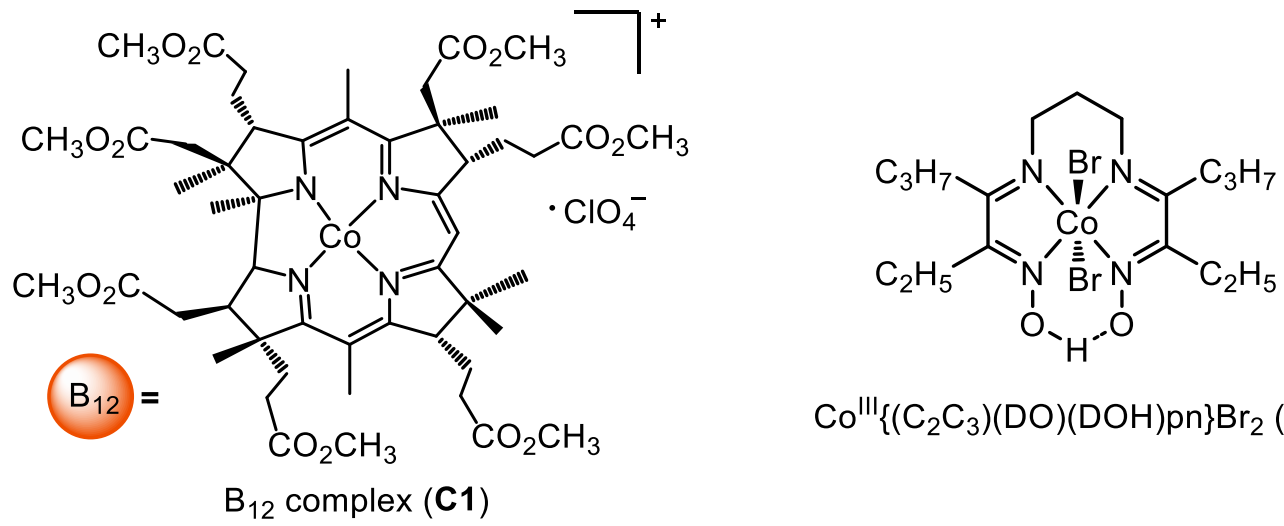

$\mathrm{Co}^{\prime \prime \prime}\left\{\left(\mathrm{C}_{2} \mathrm{C}_{3}\right)(\mathrm{DO})(\mathrm{DOH}) \mathrm{pn}\right\} \mathrm{Br}_{2}(\mathrm{C} 2)$ 
3. Experiment setup for general procedure of bulk electrolysis

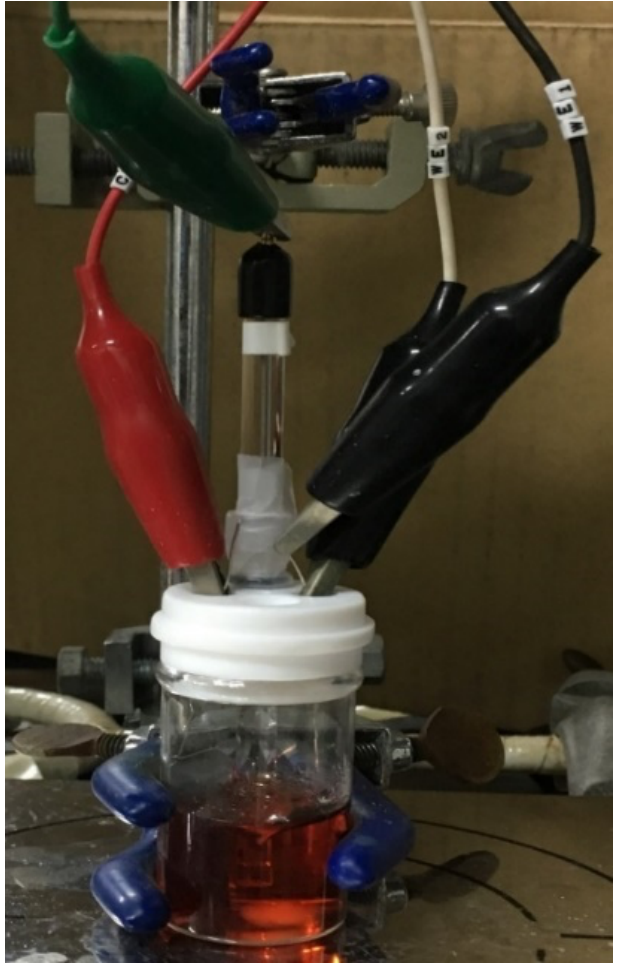

Figure S4. General bulk electrolysis setup.

4. Experiment setup for gram-scale electrolysis

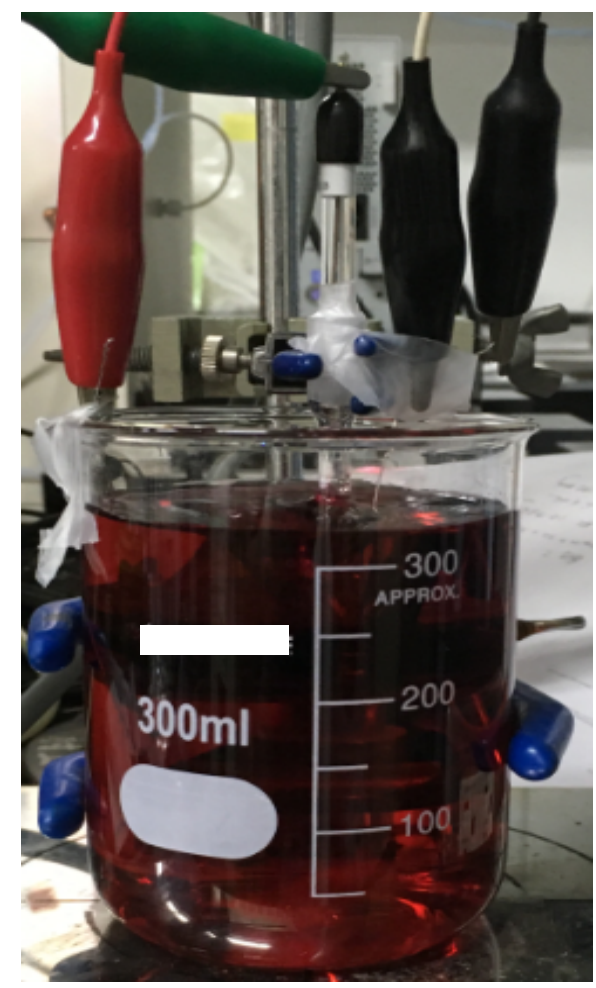

Figure S5. Gram-scale electrolysis setup. 


\section{Cyclic voltammetry studies}

\section{General information}

Cyclic voltammetry (CV) experiments were conducted in a 5-mL glass vial fitted with a glassy carbon working electrode, $\mathrm{Ag} / \mathrm{AgCl}(3 \mathrm{M}, \mathrm{NaCl})$ reference electrode, and a platinum coil counter electrode in $2 \mathrm{~mL}$ of anhydrous acetonitrile solvent. The solution of interest was purged with nitrogen for 5 minutes before data collection. The current was reported in $\mu \mathrm{A}$. Scan rate: $100 \mathrm{mV} / \mathrm{s},{ }^{n} \mathrm{Bu}_{4} \mathrm{NClO}_{4}$ as the supporting electrolyte $(0.1 \mathrm{M})$.

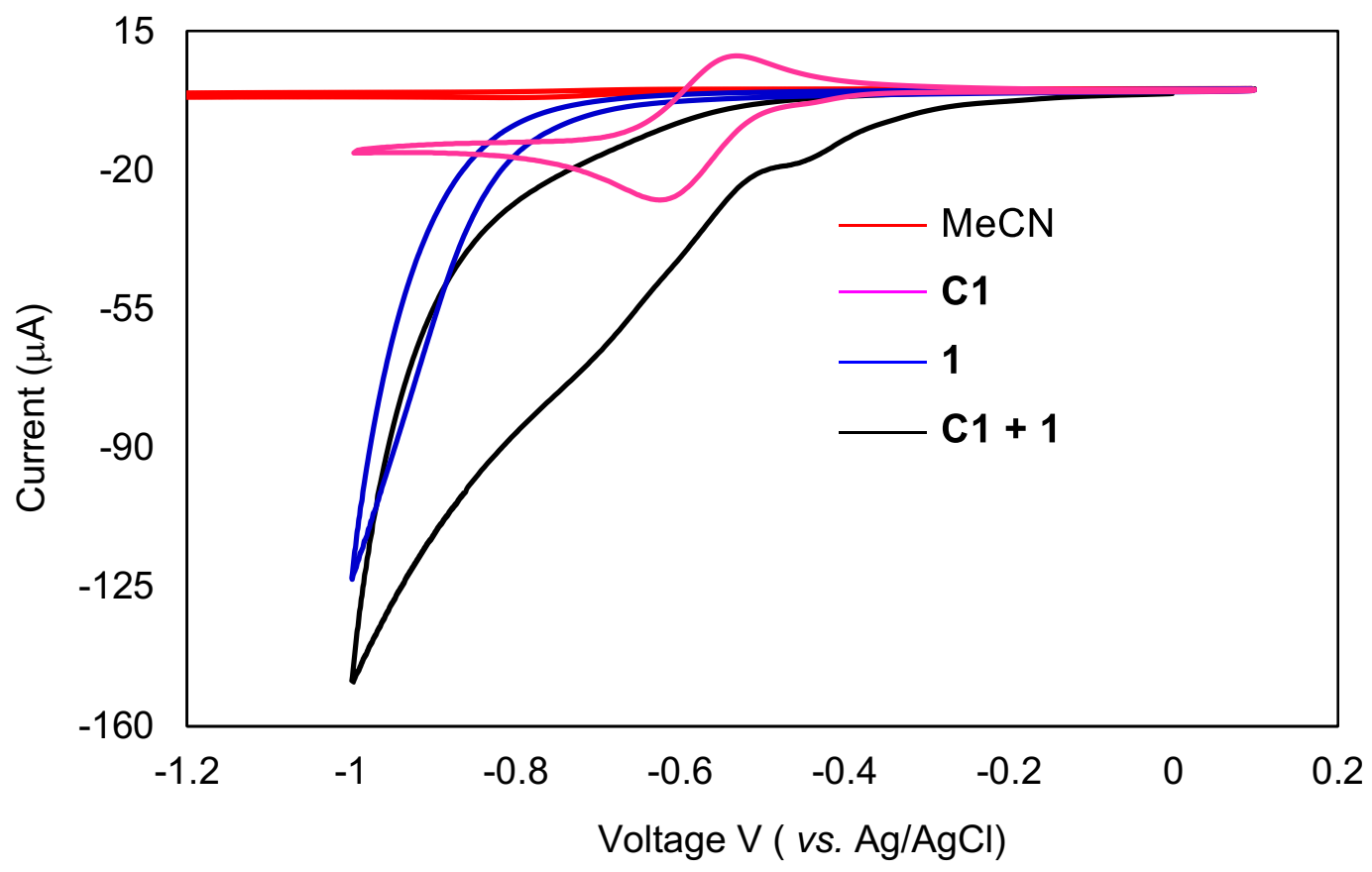

Figure S6. CV of MeCN solvent (red line), C1 (1.0 mM) (pink line), 1 (10.0 mM) (blue line), and $\mathbf{C} 1(1.0 \mathrm{mM})$ in the presence of $1(10.0 \mathrm{mM})$ (black line), in $0.1 \mathrm{M}$ of ${ }^{n} \mathrm{Bu}_{4} \mathrm{NClO}_{4} \mathrm{MeCN}$ solution in $\mathrm{N}_{2}$. Scan rate: $100 \mathrm{mV} / \mathrm{s}$. 


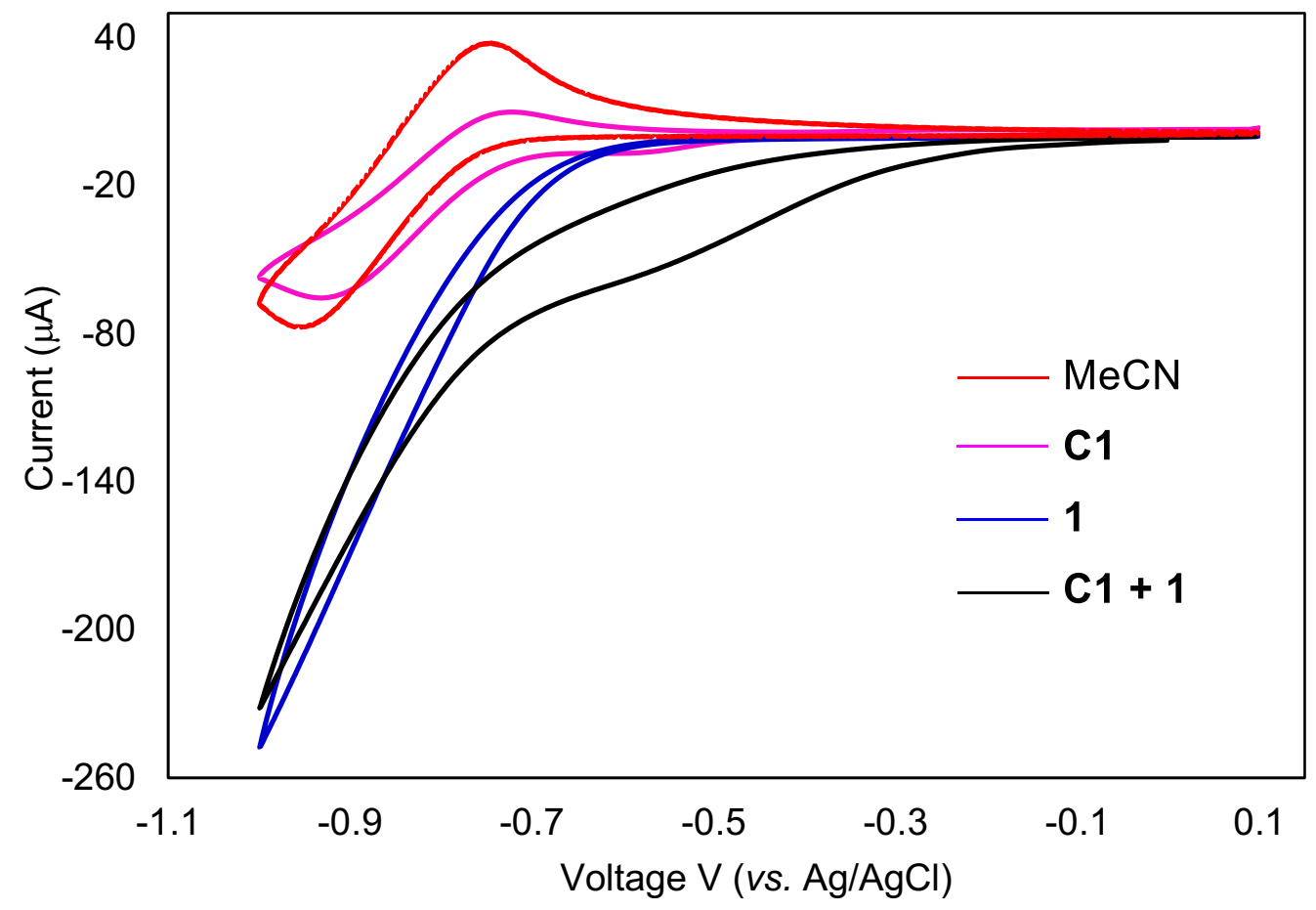

Figure S7. CV of MeCN solvent (red line), C1 (1.0 mM) (pink line), 1 (10.0 mM) (blue line), and $\mathbf{C} 1(1.0 \mathrm{mM})$ in the presence of $1(10.0 \mathrm{mM})$ (black line), in $0.1 \mathrm{M}$ of ${ }^{n} \mathrm{Bu}_{4} \mathrm{NClO}_{4} \mathrm{MeCN}$ solution in air. Scan rate: $100 \mathrm{mV} / \mathrm{s}$. 


\section{Mechanistic study}

a) Electrochemical alkylated complex formation

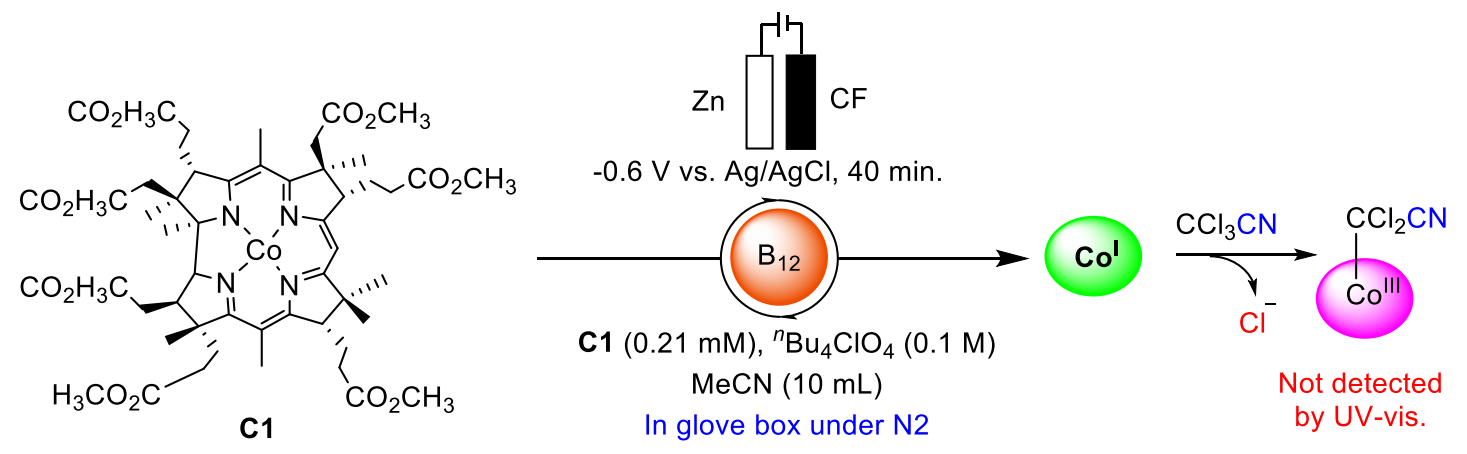

In $10 \mathrm{~mL}$ of anhydrous $\mathrm{MeCN}, \mathbf{C} 1(2.4 \mathrm{mg}, 0.21 \mathrm{mM})$ with ${ }^{n} \mathrm{Bu}_{4} \mathrm{NClO}_{4}(342.1 \mathrm{mg}, 0.1 \mathrm{M})$ was dissolved in a glovebox $\left(\mathrm{O}_{2}<1 \mathrm{ppm}\right)$ in the dark. After electrolysis at $-0.6 \mathrm{~V} v \mathrm{~s}$. $\mathrm{Ag} / \mathrm{AgCl}$ for 40 minutes, an excess amount of $1(50 \mu \mathrm{L}, 49.5 \mathrm{mM})$ was added to the reaction mixture and stirred for 2 minutes. After filtering with a syringe, a small portion of the reaction mixture was added to a UV-visible cell containing anhydrous $\mathrm{MeOH}$ and the cell was tightly caped in the glovebox under an $\mathrm{N}_{2}$ atmosphere. After wrapping with aluminium foil, the UV-cell was removed from the glovebox and the UV-visible spectrum immediately measured. After irradiation by visible light (halogen lamp, L42 filter, > $421 \mathrm{~nm}$ ) for 5 minutes, and the UVvisible spectrum was measured again. The typical UV-Vis. spectrum for the alkylated complex of $\mathrm{CCl}_{3} \mathrm{CN}$ was not detected.

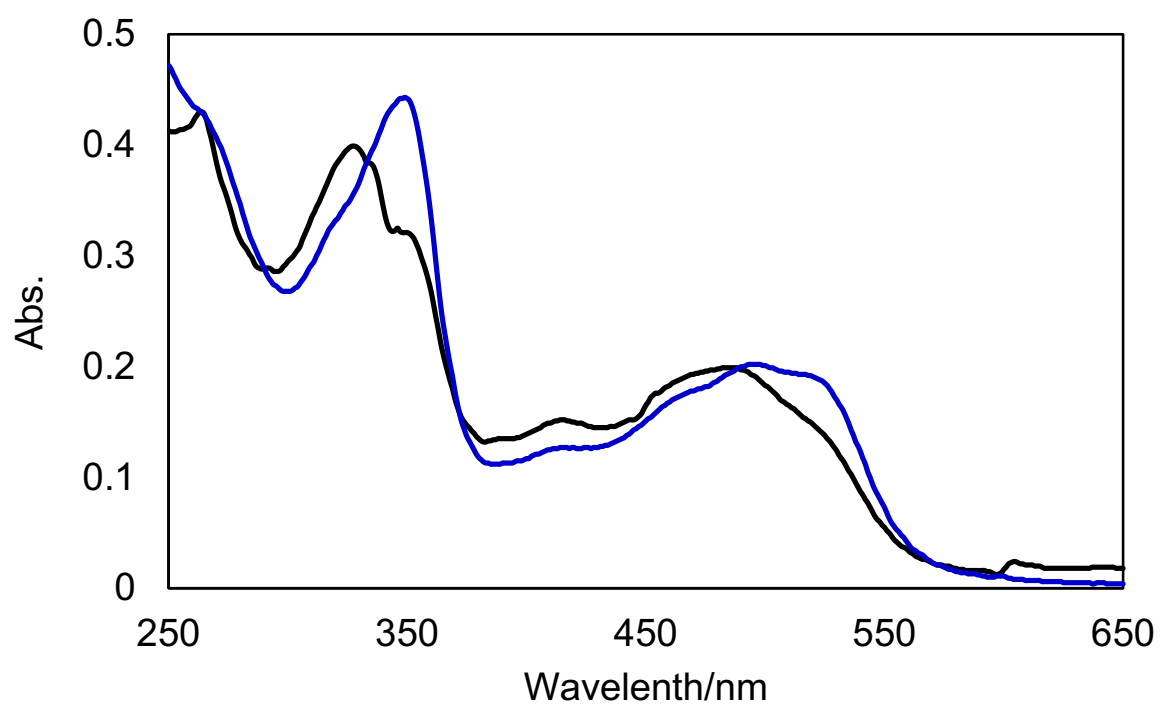

Figure S8. UV-vis spectrum of expected alkylated complex before irradiation by visible light (black line), and after irradiation by visible light (blue line) in $\mathrm{MeOH}$. 
b) Photochemical Alkylated Complex Formation

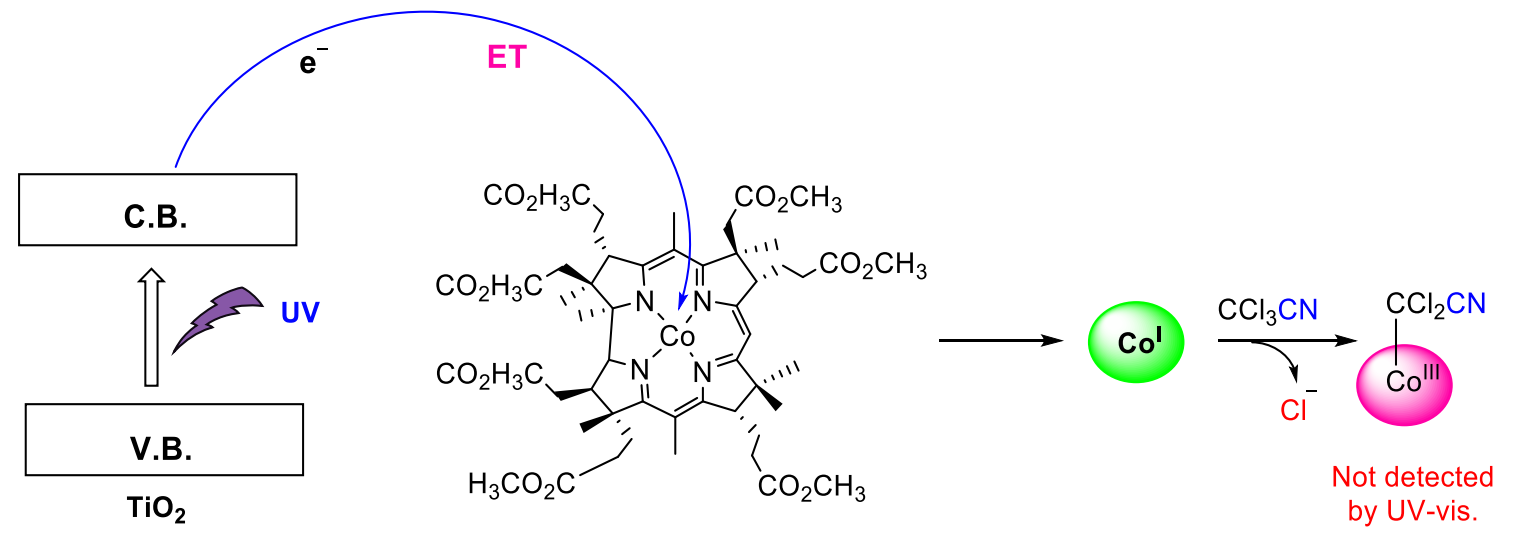

In a Schlenk tube $5 \mathrm{~mL}$ of MeCN solvent, titanium dioxide $(40.01 \mathrm{mg}, 0.1 \mathrm{M})$ and $1.50 \mathrm{mg}$ $(0.13 \mathrm{mM})$ of $\mathbf{C} 1$ were mixed well and purged with nitrogen gas for 15 minutes. After irradiation by UV light for 2 minutes in the dark, an excess amount of 1 ( $30 \mu \mathrm{L}, 59.4 \mathrm{mM}$ ) was added and shaken for 5 minutes. After filtration through a syringe filter, a small portion of the reaction mixture was added to methanol and the UV-visible spectrum and ESI-MS spectrum measured in the dark. After irradiation by visible light (halogen lamp, L42 filter, > $421 \mathrm{~nm}$ ) for 10 minutes, and the UV-visible spectrum was measured again. The typical UV-Vis. spectrum for the alkylated complex of $\mathrm{CCl}_{3} \mathrm{CN}$ was not detected.

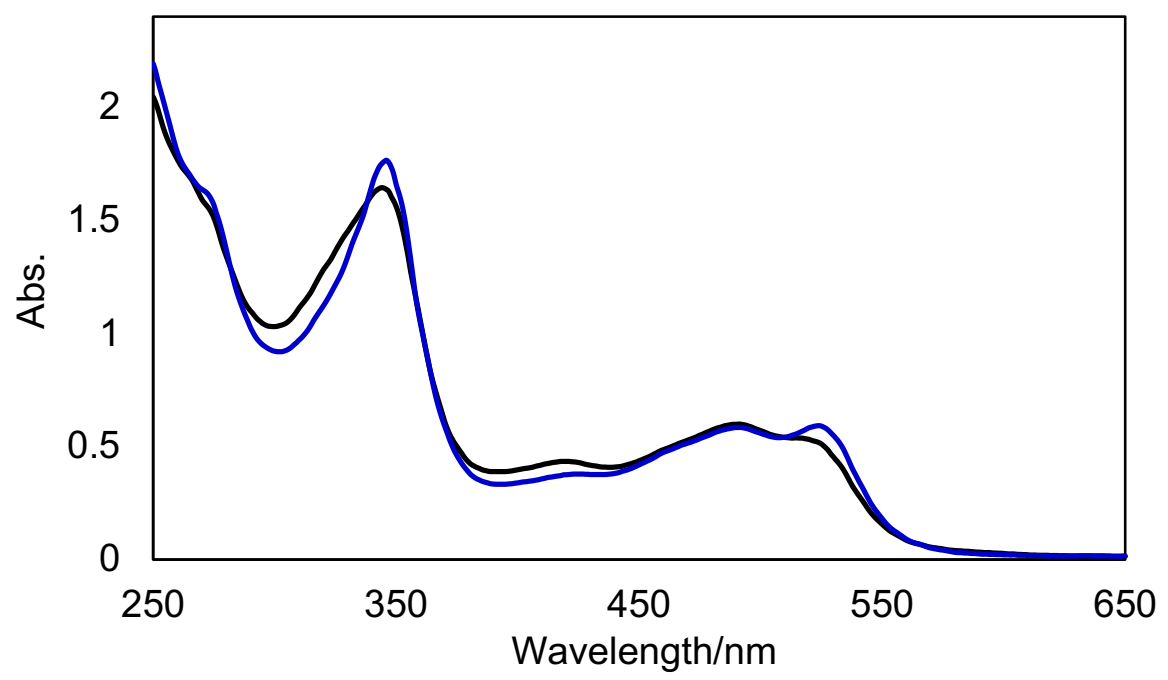

Figure S9. UV-vis spectrum of expected alkylated complex before irradiation by visible light (black line), and after irradiation by visible light (blue line) in $\mathrm{MeOH}$. 


\section{c) Calculation method}

Molecular orbital analysis in Figure 1 and Figure S10 were determined using the TPSS functional ${ }^{1}$ combined with the Grimme's D3 correction. ${ }^{2,3}$ We used the $(14 s 9 p 5 d) /[9 s 5 p 3 d]$ primitive set of Wachters-Hay ${ }^{4,5}$ supplemented with one polarization f function for Co atom and D95 $(d, p)^{6}$ basis set for $\mathrm{H}, \mathrm{C}, \mathrm{N}$, and $\mathrm{Cl}$ atoms. The open-shell singlet state (OSS) and closed-shell singlet state (CSS) were used for the optimization of TS $\mathrm{TST}_{\mathrm{ET}}$ OSS was computed by using the broken-symmetry approach. Solvent effects are estimated for methanol using the polarization continuum model method (PCM). ${ }^{7}$ The Gaussian 09 program ${ }^{8}$ was used for all the calculations.

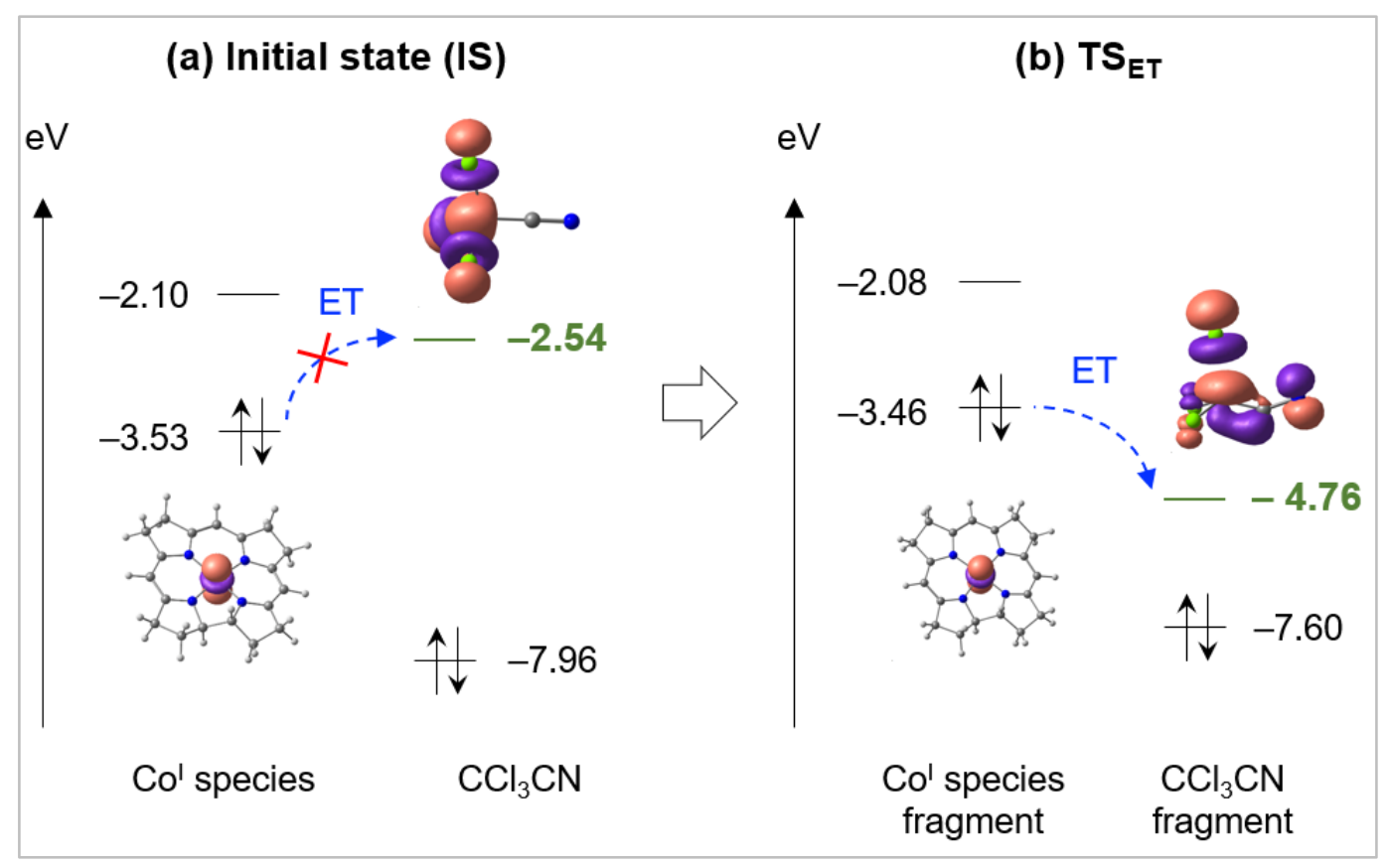

Figure S10. Molecular orbital analysis for reaction of $\mathrm{Co}(\mathrm{I})$ with $\mathrm{CN}-\mathrm{CCl}_{3}$.

Table S1. Cartesian coordinates and SCF energy of Co(I) species at the TPSS level of theory. Units are in $\AA$ and Hartree.

$E=-2338.98127316$

$\begin{array}{rrrr}\text { Co } & -0.038227 & 0.000000 & -0.000001 \\ \text { C } & 2.709400 & -0.663092 & -0.374484 \\ \text { C } & 3.652569 & -1.795154 & 0.068052 \\ \text { C } & 2.817459 & -3.061801 & -0.256326 \\ \text { H } & 3.019647 & -3.905333 & 0.415621 \\ \text { C } & 1.394978 & -2.543073 & -0.120094 \\ \text { C } & 0.218582 & -3.324888 & 0.018942 \\ \text { C } & -1.044374 & -2.765687 & 0.083777\end{array}$



C $\quad-2.312002 \quad-3.577903 \quad 0.293260$
$\begin{array}{llll}\text { C } & -3.429199 & -2.566426 & -0.032671\end{array}$
H $\quad-4.269899 \quad-2.598935 \quad 0.670937$
$\begin{array}{llll}\text { C } & -2.693909 & -1.238779 & 0.010725\end{array}$
$\begin{array}{llll}\text { C } & -3.338107 & -0.000001 & -0.000001\end{array}$
$\begin{array}{llll}\text { H } & -4.427449 & -0.000002 & -0.000002\end{array}$
$\begin{array}{lllll}\text { C } & -2.693910 & 1.238777 & -0.010726\end{array}$
C $\quad-3.429201 \quad 2.566423 \quad 0.032670$
$\begin{array}{llll}\text { C } & -2.312005 & 3.577901 & -0.293258\end{array}$
$\begin{array}{llll}\text { H } & -2.333818 & 4.478267 & 0.331772\end{array}$
$\begin{array}{lllll}\text { C } & -1.044376 & 2.765686 & -0.083775\end{array}$
$\begin{array}{lllll}\text { C } & 0.218579 & 3.324888 & -0.018940\end{array}$
C $\begin{array}{llll}1.394976 & 2.543074 & 0.120095\end{array}$
C $\quad 2.817457 \quad 3.061803 \quad 0.256328$
$\begin{array}{lllll}\text { C } & 3.652568 & 1.795157 & -0.068053\end{array}$
H $\quad 3.828097 \quad 1.729545 \quad-1.151207$
$\begin{array}{llll}\text { C } & 2.709400 & 0.663093 & 0.374481\end{array}$
H $\quad 2.8195540 .460188 \quad 1.453143$
N $\quad \begin{array}{llll}1.340165 & -1.213927 & -0.149669\end{array}$
$\begin{array}{lllll}N & -1.329350 & -1.398874 & 0.014164\end{array}$
$\begin{array}{lllll}N & -1.329351 & 1.398873 & -0.014164\end{array}$
$\begin{array}{llll}N & 1.340164 & 1.213928 & 0.149667\end{array}$
$\begin{array}{lllll}\text { H } & -2.368153 & 3.900035 & -1.343921\end{array}$
$\begin{array}{lllll}H & -4.269901 & 2.598932 & -0.670939\end{array}$
$\begin{array}{llll}H & -3.835626 & 2.732049 & 1.042353\end{array}$
$\begin{array}{llll}H & -2.333814 & -4.478270 & -0.331769\end{array}$
H $\quad-2.368151 \quad-3.900035 \quad 1.343923$
$\begin{array}{llll}H & -3.835622 & -2.732053 & -1.042354\end{array}$
H $\quad 2.998974 \quad-3.406511 \quad-1.287063$
H $3.828100 \quad-1.729540 \quad 1.151206$
H $4.619386 \quad-1.769270 \quad-0.447262$
$\begin{array}{llll}\text { H } & 3.019644 & 3.905337 & -0.415617\end{array}$
H $4.619385 \quad 1.769273 \quad 0.447259$
H $\quad 2.998972 \quad 3.406510 \quad 1.287065$
H $2.819554 \quad-0.460186 \quad-1.453145$
$\begin{array}{llll}H & 0.309273 & 4.407828 & -0.083916\end{array}$
$\begin{array}{lllll}H & 0.309277 & -4.407827 & 0.083921\end{array}$ 
Table S2. Cartesian coordinates and SCF energy of RC at the TPSS level of theory. Units are in $\AA$ and Hartree.

$$
E=-3850.53327392
$$

$$
\begin{aligned}
& \text { Co } \quad-0.049761 \quad-0.155225 \quad-1.089291 \\
& \text { C } \quad-2.781128 \quad-0.652326 \quad-0.458033 \\
& \text { C }-3.790219-1.792403-0.671886 \\
& \text { C } \quad-2.974088 \quad-3.030184 \quad-0.214379 \\
& \begin{array}{llll}
H & -3.252197 & -3.957264 & -0.731247
\end{array} \\
& \text { C }-1.550028-2.597452-0.524243 \\
& \begin{array}{llll}
\text { C } & -0.407735 & -3.435592 & -0.603741
\end{array} \\
& \begin{array}{llll}
\text { C } & 0.863492 & -2.942269 & -0.836533
\end{array} \\
& \begin{array}{llll}
\text { C } & 2.087683 & -3.829762 & -0.993459
\end{array} \\
& \begin{array}{llll}
\text { C } & 3.255848 & -2.826025 & -0.922658
\end{array} \\
& \text { H } 4.030086 \quad-2.999302-1.680142 \\
& \text { C } 2.557569-1.490914-1.109936 \\
& \begin{array}{llll}
\text { C } & 3.238298 & -0.289106 & -1.305421
\end{array} \\
& \begin{array}{llll}
\text { H } & 4.324843 & -0.331469 & -1.369407
\end{array} \\
& \begin{array}{llll}
\text { C } & 2.636491 & 0.967851 & -1.385704
\end{array} \\
& \text { C } 3.413798 \quad 2.256098 \quad-1.579110 \\
& \begin{array}{llll}
\text { C } & 2.352998 & 3.333389 & -1.276949
\end{array} \\
& \text { H } 2.369824 \quad 4.180752 \quad-1.972309 \\
& \begin{array}{llll}
\text { C } & 1.048580 & 2.557315 & -1.347748
\end{array} \\
& \begin{array}{llll}
\text { C } & -0.195672 & 3.157677 & -1.392626
\end{array} \\
& \begin{array}{llll}
\text { C } & -1.404531 & 2.412953 & -1.394221
\end{array} \\
& \begin{array}{llll}
\text { C } & -2.813088 & 2.975826 & -1.486306
\end{array} \\
& \begin{array}{llll}
\text { C } & -3.667310 & 1.782466 & -0.983919
\end{array} \\
& \text { H } \quad-3.772811 \quad 1.832826 \quad 0.109305 \\
& \begin{array}{llll}
\text { C } & -2.796677 & 0.571306 & -1.363357
\end{array} \\
& \begin{array}{llll}
\text { H } & -2.996841 & 0.247975 & -2.398812
\end{array} \\
& \text { N } \quad-1.454779-1.284232-0.708480 \\
& \text { N } \quad \begin{array}{lll}
1.192181 & -1.595451 & -0.999829
\end{array} \\
& \begin{array}{llll}
N & 1.283137 & 1.180172 & -1.315925
\end{array}
\end{aligned}
$$



$\begin{array}{llll}N & -1.397261 & 1.086979 & -1.301025\end{array}$
$\begin{array}{llll}\text { C } & 0.255674 & 0.404602 & 2.820184\end{array}$
Cl $\quad-0.000840 \quad-1.320054 \quad 2.381780$
Cl $\begin{array}{llll}-1.095312 & 1.438790 & 2.218116\end{array}$
H $2.480371 \quad 3.731154 \quad-0.259240$
H $4.290120 \quad 2.309866-0.922009$
H $3.770894 \quad 2.326141 \quad-2.618089$
H $2.130325 \quad-4.611017 \quad-0.225451$
H $2.054204 \quad-4.328490 \quad-1.973604$
H $3.745918 \quad-2.851337 \quad 0.062698$
H $\quad-3.089093 \quad-3.203914 \quad 0.867735$
H $\quad-4.035124 \quad-1.877547 \quad-1.740239$
H $\quad-4.718637 \quad-1.651914 \quad-0.106744$
H $\quad-2.936966 \quad 3.886649 \quad-0.887004$
H $\quad-4.666973 \quad 1.745397 \quad-1.431434$
H $\quad \begin{array}{llll}3.054789 & 3.229021 & -2.531051\end{array}$
H $\quad-2.793555 \quad-0.312240 \quad 0.590898$
H $\quad-0.245848 \quad 4.244845 \quad-1.415446$
H $\quad-0.532513 \quad-4.511193 \quad-0.491119$
Cl $\quad 0.395566 \quad 0.570999 \quad 4.623789$
C $\quad 1.509868 \quad 0.885525 \quad 2.225016$
$\begin{array}{llll}N & 2.535770 & 1.305789 & 1.833990\end{array}$ 
Table S3. Cartesian coordinates and SCF energy of $\mathrm{TS}_{\mathrm{ET}}$ at the TPSS level of theory. Units are in $\AA$ and Hartree.
Imaginary frequency $226.4996 \mathrm{i} \mathrm{cm}{ }^{-1}$
$E=-3850.53116142$
Co $\quad 0.392006-0.006089-0.829027$
C $\quad-2.360805-0.637433-1.324427$
C $\quad-3.126812-1.754880-2.052282$
C $-2.435455-3.032278-1.507660$
$\mathrm{H} \quad-2.428267-3.869733-2.215482$
C $-1.038446-2.526247-1.193230$
C $\quad 0.114266-3.329319 \quad-0.973562$
C $\quad 1.356889-2.796004-0.694429$
C $\quad 2.612177-3.636561 \quad-0.530173$
C $\quad 3.676004-2.606365-0.099393$
H $\quad 4.607172 \quad-2.672974 \quad-0.674572$
C $\quad 2.974009-1.280257 \quad-0.316968$
$\begin{array}{llll}\text { C } & 3.623817 & -0.049499 & -0.217296\end{array}$
H $4.689839-0.063800 \quad 0.002211$
C $\quad 3.012734 \quad 1.199573 \quad-0.349644$
C $3.761544 \quad 2.511623 \quad-0.222769$
C $\quad 2.617387 \quad 3.540619-0.119292$
H $2.776826 \quad 4.445650 \quad-0.715759$
C $\quad 1.407752 \quad 2.752960-0.589105$
C $\quad 0.195982 \quad 3.324874 \quad-0.919872$
C $\quad-0.913844 \quad 2.554805-1.367955$
C $\quad-2.230590 \quad 3.098237 \quad-1.896238$
C $\quad-3.138026 \quad 1.838872-1.873656$
H $\quad-3.644540 \quad 1.758706 \quad-0.902252$
C $\quad-2.118252 \quad 0.695871-2.020603$
H $\quad-1.8906620 .503798 \quad-3.082759$
N $\quad-0.997792-1.205952-1.121237$
N $\quad 1.643161 \quad-1.437053 \quad-0.587342$
N $1.688290 \quad 1.387666 \quad-0.615862$
N $\quad-0.881733 \quad 1.234496 \quad-1.383716$
C $\quad-0.961335 \quad 0.006285 \quad 2.298839$
Cl $-1.643408 \quad-1.614520 \quad 2.014534$ 

Cl $\quad-2.008368 \quad 1.318356 \quad 1.706736$
H $2.460035 \quad 3.8484320 .924593$
H $4.435656 \quad 2.513325 \quad 0.641561$
H $4.371930 \quad 2.676918-1.122929$
H $2.462962 \quad-4.440497 \quad 0.199992$
H $2.869234-4.104382-1.490576$
$\begin{array}{llll}\mathrm{H} & 3.936286 & -2.708666 & 0.963977\end{array}$
$\begin{array}{llll}\mathrm{H} & -2.913792 & -3.380439 & -0.578563\end{array}$
H $\quad-2.964481-1.673831 \quad-3.136301$
H $\quad-4.203596 \quad-1.729062-1.853203$
H $\quad-2.608386 \quad 3.924255-1.281210$
$\begin{array}{llll}H & -3.893128 & 1.837626 & -2.666931\end{array}$
$\begin{array}{llll}H & -2.092310 & 3.480316 & -2.919599\end{array}$
H $\quad-2.794255 \quad-0.446203 \quad-0.330050$
H $0.0982824 .406836 \quad-0.864393$
H $\quad 0.020660 \quad-4.410485-1.050458$
Cl $\quad-1.049088 \quad 0.225310 \quad 4.442692$
C $\quad 0.441872 \quad 0.123662 \quad 1.920209$
$\begin{array}{llll}\mathrm{N} & 1.578053 & 0.232542 & 2.286176\end{array}$ 
Table S4. Cartesian coordinates and SCF energy of TS SN2 $_{\text {at }}$ at the TPSS level of theory. Units are in $\AA$ and Hartree.
Imaginary frequency $206.0311 \mathrm{i} \mathrm{cm} \mathrm{cm}^{-1}$
$E=-3850.52648377$
Co $\quad-0.040634 \quad 0.037280 \quad-0.935844$
C $\quad 2.762944 \quad 0.564609 \quad-0.676207$
C $\quad 3.730284 \quad 1.657380-1.158851$
$\begin{array}{llll}\text { C } & 2.970762 & 2.953340 & -0.771477\end{array}$
H $3.171905 \quad 3.802016-1.436089$
C $\quad 1.5220802 .501144-0.830981$
C $\quad 0.377504 \quad 3.342582 \quad-0.822263$
C $\quad-0.913140 \quad 2.850195 \quad-0.858663$
C $\quad-2.149183 \quad 3.727505-0.787458$
$\begin{array}{llll}\text { C } & -3.281978 & 2.767160 & -1.200353\end{array}$
$\begin{array}{llll}\text { H } & -3.604950 & 2.945483 & -2.236412\end{array}$
C $\quad-2.618595 \quad 1.406437-1.105590$
C $\quad-3.3164620 .205952-1.205358$
H $\quad-4.398592 \quad 0.261155 \quad-1.307394$
C $-2.737286-1.066586-1.156842$
C $\quad-3.542201-2.347747-1.216339$
$\begin{array}{llll}\text { C } & -2.501005 & -3.413370 & -0.820697\end{array}$
H $\quad-2.539621 \quad-4.323355 \quad-1.429567$
C $-1.183542-2.676405-0.972398$
C $0.049265-3.294549-0.975459$
C $\quad \begin{array}{llll}1.266327 & -2.569438 & -1.113962\end{array}$
C $\quad 2.655295-3.165076-1.265801$
C $3.567364-1.939025-0.996014$
H $3.789918 \quad-1.867105 \quad 0.077657$
$\begin{array}{llll}\text { C } & 2.670417 & -0.761869 & -1.417991\end{array}$
H $2.750148 \quad-0.570204-2.501165$
$\begin{array}{llll}\text { N } & 1.415306 & 1.181479 & -0.832027\end{array}$
$\begin{array}{llll}N & -1.258778 & 1.503641 & -0.950713\end{array}$
N $\quad-1.396660 \quad-1.299995 \quad-1.076648$
N $1.286809-1.248581-1.144273$
$\begin{array}{llll}\text { C } & 0.032952 & -0.075160 & 2.267928\end{array}$
Cl $\quad 0.398485 \quad 1.627991 \quad 2.208071$ 

Cl $\quad 1.356852 \quad-1.204245 \quad 2.133338$
$\begin{array}{llll}\mathrm{H} & -2.623794 & -3.703734 & 0.232583\end{array}$
H $\quad-4.410386-2.311553 \quad-0.548650$
H $\quad-3.912106 \quad-2.504169-2.240558$
$\begin{array}{llll}\mathrm{H} & -2.281890 & 4.075771 & 0.247078\end{array}$
H $\quad-2.061917 \quad 4.611410 \quad-1.428939$
H $\quad-4.169741 \quad 2.831952-0.560620$
H $3.212415 \quad 3.266974 \quad 0.256456$
H $3.842847 \quad 1.599319 \quad-2.250502$
H $4.720109 \quad 1.582572 \quad-0.695959$
H $2.822385 \quad-3.999575 \quad-0.573768$
H $4.511592 \quad-1.972804-1.549851$
H $2.785476 \quad-3.549267-2.289466$
$\begin{array}{llll}H & 2.906693 & 0.361407 & 0.397510\end{array}$
H $0.088550 \quad-4.378390 \quad-0.892316$
$\begin{array}{llll}\mathrm{H} & 0.518527 & 4.420365 & -0.778317\end{array}$
Cl $\quad-0.349011 \quad-0.274538 \quad 4.669197$
$\begin{array}{llll}\text { C } & -1.272616 & -0.500539 & 1.894141\end{array}$
N $\quad-2.398553 \quad-0.868060 \quad 1.823600$ 
Table S5. Cartesian coordinates and SCF energy of alkyl-cobalt intermediate at the TPSS level of theory. Units are in $\AA$ and Hartree.

$$
\begin{aligned}
& E=-3390.22562335 \\
& \text { Co } \quad 0.015297 \quad-0.007254 \quad-0.404543 \\
& \begin{array}{llll}
\text { C } & -2.770986 & -0.652140 & -0.272973
\end{array} \\
& \text { C } \quad-3.702033 \quad-1.765523 \quad-0.782349 \\
& \text { C } \quad-2.871985 \quad-3.048387 \quad-0.512489 \\
& \text { H } \quad-3.044946 \quad-3.852340 \quad-1.236678 \\
& \begin{array}{llll}
\text { C } & -1.447339 & -2.532030 & -0.554837
\end{array} \\
& \text { C } \quad-0.268526-3.327407-0.613171 \\
& \text { C } 1.002226-2.793461 \quad-0.614963 \\
& \begin{array}{llll}
\text { C } & 2.268875 & -3.623582 & -0.636051
\end{array} \\
& \begin{array}{llll}
\text { C } & 3.376260 & -2.581102 & -0.886787
\end{array} \\
& \text { H } \quad 3.798231 \quad-2.659466 \quad-1.897947 \\
& \begin{array}{llll}
\text { C } & 2.647315 & -1.262377 & -0.748783
\end{array} \\
& \begin{array}{llll}
\text { C } & 3.292341 & -0.026167 & -0.812695
\end{array} \\
& \text { H } \quad 4.374074 \quad-0.034126 \quad-0.923895 \\
& \begin{array}{llll}
\text { C } & 2.663587 & 1.218501 & -0.751304
\end{array} \\
& \begin{array}{llll}
\text { C } & 3.407746 & 2.532061 & -0.832322
\end{array} \\
& \text { C } \quad 2.335537 \quad 3.556404 \quad-0.411104 \\
& \text { H } 2.316343 \quad 4.460825 \quad-1.027409 \\
& \text { C } \quad 1.0481692 .767275 \quad-0.520961 \\
& \text { C } \quad-0.211353 \quad 3.324264 \quad-0.517158 \\
& \begin{array}{llll}
\text { C } & -1.392194 & 2.546469 & -0.682268
\end{array} \\
& \begin{array}{llll}
\text { C } & -2.800206 & 3.085684 & -0.850099
\end{array} \\
& \begin{array}{llll}
\text { C } & -3.667726 & 1.829487 & -0.571204
\end{array} \\
& \text { H } \quad-3.879494 \quad 1.752918 \quad 0.503470 \\
& \text { C } \quad-2.732324 \quad 0.686643 \quad-0.998010 \\
& \text { H } \quad-2.794582 \quad 0.503613 \quad-2.082775 \\
& \text { N } \quad-1.406699 \quad-1.216928 \quad-0.471939 \\
& \text { N } 1.299386-1.430115 \quad-0.604746 \\
& \text { N } \quad 1.317009 \quad 1.400317 \quad-0.628152 \\
& \begin{array}{llll}
N & -1.367416 & 1.229480 & -0.718092
\end{array} \\
& \text { C } \quad 0.094749 \quad 0.025696 \quad 1.565961 \\
& \text { Cl } \quad-0.239133 \quad-1.604187 \quad 2.343764 \\
& \begin{array}{llll}
\text { Cl } & -1.035625 & 1.241759 & 2.345507
\end{array}
\end{aligned}
$$



H $2.473292 \quad 3.860793 \quad 0.636091$
H $4.2954932 .528982 \quad-0.190943$
H $3.740492 \quad 2.695225 \quad-1.867299$
H $2.388633-4.1154630 .338666$
H $\quad 2.222932-4.405200-1.401850$
H $\quad 4.207973 \quad-2.645317 \quad-0.175778$
H $\quad-3.064792 \quad-3.452390 \quad 0.493235$
H $\quad-3.868111-1.646295 \quad-1.861185$
H $\quad-4.670219-1.767089-0.272588$
H $\quad-2.995471 \quad 3.920730 \quad-0.167474$
H $\quad-4.612977 \quad 1.828109 \quad-1.122776$
H $\quad-2.930549 \quad 3.454044 \quad-1.878852$
H $\quad-2.895691 \quad-0.480005 \quad 0.808614$
H $\quad-0.303454 \quad 4.403984 \quad-0.431923$
H $\quad-0.373605 \quad-4.408917 \quad-0.649930$
C $\quad 1.437329 \quad 0.376994 \quad 2.013755$
$\begin{array}{llll}N & 2.531655 & 0.644492 & 2.359095\end{array}$ 
Table S6. Cartesian coordinates and SCF energy of radical intermediate at the TPSS level of theory. Units are in $\AA$ and Hartree.
$E=-3390.18406128$
Co $\quad 0.025041 \quad 0.162465 \quad-0.801123$
C $\quad 2.822598 \quad 0.479990 \quad-0.244289$
C $3.897695 \quad 1.545541-0.519315$
C $3.172060 \quad 2.857870 \quad-0.116123$
H $\quad 3.488307 \quad 3.735248 \quad-0.692226$
C $\quad \begin{array}{lll}1.714363 & 2.508567 & -0.361124\end{array}$
C $\quad 0.625676 \quad 3.423758 \quad-0.426788$
$\begin{array}{llll}\text { C } & -0.687336 & 3.019099 & -0.559625\end{array}$
$\begin{array}{llll}\text { C } & -1.861319 & 3.977535 & -0.638343\end{array}$
C $\quad-3.086389 \quad 3.047489 \quad-0.527239$
H $\quad-3.860217 \quad 3.246251 \quad-1.277384$
$\begin{array}{llll}\text { C } & -2.484065 & 1.668306 & -0.700151\end{array}$
$\begin{array}{llll}\text { C } & -3.250848 & 0.509722 & -0.837334\end{array}$
$\begin{array}{llll}\text { H } & -4.332696 & 0.624374 & -0.841030\end{array}$
C $\quad-2.743028-0.787957-0.940470$
$\begin{array}{llll}\text { C } & -3.620232 & -2.015578 & -1.063517\end{array}$
$\begin{array}{llll}\text { C } & -2.618880 & -3.174655 & -0.887386\end{array}$
H $\quad-2.719049 \quad-3.962253 \quad-1.642385$
C $-1.268282-2.488565-0.972076$
C $\quad-0.066444 \quad-3.164314 \quad-1.018819$
$\begin{array}{llll}\text { C } & 1.195087 & -2.504267 & -1.053273\end{array}$
C $2.554469-3.174307-1.147443$
$\begin{array}{llll}\text { C } & 3.515775 & -2.030670 & -0.726278\end{array}$
H $\quad 3.663827 \quad-2.050203 \quad 0.361982$
C $\quad 2.728861 \quad-0.766919-1.119497$
H $2.917852 \quad-0.484345 \quad-2.167760$
$\begin{array}{llll}\text { N } & 1.542118 & 1.205828 & -0.481259\end{array}$
$\begin{array}{llll}N & -1.118890 & 1.697847 & -0.666193\end{array}$
N $\quad-1.415026 \quad-1.101764 \quad-0.949980$
$\begin{array}{llll}N & 1.302101 & -1.189814 & -1.009587\end{array}$
$\begin{array}{llll}\text { C } & -0.291229 & -0.569496 & 2.714767\end{array}$
$\begin{array}{llll}\text { Cl } & -0.049148 & 1.128208 & 2.854621\end{array}$
Cl $\quad \begin{array}{llll}1.069930 & -1.620132 & 2.653388\end{array}$ 

$\begin{array}{llll}\text { H } & -2.721291 & -3.641302 & 0.101785\end{array}$
H $\quad-4.418049-2.009842 \quad-0.312826$
H $\quad-4.092592 \quad-2.028383-2.055906$
$\begin{array}{llll}\mathrm{H} & -1.813700 & 4.737141 & 0.149571\end{array}$
H $\quad-1.839622 \quad 4.497783 \quad-1.605913$
$\begin{array}{llll}H & -3.559519 & 3.112784 & 0.462846\end{array}$
H $3.314580 \quad 3.087800 \quad 0.950975$
H $4.137357 \quad 1.563911-1.591191$
H $4.818646 \quad 1.372440 \quad 0.046755$
H $2.618790 \quad-4.063111 \quad-0.508889$
H $4.493098 \quad-2.087763 \quad-1.216095$
H $2.732135 \quad-3.493740 \quad-2.185642$
H $2.834743 \quad 0.167666 \quad 0.812800$
H $\quad-0.085153 \quad-4.251530-1.020889$
H $\quad 0.833543 \quad 4.488766 \quad-0.355331$
$\begin{array}{llll}\text { C } & -1.573254 & -1.076993 & 2.494185\end{array}$
$\begin{array}{llll}N & -2.666309 & -1.510736 & 2.318695\end{array}$ 
Table S7. Cartesian coordinates and SCF energy of $\mathbf{C N C C l}_{3}$ at the TPSS level of theory. Units are in $\AA$ and Hartree.
$E=-1511.53744670$
C $\quad-0.025448 \quad 0.000348 \quad-0.000667$
Cl $\quad 0.529801 \quad 0.169702 \quad 1.705819$
Cl $\quad 0.573251 \quad 1.377762 \quad-0.997302$
Cl $\quad 0.532424 \quad-1.567408-0.692230$
C $\quad-1.4943640 .018630-0.015270$
$\begin{array}{llll}N & -2.669173 & 0.032170 & -0.025894\end{array}$

Table S8. Cartesian coordinates and SCF energy of A at the TPSS level of theory. Units are in $\AA$ and Hartree.

$E=-1051.31770544$
C $\quad 0.053297 \quad-0.000226 \quad-0.000078$
Cl $\quad-0.805950 \quad 1.489659 \quad 0.000015$
Cl $\quad-0.811580 \quad-1.486848 \quad 0.000015$
$\begin{array}{llll}\text { C } & 1.450104 & -0.002637 & -0.000082\end{array}$
N $\quad 2.639658 \quad-0.004372 \quad 0.000063$ 


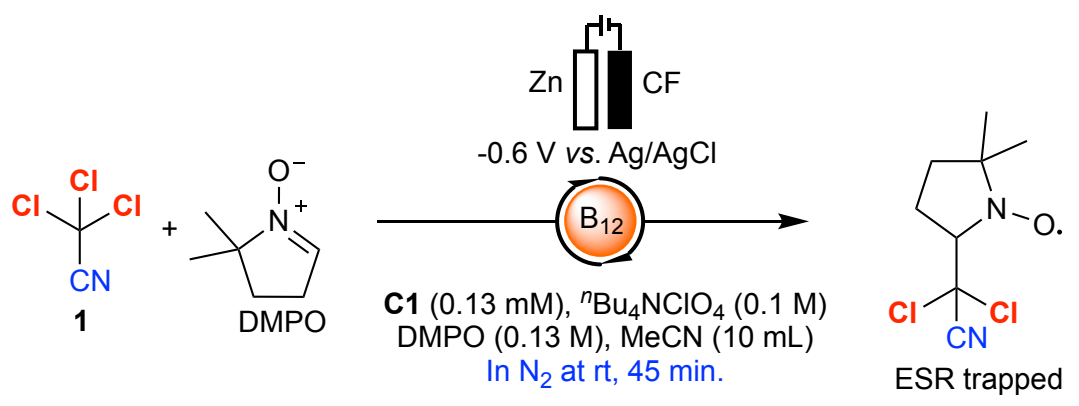

Electrolysis carried out with the standard procedure of bulk electrolysis in presence of the DMPO under continuous bubbling of $\mathrm{N}_{2}$ through the reaction mixture to trap the carboncentered radical produced during the electrolysis. After 45 minutes, a small portion of the solution was drawn by a special-type syringe for the ESR measurement and the ESR was taken at room temperature. The settings for the ESR measurements were as follows: a frequency of $9.78 \mathrm{GHz}$, a power of $1.0 \mathrm{~mW}$, a center field of $3515 \mathrm{G}$, a sweep width of $150 \mathrm{G}$, a modulation amplitude of $5.0 \mathrm{G}$, a time constant of $40 \mathrm{~ms}$, and a sweep time of $20 \mathrm{~s}$.

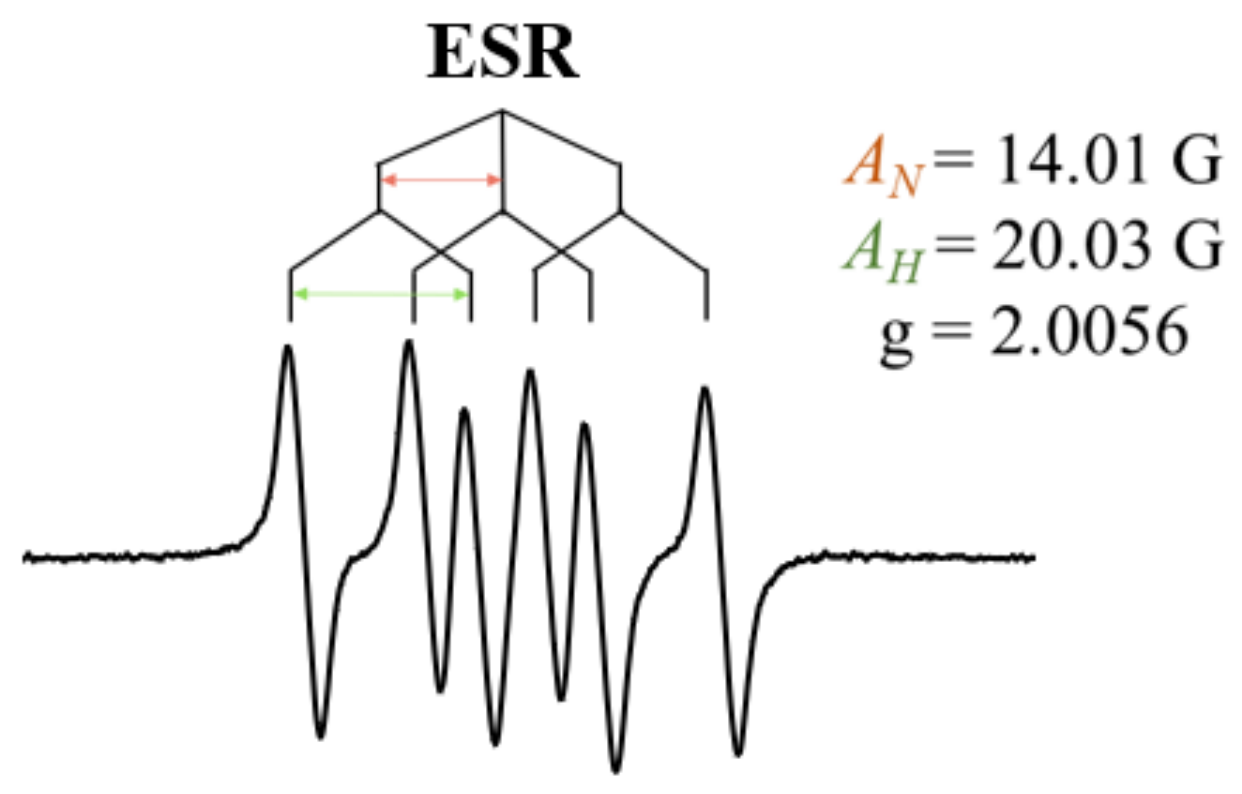

Figure S11. ESR spectrum of DMPO spin adduct (Under $\mathrm{N}_{2}$ ). 


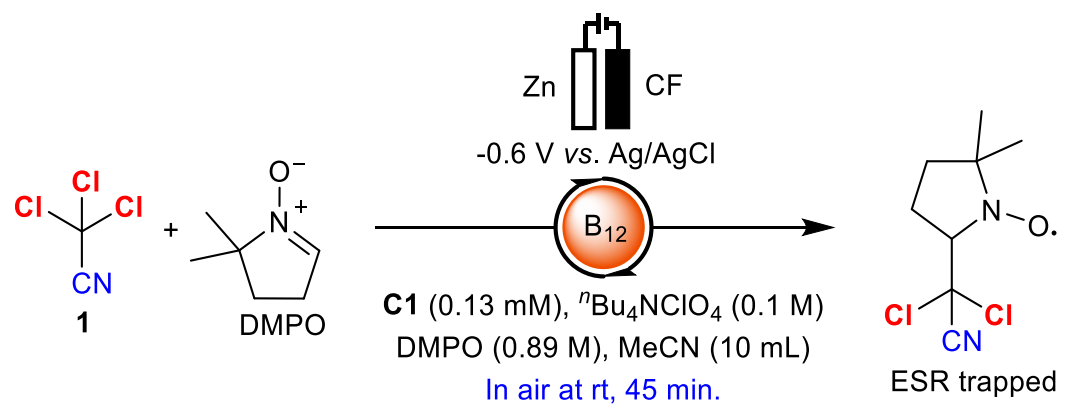

Electrolysis carried out with the standard procedure for bulk electrolysis in presence of the DMPO in air to trap the oxygen-centered radical produced during electrolysis. After 45 minutes, a small portion of the solution was drawn a by special-type syringe for the ESR measurement and the ESR was taken at room temperature. The settings for the ESR measurements were as follows: a frequency of $9.78 \mathrm{GHz}$, a power of $1.0 \mathrm{~mW}$, a center field of $3515 \mathrm{G}$, a sweep width of $150 \mathrm{G}$, a modulation amplitude of $5.0 \mathrm{G}$, a time constant of 40 $\mathrm{ms}$, and a sweep time of $20 \mathrm{~s}$.

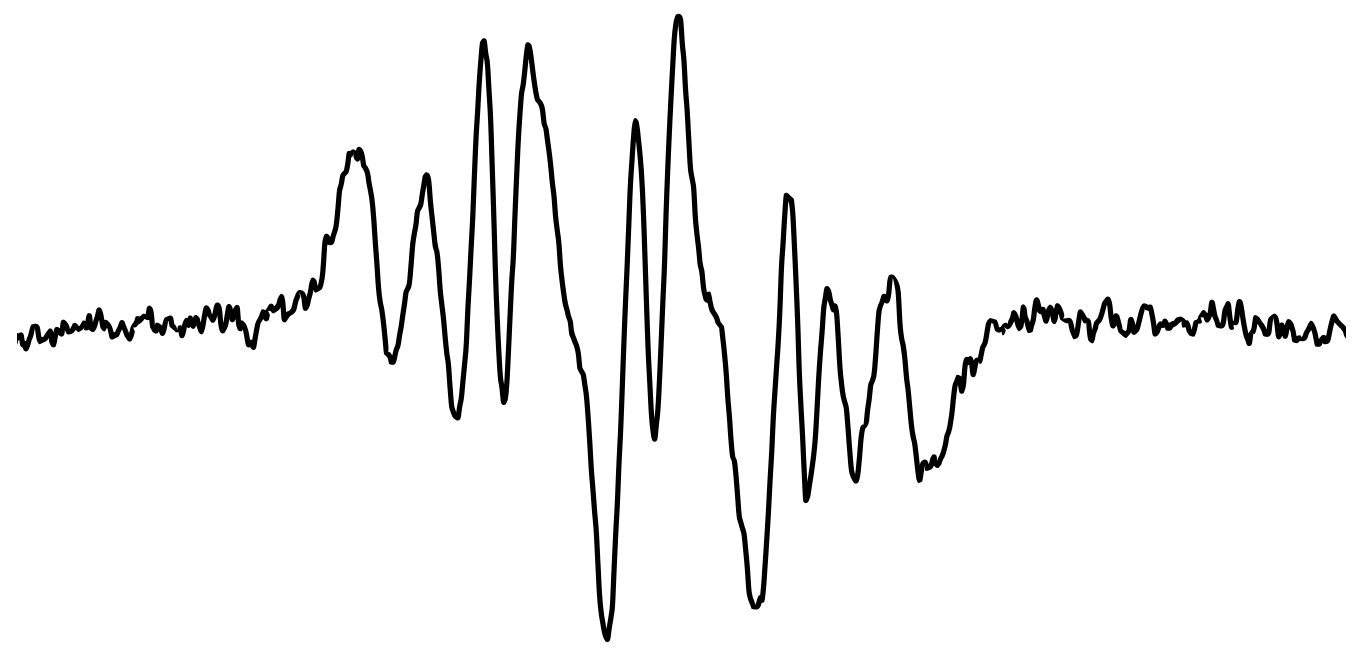

Figure S12. ESR spectrum of DMPO spin adduct (in air). 


\section{f) Source of the oxygen in the products}

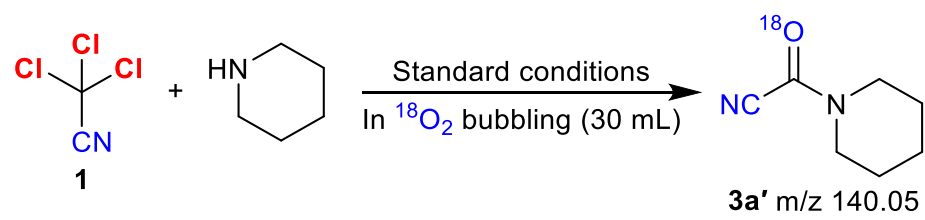

An oven-dried cylinder-type undivided glass cell was equipped with a magnetic stir bar, the catalyst $\mathrm{C} 1(0.13 \mathrm{mM})$, the ${ }^{n} \mathrm{Bu}_{4} \mathrm{NClO}_{4}(0.1 \mathrm{M})$, diphenyl internal standard $(4.2 \mathrm{mM})$, and the substrate $1(20.0 \mathrm{mM})$ dissolved in $10.0 \mathrm{~mL}$ of anhydrous $\mathrm{MeCN}$, then the amine $(0.2 \mathrm{M})$ was added. A CF cathode, a $\mathrm{Zn}$-plate anode, and the $\mathrm{Ag} / \mathrm{AgCl}(3 \mathrm{M}, \mathrm{NaCl})$ reference electrode were placed in the with glass cell and connected to the potentiostat. The reaction mixture was stirred and purged with $\mathrm{N}_{2}$ for 15 minutes to remove atmospheric oxygen then purged with $30 \mathrm{~mL}$ isotopic oxygen $\left({ }^{18} \mathrm{O}_{2}\right)$ gas through the reaction mixture. After electrolysis at -0.6 $\mathrm{V}$ vs. $\mathrm{Ag} / \mathrm{AgCl}$ for $1.5 \mathrm{~h}$, a small portion of the electrolyte solution was passed through a short column packed with silica gel and the reaction mixture was collected using an ethyl acetate eluent, then analyzed by GC-MS and the product 3a' identified as the major product containing isotopic oxygen with the $\mathrm{m} / \mathrm{z} 140.05$ detected by the GC-MS.

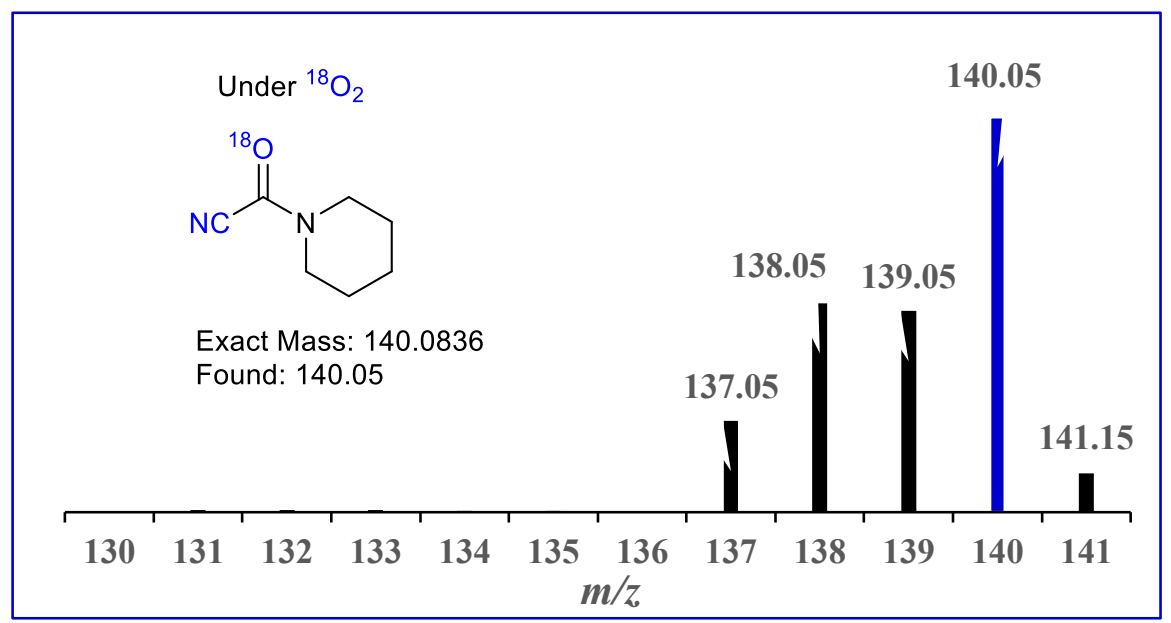

Figure S13. GC-MS spectrum of the product 3a'. 


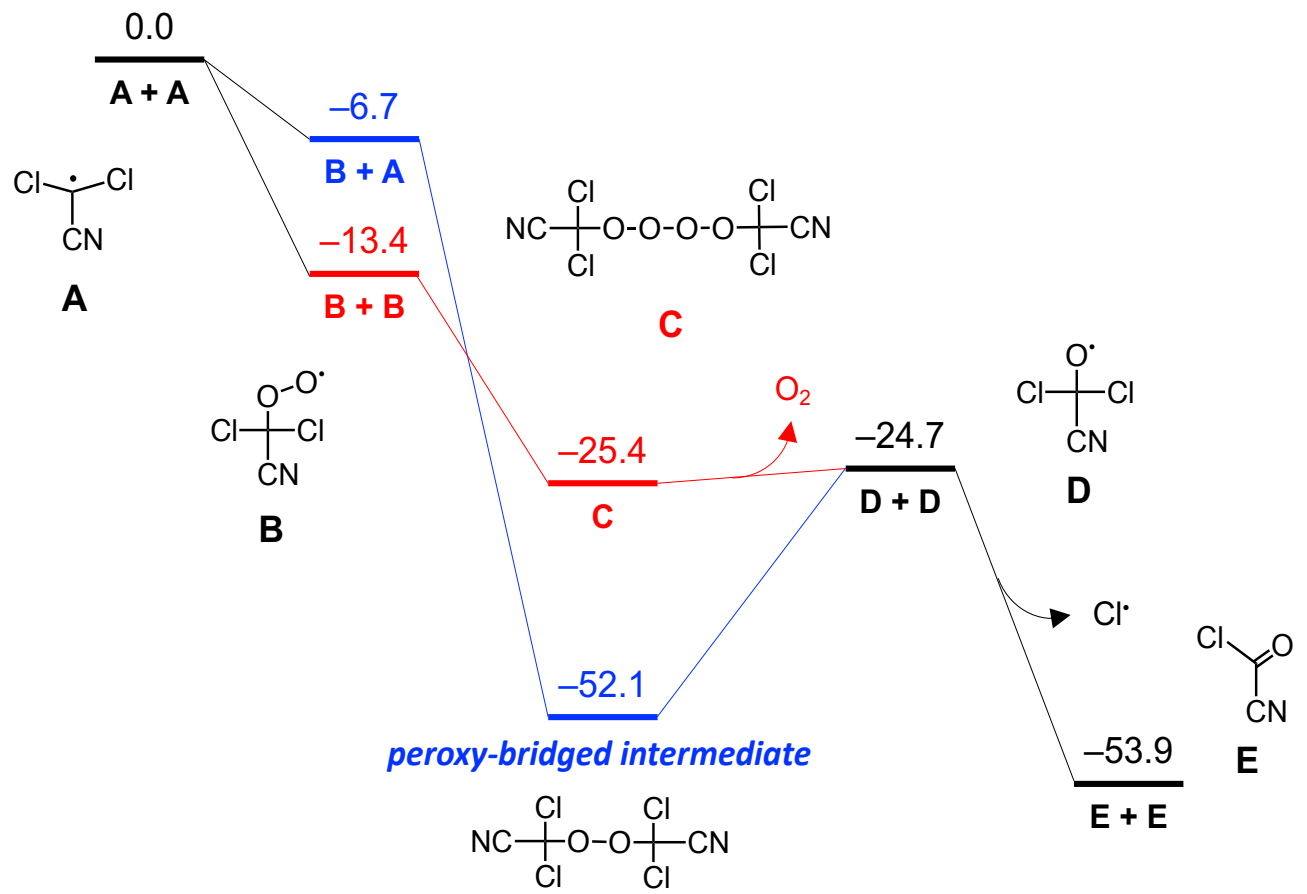

Figure S14 Computed energy diagrams for the cyanoformyl chloride (E) formation steps. Units are in kilocalories per mole.

Table S9. Optimized structure and calculated energy of A.

SCF energy -1051.27888127

Atom Coordinates (Angstroms)

$\mathrm{X}$ Y

Z
C $\quad 0.000988 \quad 0.059994 \quad-0.000000$
C $\quad-0.000949 \quad 1.449453-0.000000$
Cl $\quad 0.000988-0.806299 \quad 1.477945$
Cl $\quad 0.000988 \quad-0.806299 \quad-1.477945$
$\begin{array}{llll}N & -0.004834 & 2.622497 & -0.000000\end{array}$ 
Table S10. Optimized structure and calculated energy of B.

SCF energy -1051.27888127

Atom Coordinates (Angstroms)

$\begin{array}{lll}X & Y & Z\end{array}$

C $\quad 0.001876 \quad 0.153607 \quad 0.000000$

C $\quad-0.339670 \quad 1.577816 \quad 0.000000$

$\begin{array}{llll}\text { O } & 1.464103 & 0.129781 & -0.000000\end{array}$

Cl $\quad-0.615785-0.634912 \quad 1.475758$

Cl $\quad-0.615785-0.634912-1.475758$

$\begin{array}{llll}N & -0.615785 & 2.703491 & 0.000000\end{array}$

O $1.945138-1.095526-0.000000$

Table S11. Optimized structure and calculated energy of C.

SCF energy -2403.20861530

Atom Coordinates (Angstroms)

$\begin{array}{lll}X & Y & Z\end{array}$

C $\quad-0.877051 \quad-2.695334 \quad 0.000000$

C $\quad-2.238133 \quad-3.245701 \quad 0.000000$

$\begin{array}{llll}\text { O } & -1.080880 & -1.322177 & 0.000000\end{array}$

$\begin{array}{llll}\text { Cl } & -0.000018 & -3.257144 & 1.478081\end{array}$

Cl $\quad-0.000018-3.257144 \quad-1.478081$

$\begin{array}{llll}N & -3.303432 & -3.702058 & 0.000000\end{array}$

$\begin{array}{llll}\text { O } & 0.228839 & -0.691245 & -0.000000\end{array}$

$\begin{array}{llll}\text { O } & -0.228925 & 0.691346 & 0.000000\end{array}$

$\begin{array}{llll}\text { O } & 1.080807 & 1.322278 & -0.000000\end{array}$ 

C $\quad 0.877004 \quad 2.695417 \quad-0.000000$
$\begin{array}{llll}\text { C } & 2.238129 & 3.245681 & -0.000000\end{array}$
Cl $\quad-0.000018 \quad 3.257280 \quad-1.478082$
Cl $\quad-0.000018 \quad 3.257280 \quad 1.478082$
$\begin{array}{llll}\text { N } & 3.303828 & 3.701112 & -0.000000\end{array}$

Table S12. Optimized structure and calculated energy of D.

SCF energy -1126.45390193
Atom Coordinates (Angstroms)
$x$
Y
Z
C $\quad 0.097828 \quad 0.301058-0.000000$
C $\quad-1.363412 \quad 0.484791-0.000000$
$\begin{array}{llll}\text { O } & 0.599636 & 1.541211 & -0.000000\end{array}$
Cl $\quad 0.599636 \quad-0.634360 \quad 1.483171$
Cl $0.599636 \quad-0.634360 \quad-1.483171$
$\begin{array}{llll}N & -2.513031 & 0.646205 & -0.000000\end{array}$

Table S13. Optimized structure and calculated energy of E.

SCF energy -666.344683307

Atom Coordinates (Angstroms)

$X \quad Y \quad Z$
$\begin{array}{llll}\text { C } & 0.002634 & 0.504491 & 0.000345\end{array}$
C $\quad \begin{array}{lll}1.287810 & -0.184267 & 0.000905\end{array}$
O $\quad-0.114775 \quad 1.691804 \quad-0.000402$ 
Cl $\quad-1.360015-0.621251 \quad 0.000055$

N $\quad 2.327970-0.699214 \quad-0.000745$

Table S14. Optimized structure and calculated energy of peroxyl-bridged intermediate.

SCF energy -2252.96036278

Atom Coordinates (Angstroms)

$\mathrm{X}$

Y

Z

C $\quad-0.752642 \quad-1.583180 \quad 0.000000$

C $\quad-2.193383 \quad-1.870558 \quad 0.000000$

O $\quad-0.704224-0.192791 \quad 0.000000$

Cl $\quad 0.000001 \quad-2.295012 \quad 1.477581$

Cl $\quad 0.000001 \quad-2.295012-1.477581$

$\begin{array}{llll}N & -3.325420 & -2.118841 & 0.000000\end{array}$

$\begin{array}{llll}\text { O } & 0.704233 & 0.192777 & 0.000000\end{array}$

$\begin{array}{llll}\text { C } & 0.752649 & 1.583137 & 0.000000\end{array}$

C $\quad 2.193397 \quad 1.870528 \quad 0.000000$

Cl $\quad 0.000001 \quad 2.294984 \quad-1.477582$

$\begin{array}{llll}\text { Cl } & 0.000001 & 2.294984 & 1.477582\end{array}$

$\begin{array}{llll}N & 3.325384 & 2.119057 & 0.000000\end{array}$ 


\section{X-ray crystallography}

Single crystals suitable for X-ray structural analysis were obtained by recrystallization using the solvent diffusion method. The crystals were mounted on a loop. Data from the X-ray diffraction were collected at $123 \mathrm{~K}$ using a Rigaku XtaLAB mini CCD diffractometer equipped with graphite monochromated Mo-Ka radiation $(\lambda=0.71073 \AA)$. The collected data were integrated, corrected, and scaled using the program CrysAlisPro. ${ }^{9}$ The structures were refined using SHELXT (Sheldrick, 2015) ${ }^{10}$ Intrinsic Phasing and SHELXL (Sheldrick, 2015). ${ }^{11}$ All non-hydrogen atoms were anisotropically refined. Hydrogen atoms were located at the calculated positions and included in the structure factor calculation but were not refined. The program Olex2 was used as a graphical interface. ${ }^{12}$ The crystallographic data of the $\mathbf{2 h}, \mathbf{3 c}$, and $\mathbf{3 f}$ complexes are listed in Tables S15-S17, and their CCDC numbers are 2033642, 2033643 , and 2033644 , respectively. 


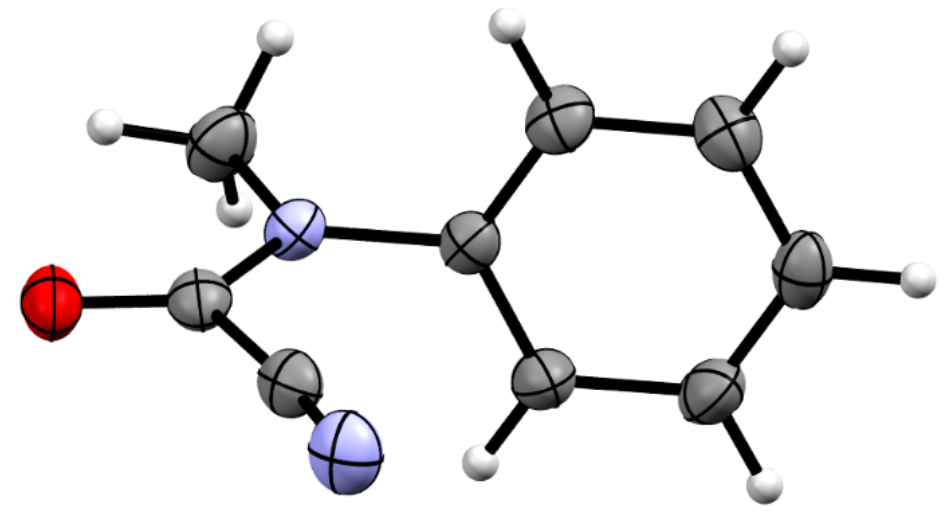

Figure S15. Crystal structure of $\mathbf{2 h}$. Ellipsoids are plotted at the $50 \%$ probability level.

Table S15 Crystallographic data for $\mathbf{2 h}$.

\begin{tabular}{|c|c|}
\hline Compound & $2 \mathrm{~h}$ \\
\hline CCDC No. & 2033642 \\
\hline empirical formula & $\mathrm{C}_{9} \mathrm{H}_{8} \mathrm{~N}_{2} \mathrm{O}$ \\
\hline formula weight & 160.17 \\
\hline temperature $[\mathrm{K}]$ & 123 \\
\hline wavelength $[\AA]$ & 0.71073 \\
\hline crystal system & orthorhombic \\
\hline space group & Pbca \\
\hline$a[\AA]$ & $9.735(2)$ \\
\hline$b[\AA]$ & $7.4750(17)$ \\
\hline$c[\AA]$ & $22.867(5)$ \\
\hline$\alpha\left[^{\circ}\right]$ & 90 \\
\hline$\beta\left[^{\circ}\right]$ & 90 \\
\hline$\gamma\left[{ }^{\circ}\right]$ & 90 \\
\hline Volume $\left[\AA^{3}\right]$ & $1664.1(6)$ \\
\hline$Z$ & 8 \\
\hline Density (calculated) $\left[\mathrm{g} / \mathrm{cm}^{3}\right]$ & 1.279 \\
\hline Absorption coefficient $\left[\mathrm{mm}^{-1}\right]$ & 0.087 \\
\hline$F(000)$ & 672.0 \\
\hline Reflections collected & 4249 \\
\hline Independent reflections & $1702\left[R_{\text {(int) }}=0.0506\right]$ \\
\hline Data / restraints / parameters & $1702 / 0 / 110$ \\
\hline Goodness-of-fit on $F^{2}$ & 1.014 \\
\hline$R 1^{\mathrm{a}}[/>2 \sigma(I)]$ & 0.0588 \\
\hline$w R 2^{\mathrm{b}}$ (all data) & 0.1646 \\
\hline Largest diff. peak and hole $\left[\mathrm{e} . \AA^{-3}\right]$ & 0.29 and -0.26 \\
\hline
\end{tabular}

a: $R 1=\left(\sum|| F_{\mathrm{o}}|-| F_{\mathrm{c}}||\right) /\left(\sum\left|F_{\mathrm{o}}\right|\right)$. b: $w R 2=\left[\sum w\left({F_{\mathrm{o}}}^{2}-{F_{\mathrm{c}}}^{2}\right)^{2} / \sum w F_{\mathrm{o}}^{4}\right]^{1 / 2}$, where $w=$ $1 /\left[\sigma^{2}\left(F_{o}^{2}\right)+\left(0.0790\left(F_{o}^{2}+2 F_{c}^{2}\right) / 3\right)^{2}\right]$ 


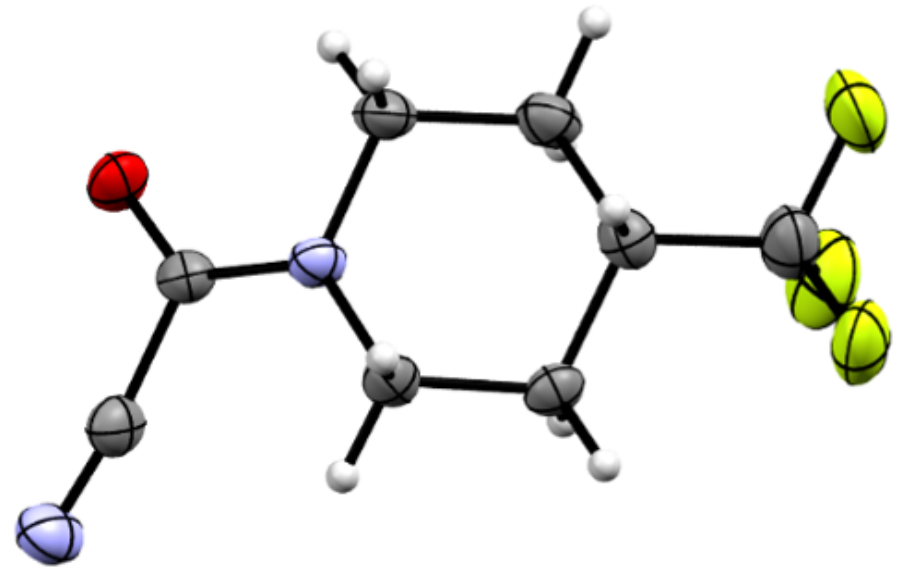

Figure S16. Crystal structure of 3c. Ellipsoids are plotted at the $50 \%$ probability level.

Table S16 Crystallographic data for 3c.

\begin{tabular}{|c|c|}
\hline Compound & $3 c$ \\
\hline CCDC No. & 2033643 \\
\hline empirical formula & $\mathrm{C}_{16} \mathrm{H}_{18} \mathrm{~F}_{6} \mathrm{~N}_{4} \mathrm{O}_{2}$ \\
\hline formula weight & 412.34 \\
\hline temperature $[\mathrm{K}]$ & 123 \\
\hline wavelength $[\AA]]$ & 0.71073 \\
\hline crystal system & monoclinic \\
\hline space group & $\mathrm{Pc}$ \\
\hline$a[\AA]$ & $10.7296(7)$ \\
\hline$b[\AA]$ & $6.4679(3)$ \\
\hline$c[\AA]$ & 14.1827(9) \\
\hline$\alpha\left[^{\circ}\right]$ & 90 \\
\hline$\beta\left[^{\circ}\right]$ & 109.676(7) \\
\hline$\gamma\left[{ }^{\circ}\right]$ & 90 \\
\hline Volume $\left[\AA^{3}\right]$ & $926.78(10)$ \\
\hline$Z$ & 2 \\
\hline Density (calculated) $\left[\mathrm{g} / \mathrm{cm}^{3}\right]$ & 1.478 \\
\hline Absorption coefficient $\left[\mathrm{mm}^{-1}\right]$ & 0.140 \\
\hline$F(000)$ & 424.0 \\
\hline Reflections collected & 10813 \\
\hline Independent reflections & $3756\left[R_{\text {(int) }}=0.0506\right]$ \\
\hline Data / restraints / parameters & 3756 / 2 / 253 \\
\hline Goodness-of-fit on $F^{2}$ & 1.021 \\
\hline$R 1^{a}[I>2 \sigma(I)]$ & 0.0324 \\
\hline$w R 2^{\mathrm{b}}$ (all data) & 0.0828 \\
\hline Largest diff. peak and hole $\left[\mathrm{e} . \AA^{-3}\right]$ & 0.17 and -0.15 \\
\hline
\end{tabular}




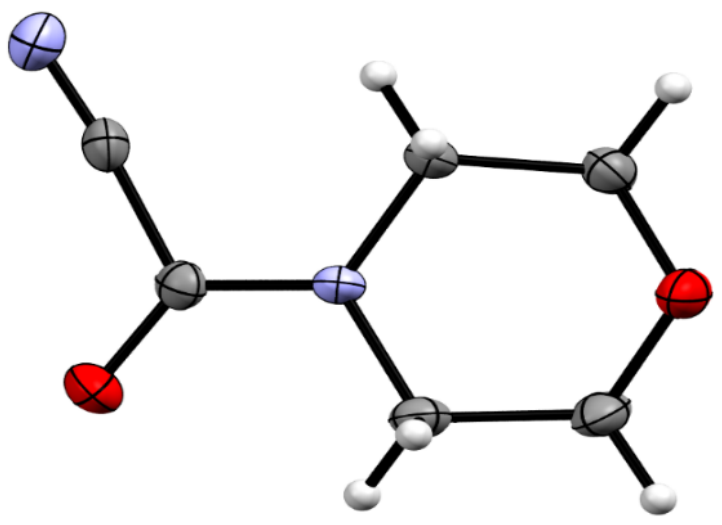

Figure S17. Crystal structure of $\mathbf{3 f}$. Ellipsoids are plotted at the $50 \%$ probability level.

Table S17 Crystallographic data for 3f.

\begin{tabular}{|c|c|}
\hline Compound & $3 f$ \\
\hline CCDC No. & 2033644 \\
\hline empirical formula & $\mathrm{C}_{6} \mathrm{H}_{8} \mathrm{~N}_{2} \mathrm{O}_{2}$ \\
\hline formula weight & 140.14 \\
\hline temperature $[\mathrm{K}]$ & 123 \\
\hline wavelength $[\AA]]$ & 0.71073 \\
\hline crystal system & monoclinic \\
\hline space group & $\mathrm{P} 2{ }_{1} / \mathrm{C}$ \\
\hline$a[\AA]$ & $11.5654(11)$ \\
\hline$b[\AA]]$ & $6.8326(6)$ \\
\hline$c[\AA]$ & $8.7695(8)$ \\
\hline$\alpha\left[^{\circ}\right]$ & 90 \\
\hline$\beta\left[^{\circ}\right]$ & $104.559(9)$ \\
\hline$\gamma\left[^{\circ}\right]$ & 90 \\
\hline Volume $\left[\AA^{3}\right]$ & $670.73(11)$ \\
\hline$Z$ & 4 \\
\hline Density (calculated) $\left[\mathrm{g} / \mathrm{cm}^{3}\right]$ & 1.388 \\
\hline Absorption coefficient $\left[\mathrm{mm}^{-1}\right]$ & 0.106 \\
\hline$F(000)$ & 296.0 \\
\hline Reflections collected & 3977 \\
\hline Independent reflections & $3977\left[R_{\text {(int) }}=\right.$ ?] \\
\hline Data / restraints / parameters & 3977 / 0 / 92 \\
\hline Goodness-of-fit on $F^{2}$ & 0.914 \\
\hline$R 1^{\mathrm{a}}[1>2 \sigma(I)]$ & 0.0367 \\
\hline$w R 2^{\mathrm{b}}$ (all data) & 0.0821 \\
\hline Largest diff. peak and hole $\left[\mathrm{e} . \AA^{-3}\right]$ & 0.27 and -0.25 \\
\hline
\end{tabular}

a: $R 1=\left(\sum|| F_{\mathrm{o}}|-| F_{\mathrm{c}}||\right) /\left(\sum\left|F_{\mathrm{o}}\right|\right) . \mathrm{b}: w R 2=\left[\sum w\left({F_{\mathrm{o}}}^{2}-{F_{\mathrm{c}}}^{2}\right)^{2} / \sum w F_{\mathrm{o}}^{4}\right]^{1 / 2}$, where $w=$ $1 /\left[\sigma^{2}\left(F_{o}^{2}\right)+\left(0.0437\left(F_{o}^{2}+2 F_{c}^{2}\right) / 3\right)^{2}\right]$ 


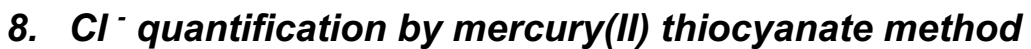

The chloride ions removed from the 1 after electrolysis under aerobic conditions were quantified by the mercury(II) thiocyanate method. ${ }^{13}$ After the electrolysis, $20 \mu \mathrm{L}$ of the solution was added to $1.0 \mathrm{~mL}$ of tetrachloromethane, then $1 \mathrm{~mL}$ of water was added to extract the chloride ions. The procedure was repeated 3 times and the chloride ions in the aqueous solution were quantified by UV-vis spectroscopy.
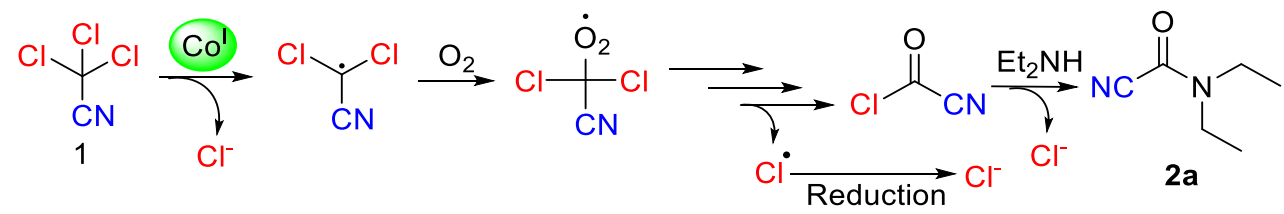

3 equiv. $\mathrm{Cl}^{-}$formed after electrolysis

$$
\begin{gathered}
2 \mathrm{Cl}^{-}+\mathrm{Hg}(\mathrm{SCN})_{2} \rightleftharpoons \mathrm{HgCl}_{2}+2 \mathrm{SCN}^{-} \\
\mathrm{SCN}^{-}+\mathrm{Fe}^{3+} \rightleftharpoons \mathrm{Fe}(\mathrm{SCN})^{2+}
\end{gathered}
$$

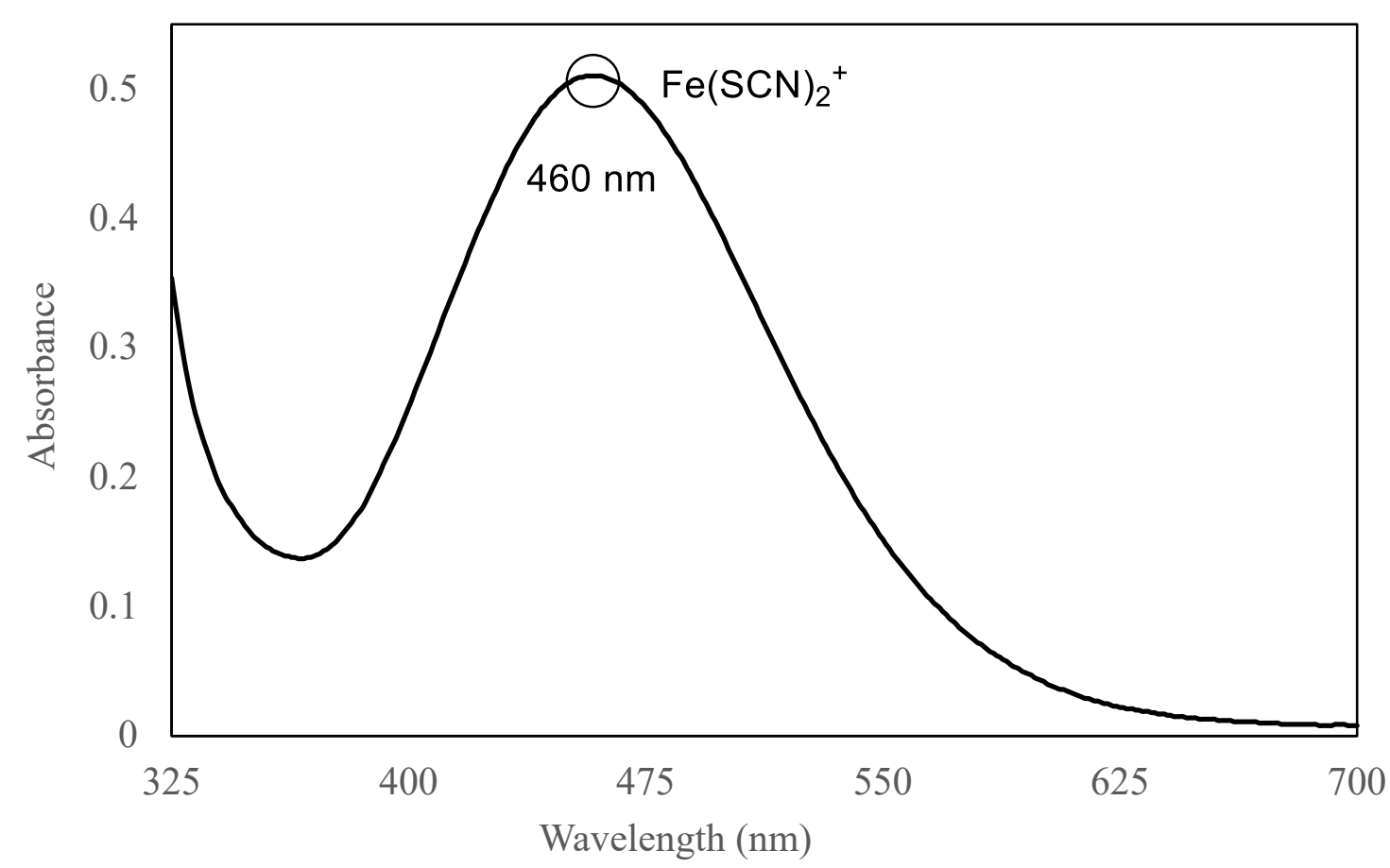

Figure S18. UV-vis absorption spectrum of mercury(II) thiocyanate reagent with solution after the reaction. 


\section{9. ${ }^{1} \mathrm{H}$ and ${ }^{13} \mathrm{C}$ NMR spectra}

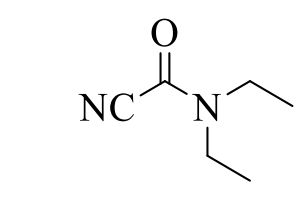

2a

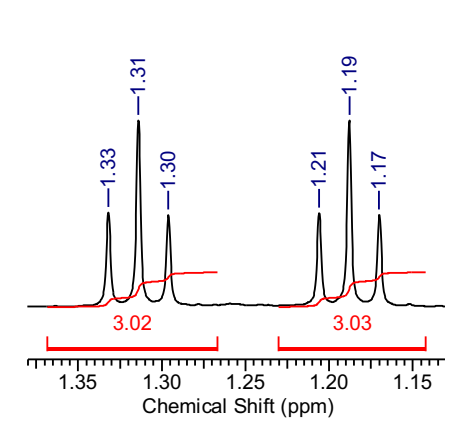

CHLOROFORM-d

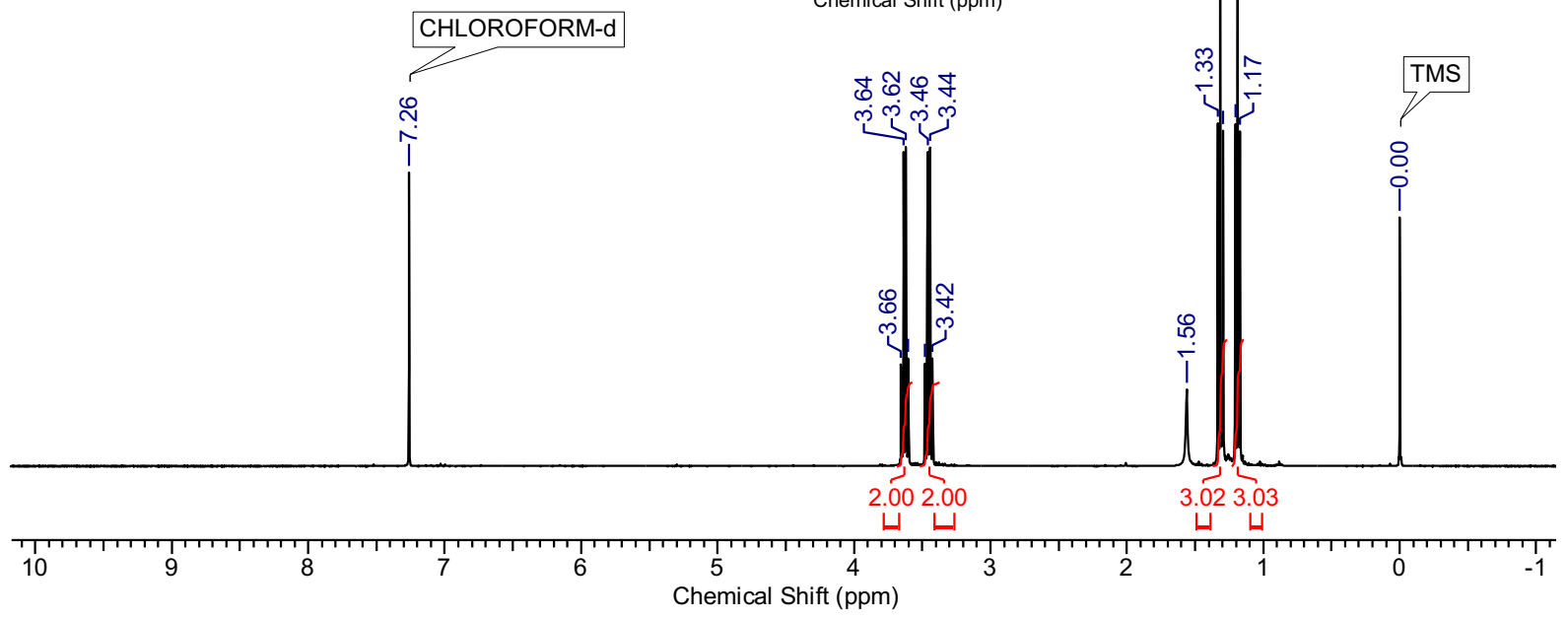

Figure S19. ${ }^{1} \mathrm{H}$ NMR spectrum of $2 \mathrm{a}$ in $\mathrm{CDCl}_{3}(400 \mathrm{MHz})$.

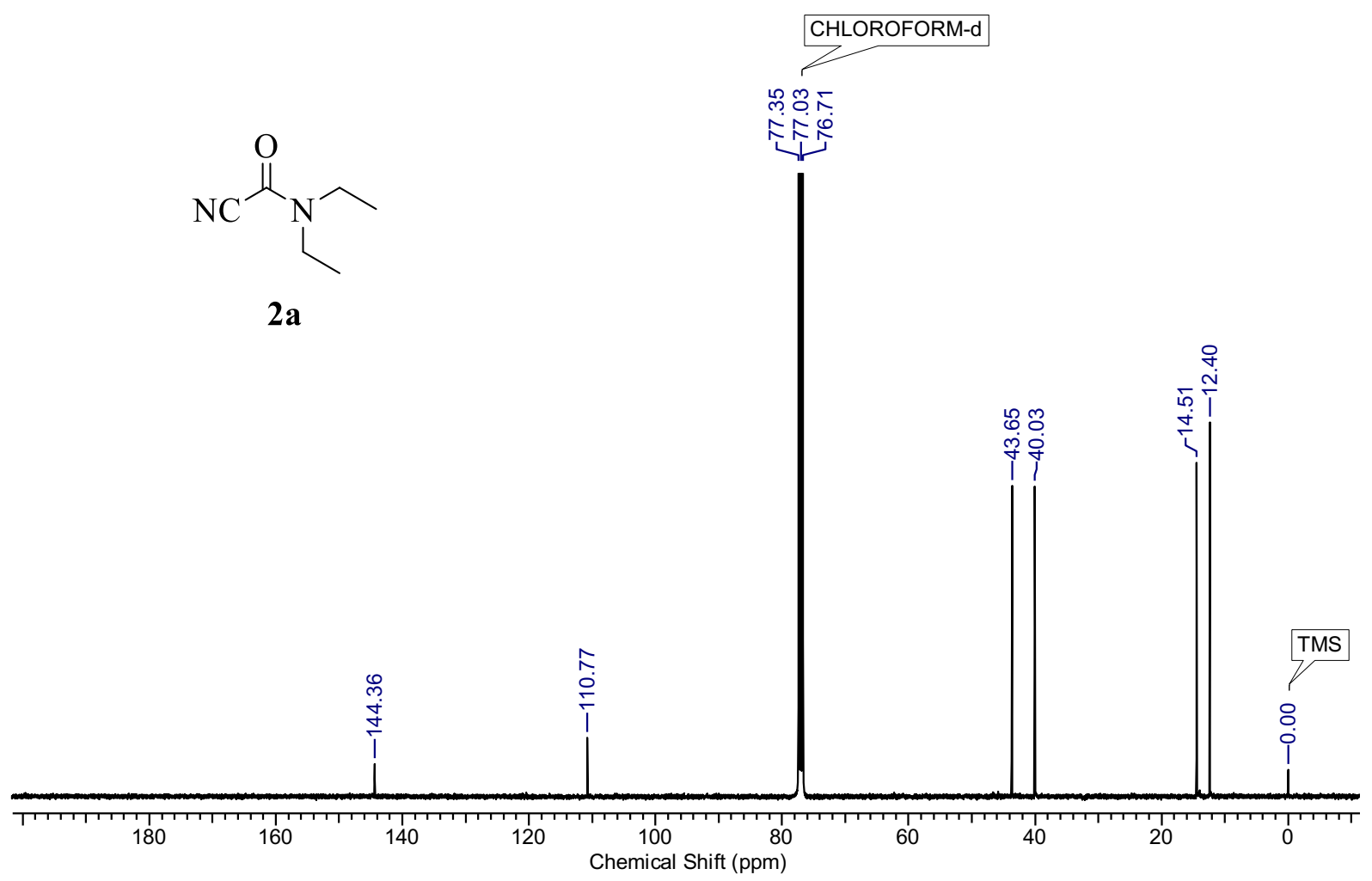

Figure S20. ${ }^{13} \mathrm{C}\left\{{ }^{1} \mathrm{H}\right\}$ NMR spectrum of $\mathbf{2 a}$ in $\mathrm{CDCl}_{3}(100 \mathrm{MHz})$. 


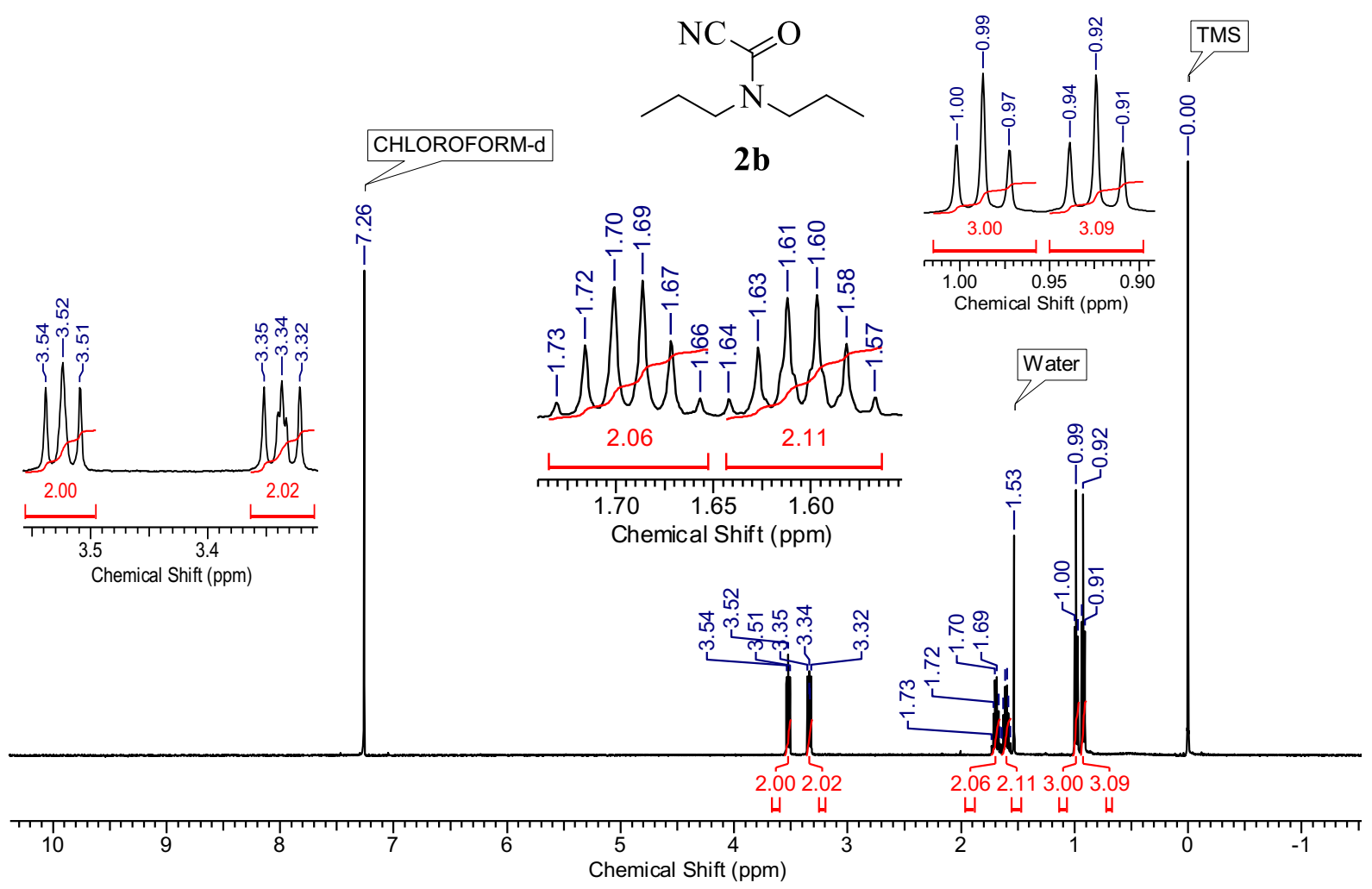

Figure S21. ${ }^{1} \mathrm{H}$ NMR spectrum of $\mathbf{2 b}$ in $\mathrm{CDCl}_{3}(500 \mathrm{MHz})$.

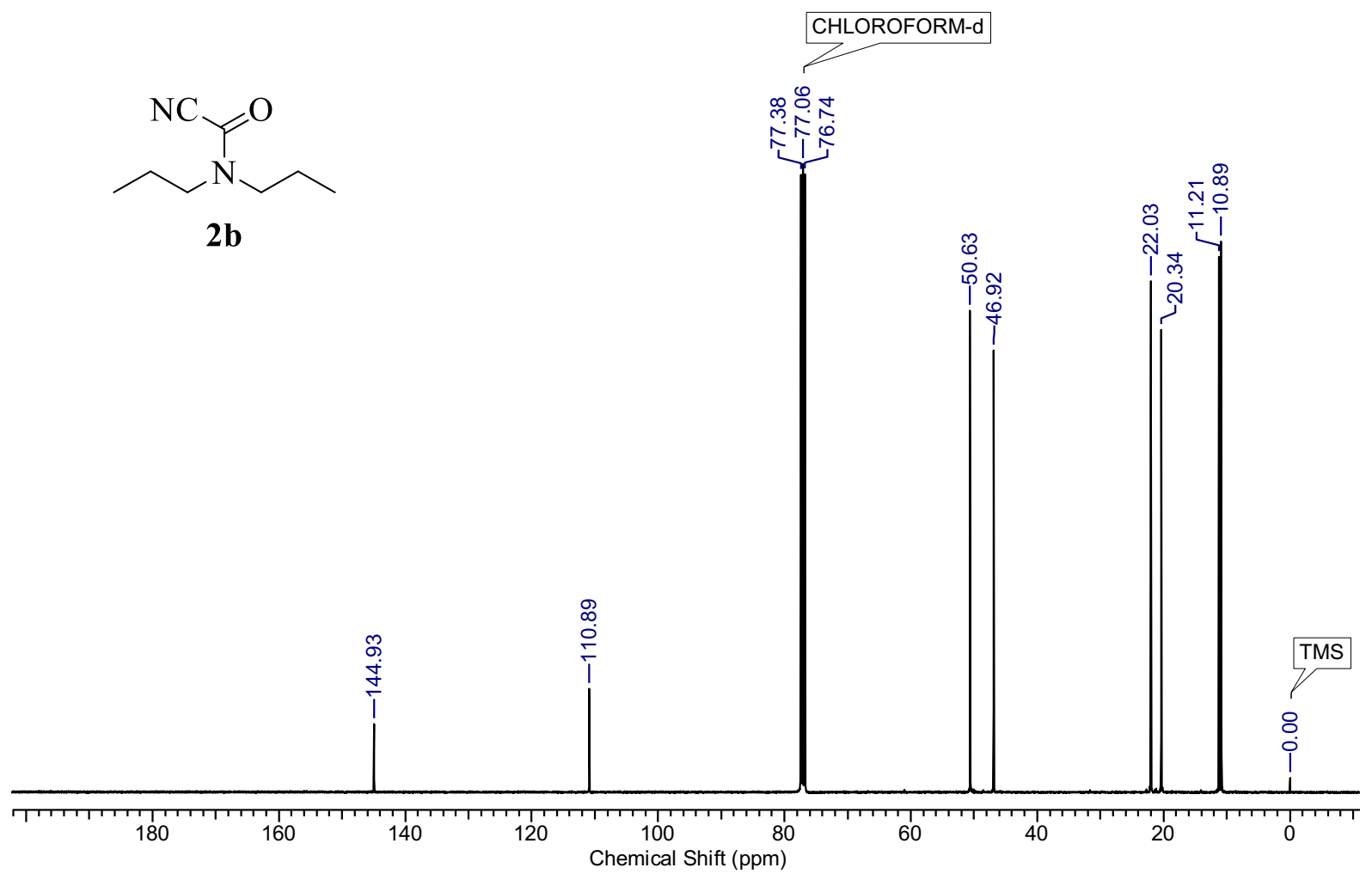

Figure S22. ${ }^{13} \mathrm{C}\left\{{ }^{1} \mathrm{H}\right\}$ NMR spectrum of $\mathbf{2} \mathbf{b}$ in $\mathrm{CDCl}_{3}(100 \mathrm{MHz})$. 


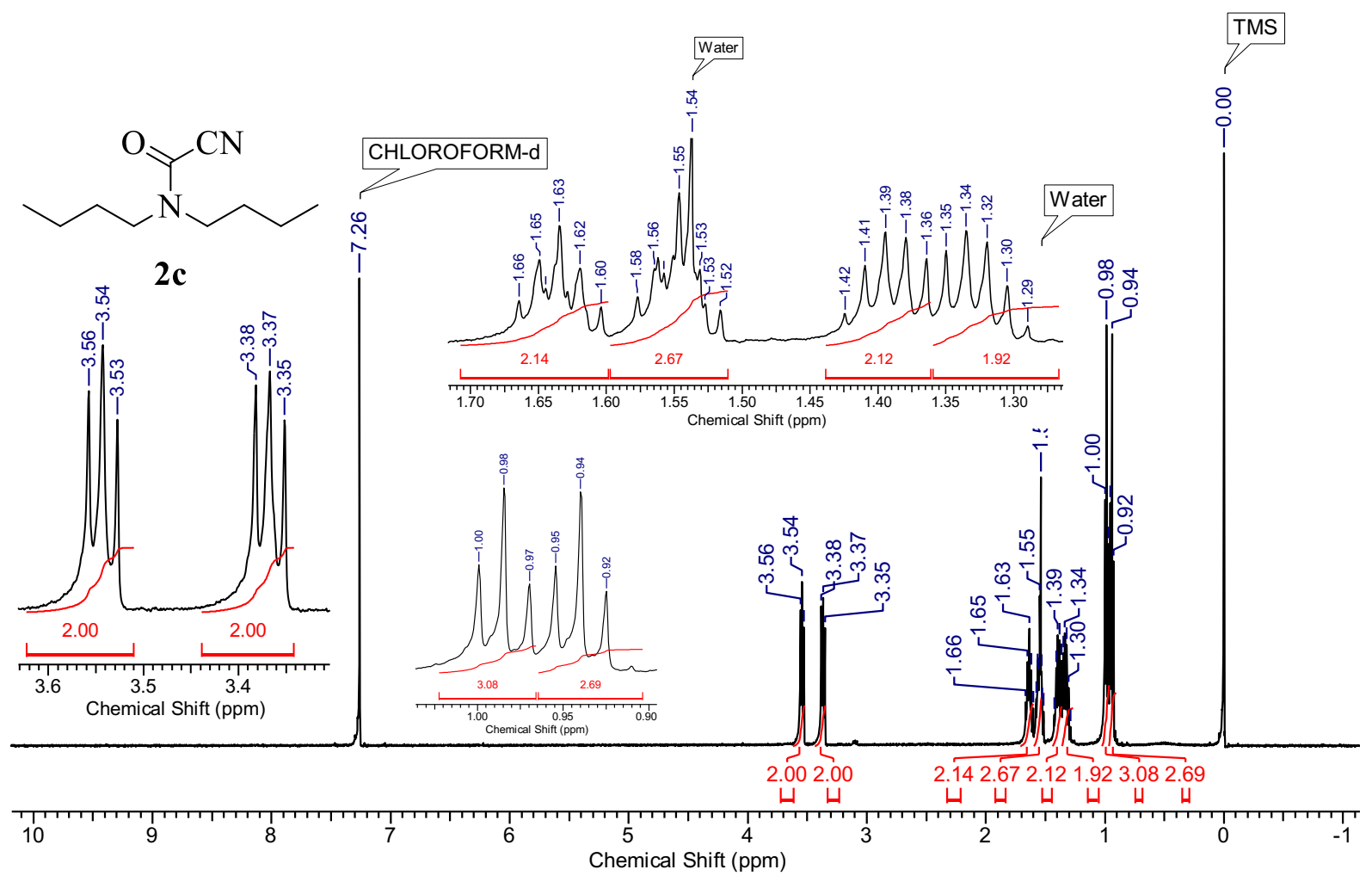

Figure S23. ${ }^{1} \mathrm{H}$ NMR spectrum of $2 \mathrm{c}$ in $\mathrm{CDCl}_{3}(500 \mathrm{MHz})$.

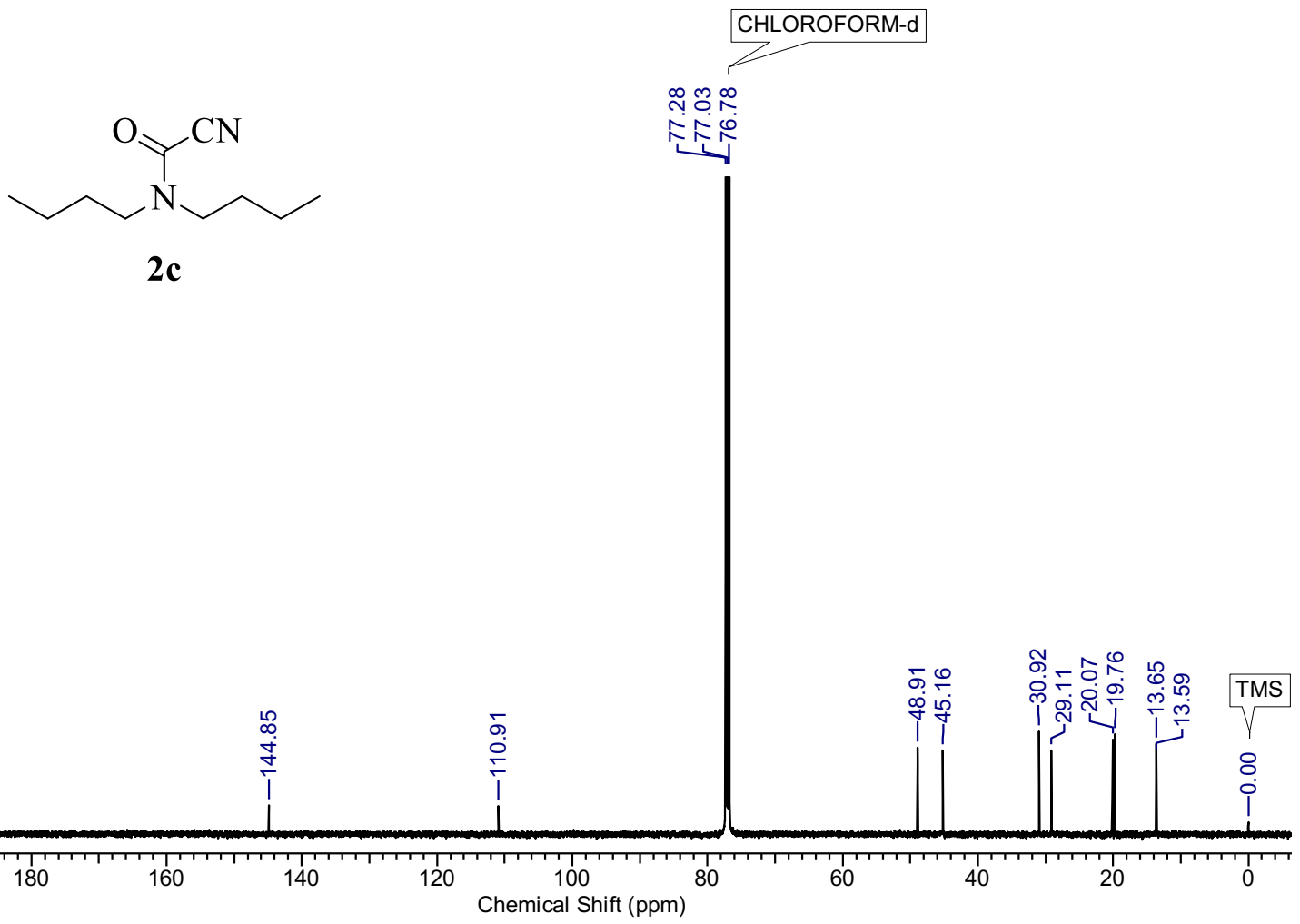

Figure S24. ${ }^{13} \mathrm{C}\left\{{ }^{1} \mathrm{H}\right\}$ NMR spectrum of $2 \mathrm{c}$ in $\mathrm{CDCl}_{3}(125 \mathrm{MHz})$. 


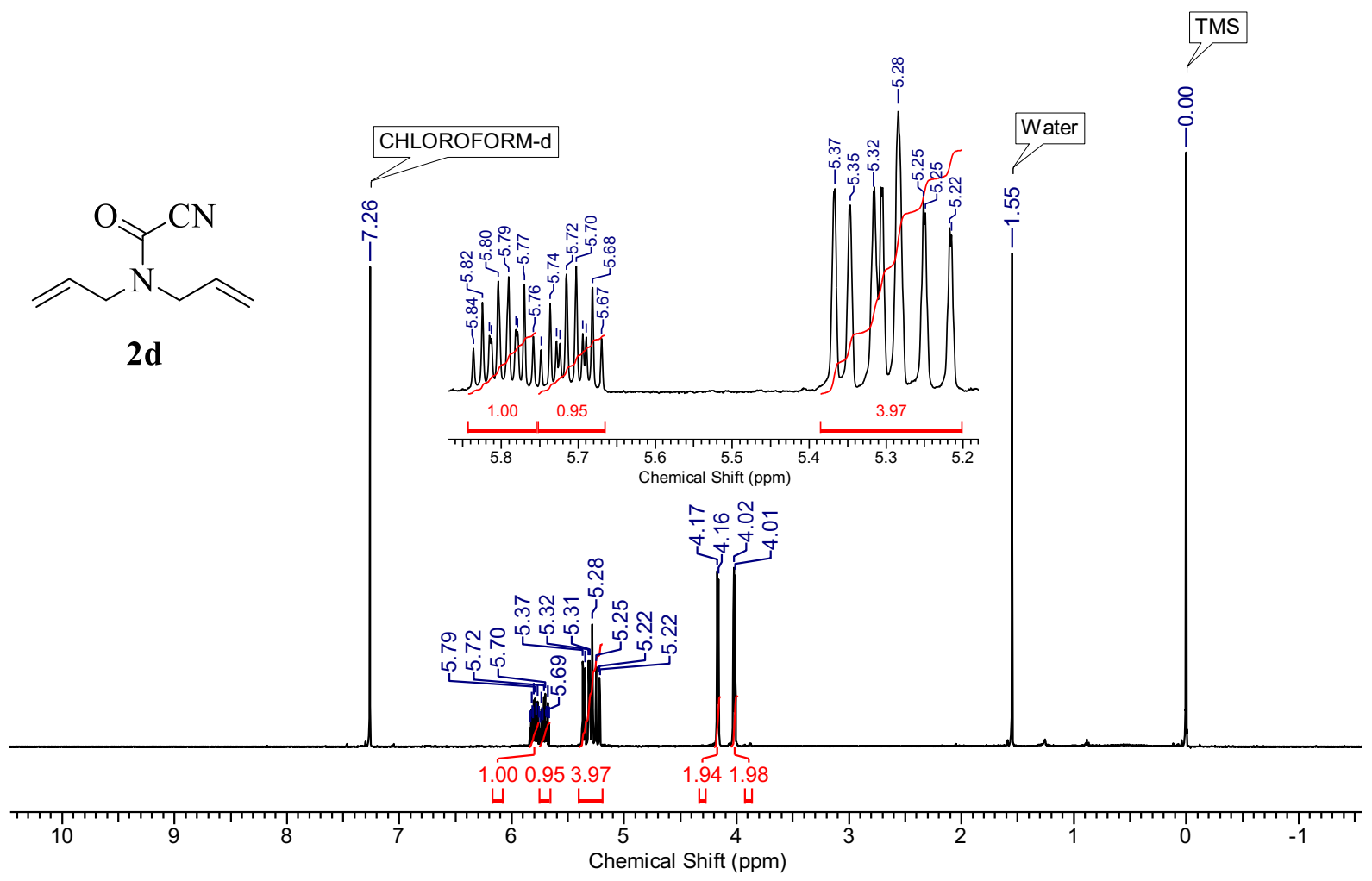

Figure S25. ${ }^{1} \mathrm{H}$ NMR spectrum of $\mathbf{2 d}$ in $\mathrm{CDCl}_{3}(500 \mathrm{MHz})$.

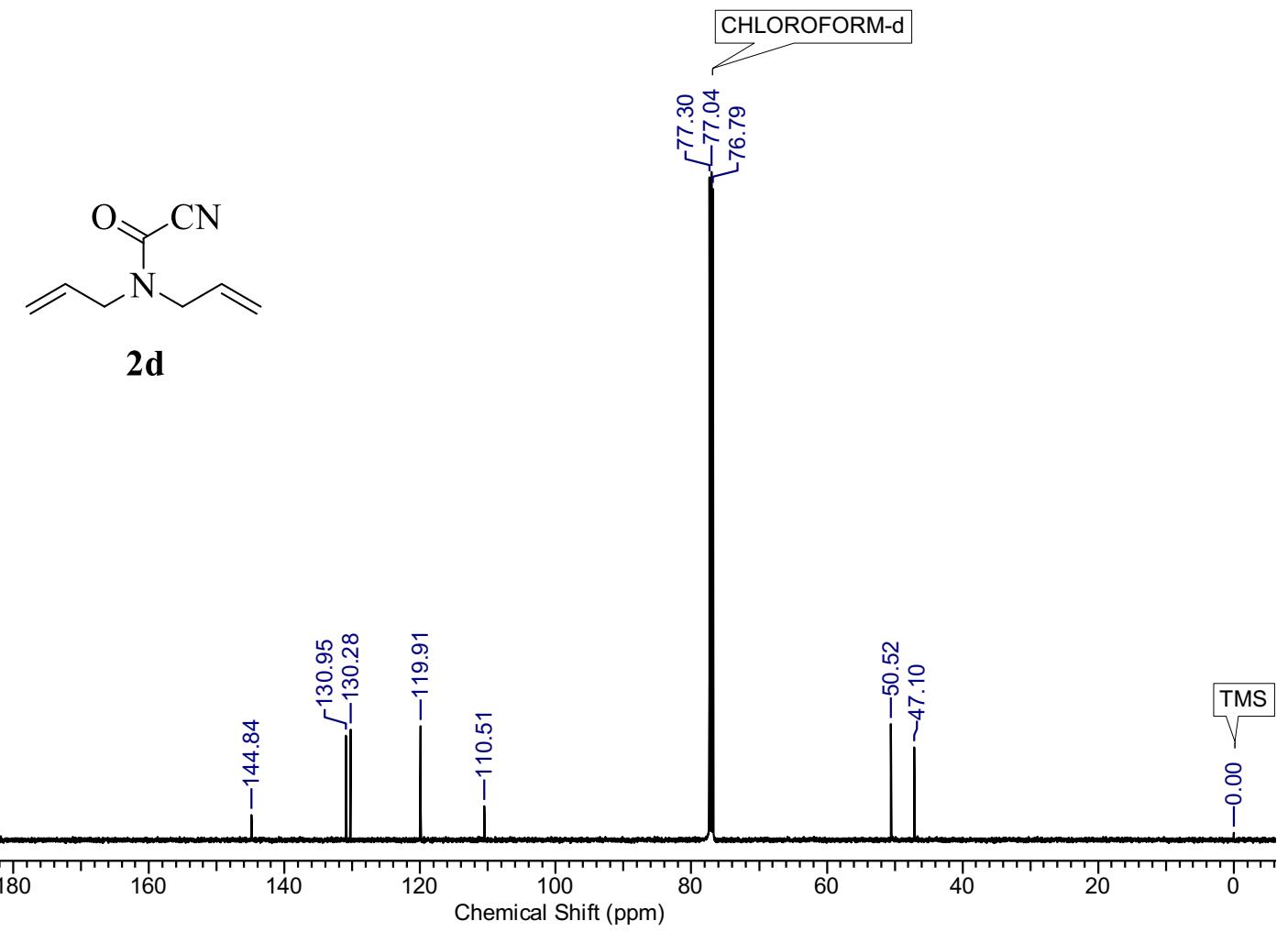

Figure S26. ${ }^{13} \mathrm{C}\left\{{ }^{1} \mathrm{H}\right\}$ NMR spectrum of $\mathbf{2 d}$ in $\mathrm{CDCl}_{3}(125 \mathrm{MHz})$. 


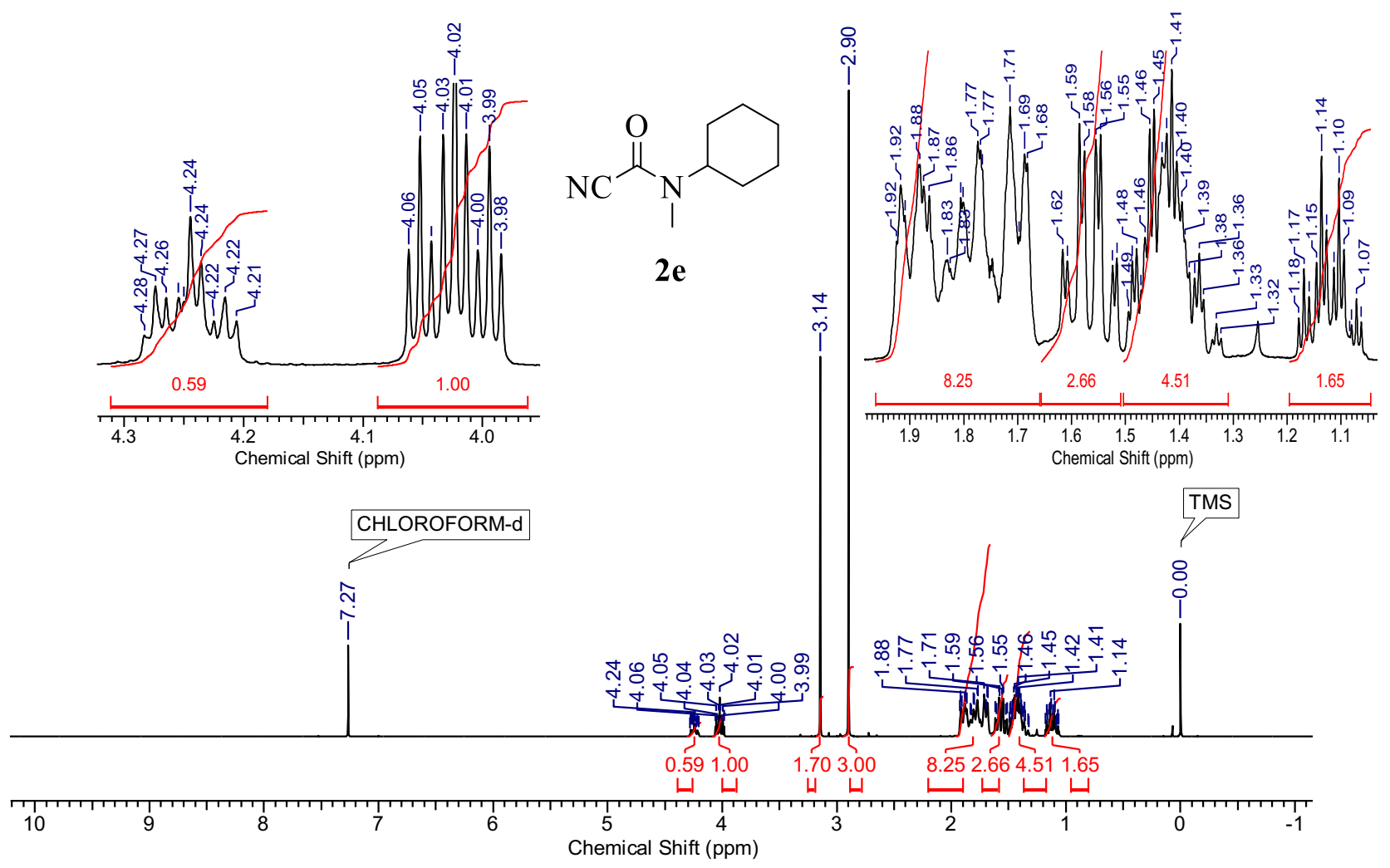

Figure S27. ${ }^{1} \mathrm{H}$ NMR spectrum of $2 \mathrm{e}$ in $\mathrm{CDCl}_{3}(400 \mathrm{MHz})$.

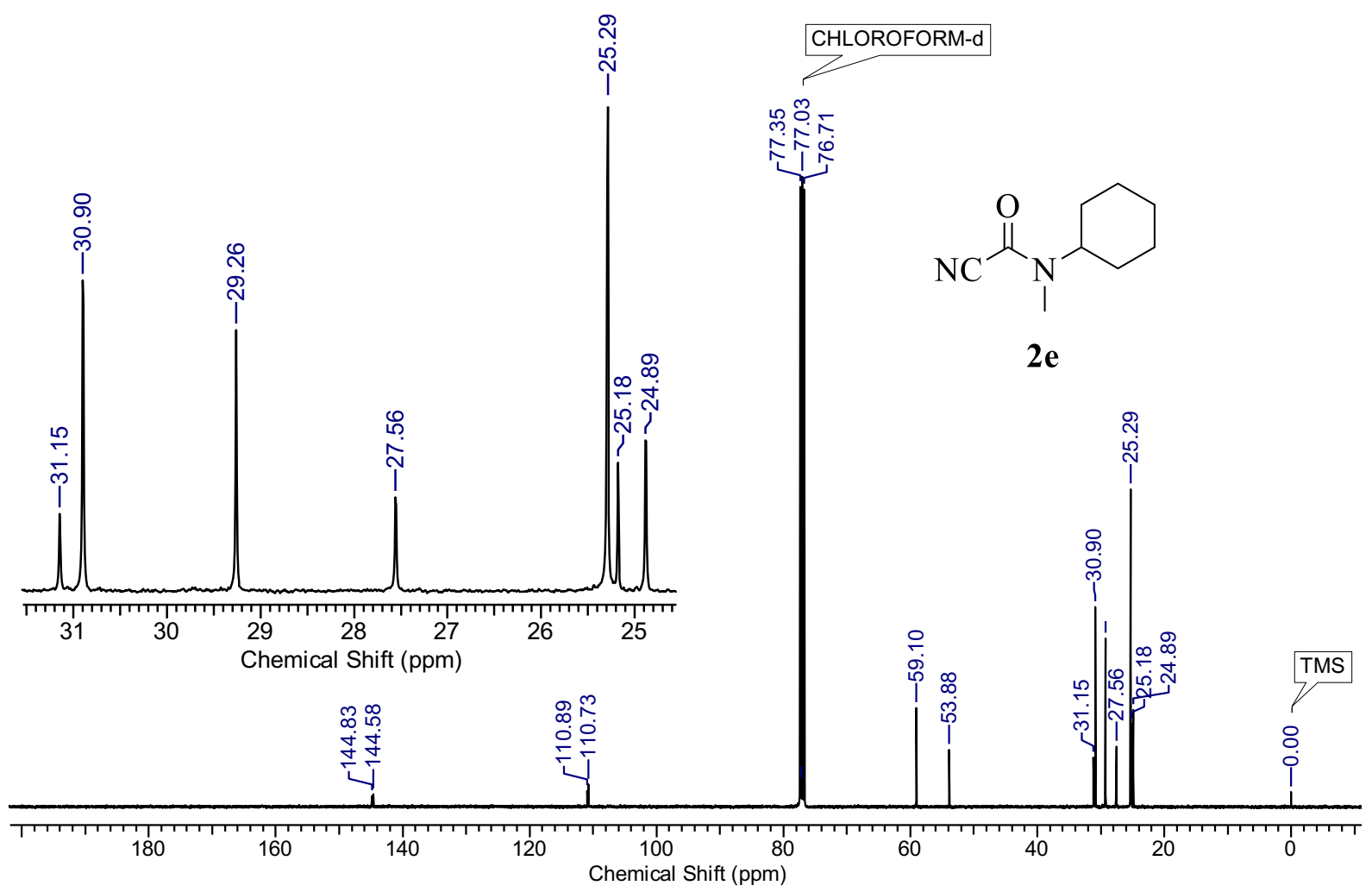

Figure S28. ${ }^{13} \mathrm{C}\left\{{ }^{1} \mathrm{H}\right\}$ NMR spectrum of $2 \mathrm{e}$ in $\mathrm{CDCl}_{3}(100 \mathrm{MHz})$. 


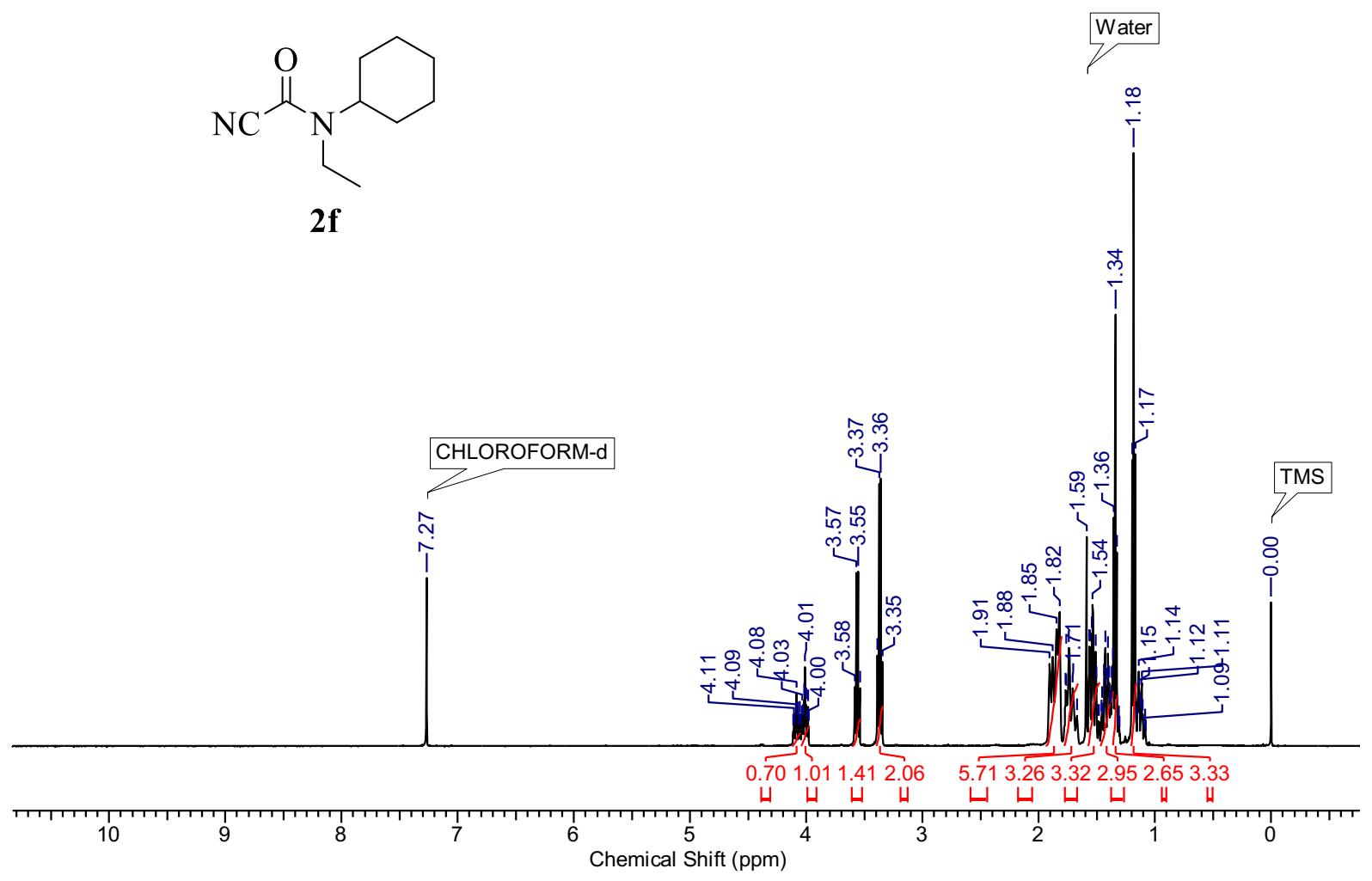

Figure S29. ${ }^{1} \mathrm{H}$ NMR spectrum of $2 \mathrm{f}$ in $\mathrm{CDCl}_{3}(500 \mathrm{MHz})$.

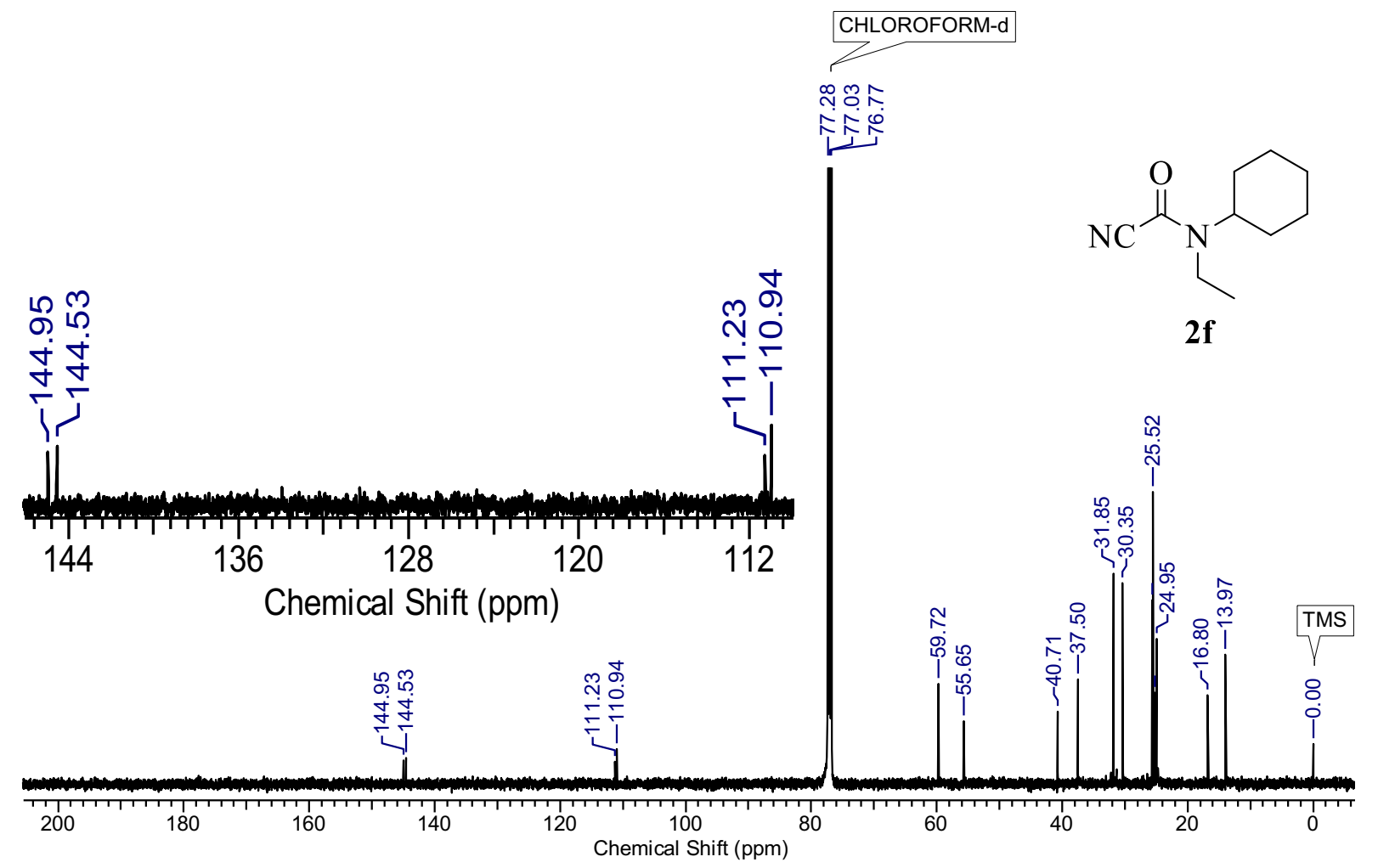

Figure S30. ${ }^{13} \mathrm{C}\left\{{ }^{1} \mathrm{H}\right\}$ NMR spectrum of $2 \mathbf{f}$ in $\mathrm{CDCl}_{3}(125 \mathrm{MHz})$. 


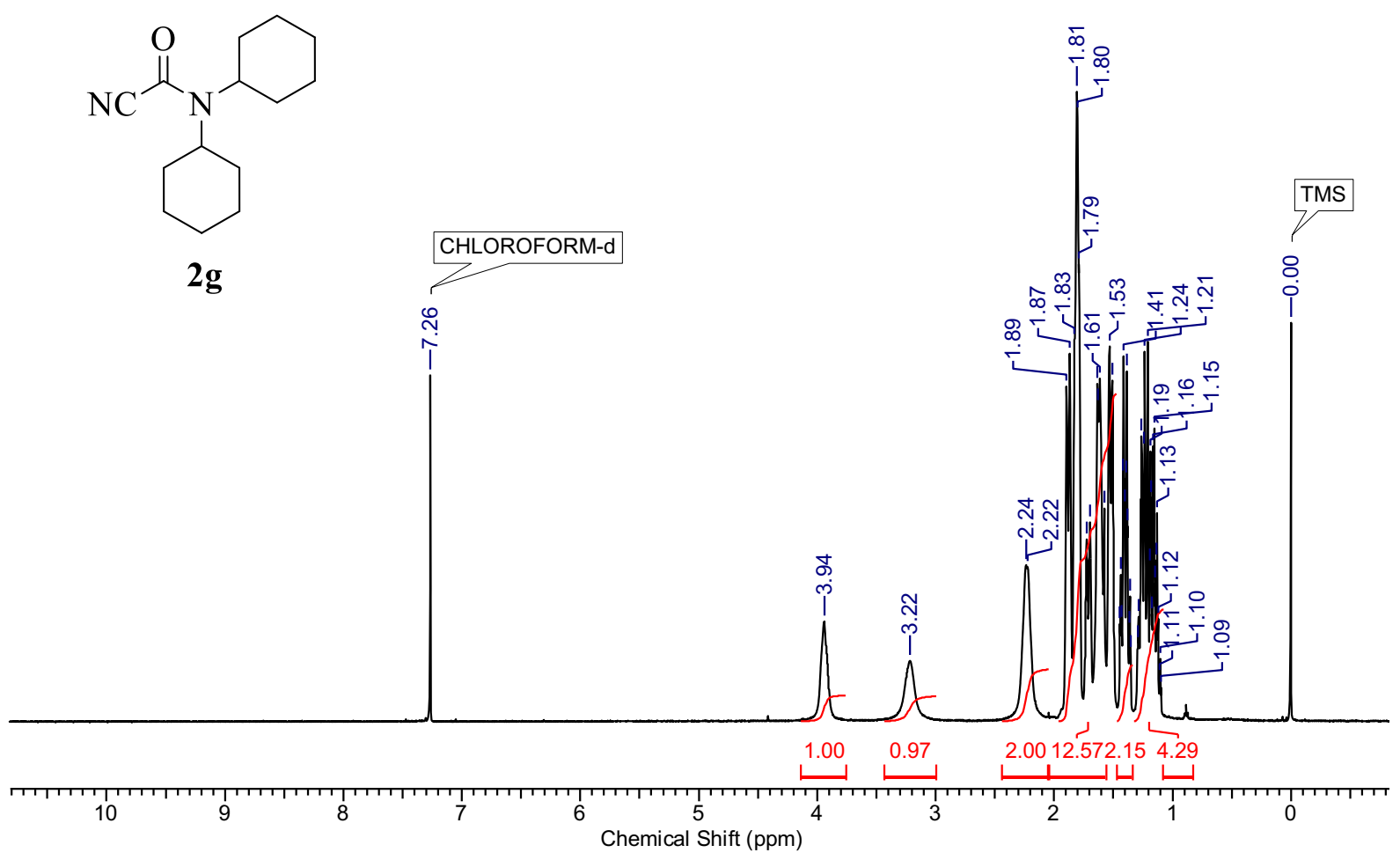

Figure S31. ${ }^{1} \mathrm{H}$ NMR spectrum of $\mathbf{2 g}$ in $\mathrm{CDCl}_{3}(500 \mathrm{MHz})$.

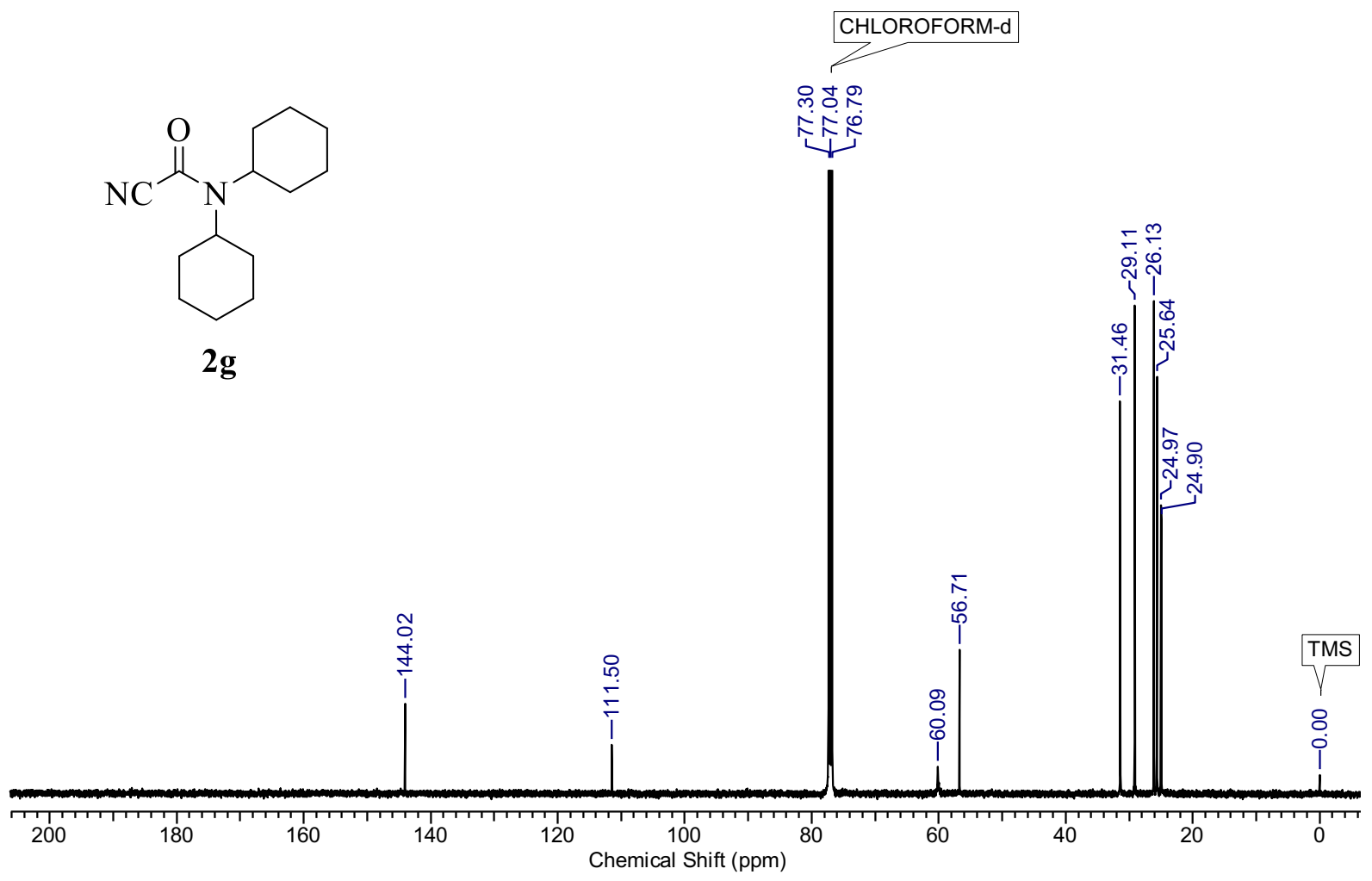

Figure S32. ${ }^{13} \mathrm{C}\left\{{ }^{1} \mathrm{H}\right\}$ NMR spectrum of $\mathbf{2} \mathbf{g}$ in $\mathrm{CDCl}_{3}(125 \mathrm{MHz})$. 


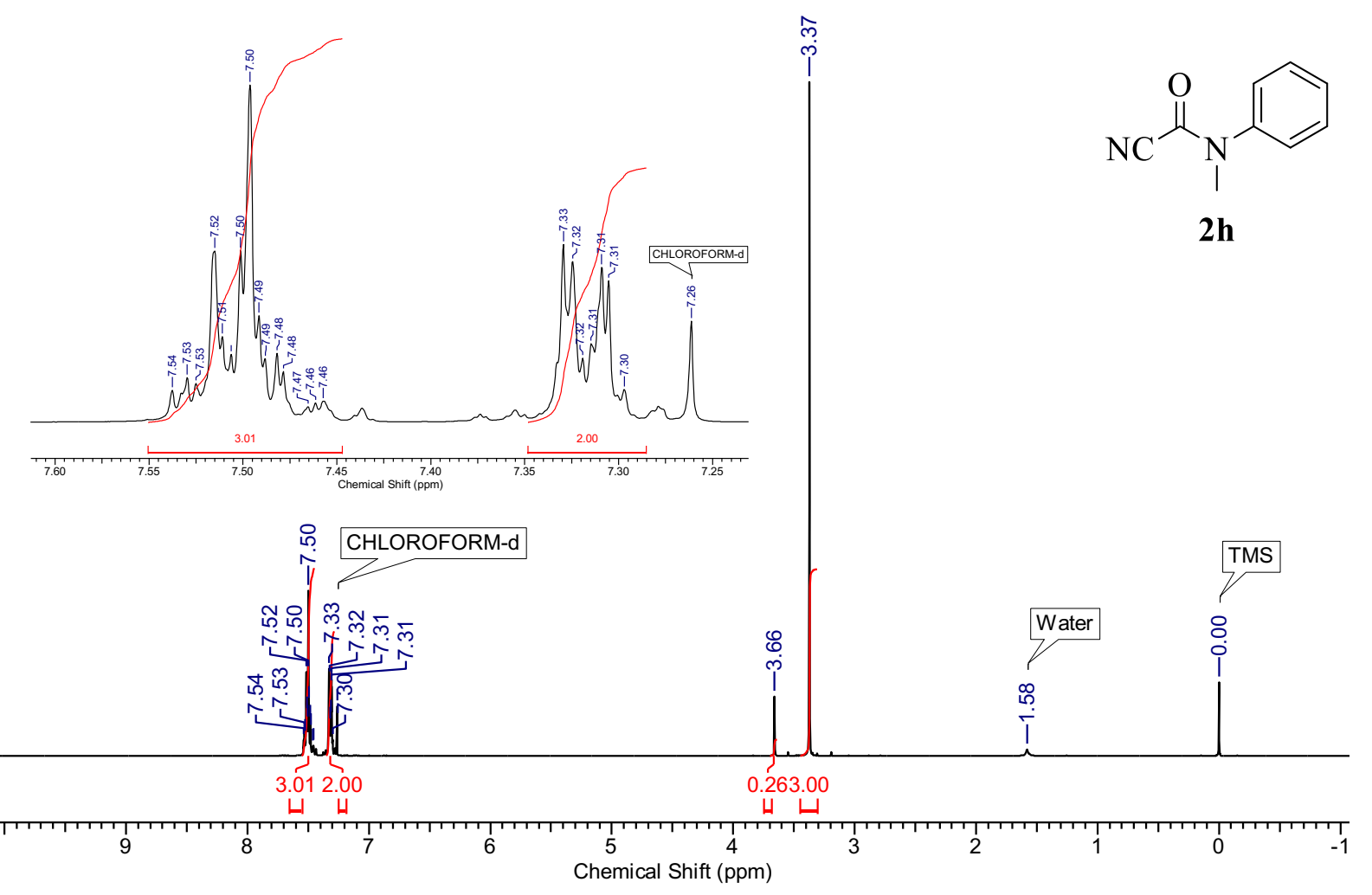

Figure S33. ${ }^{1} \mathrm{H}$ NMR spectrum of $\mathbf{2} \mathbf{h}$ in $\mathrm{CDCl}_{3}(400 \mathrm{MHz})$.

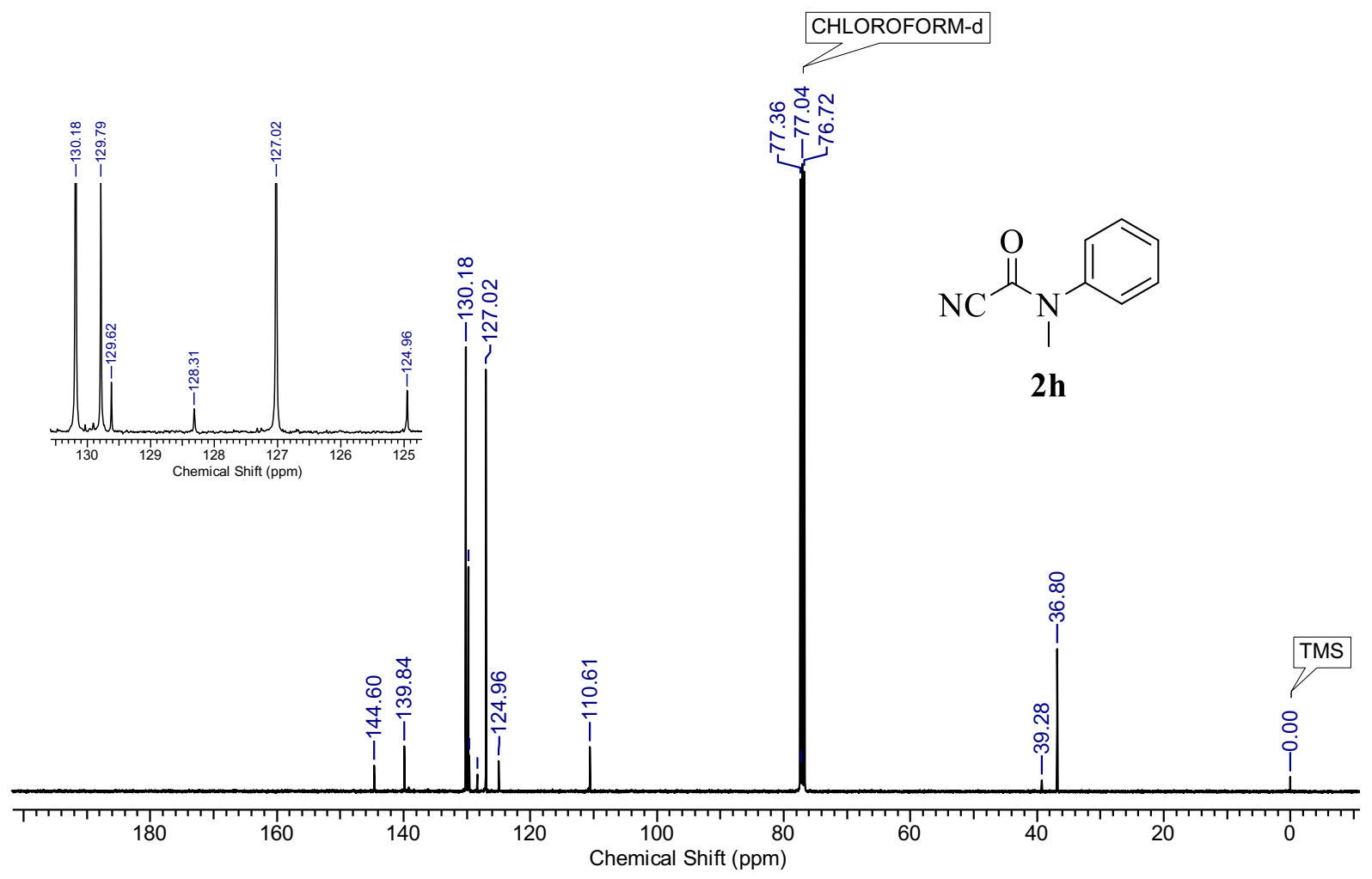

Figure S34. ${ }^{13} \mathrm{C}\left\{{ }^{1} \mathrm{H}\right\}$ NMR spectrum of $\mathbf{2} \mathbf{h}$ in $\mathrm{CDCl}_{3}(100 \mathrm{MHz})$. 


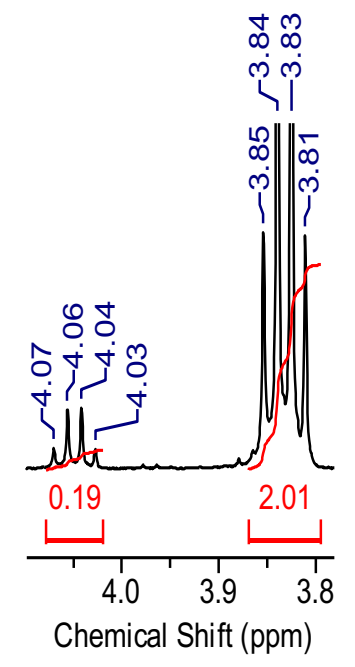<smiles>CCN(C([N])=O)c1ccccc1</smiles>

$2 \mathbf{i}$
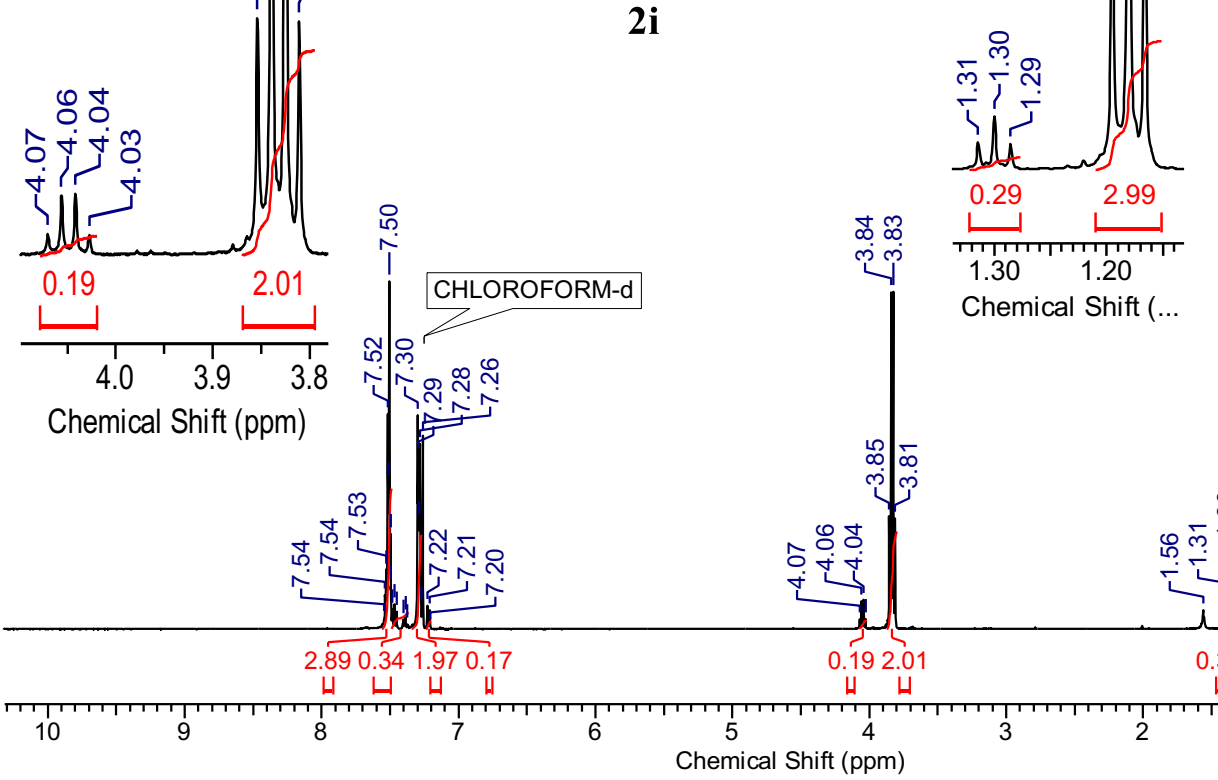

Figure S35. ${ }^{1} \mathrm{H}$ NMR spectrum of $2 \mathbf{i}$ in $\mathrm{CDCl}_{3}(500 \mathrm{MHz})$.

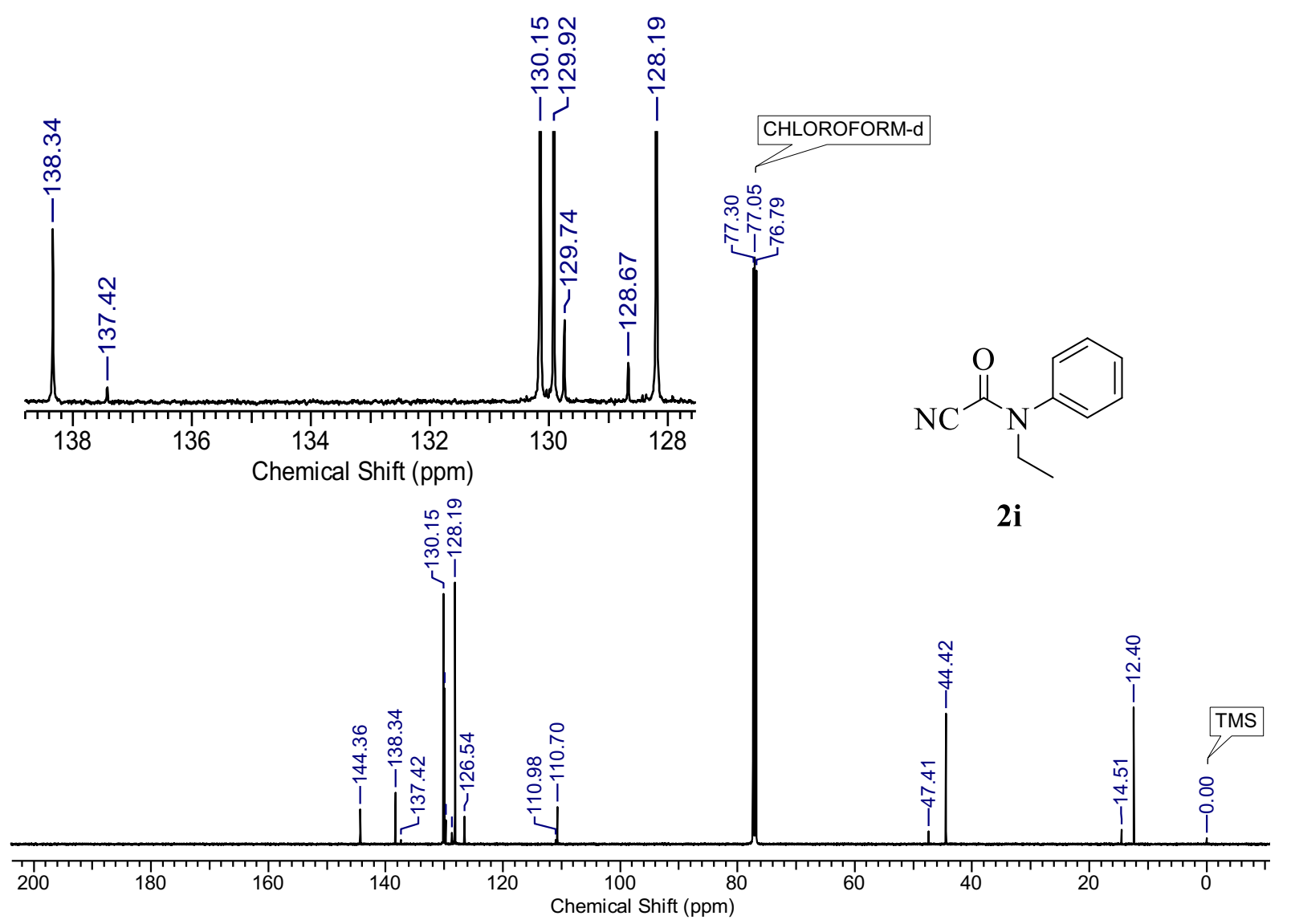

Figure S36. ${ }^{13} \mathrm{C}\left\{{ }^{1} \mathrm{H}\right\}$ NMR spectrum of $\mathbf{2} \mathbf{i}$ in $\mathrm{CDCl}_{3}(125 \mathrm{MHz})$. 


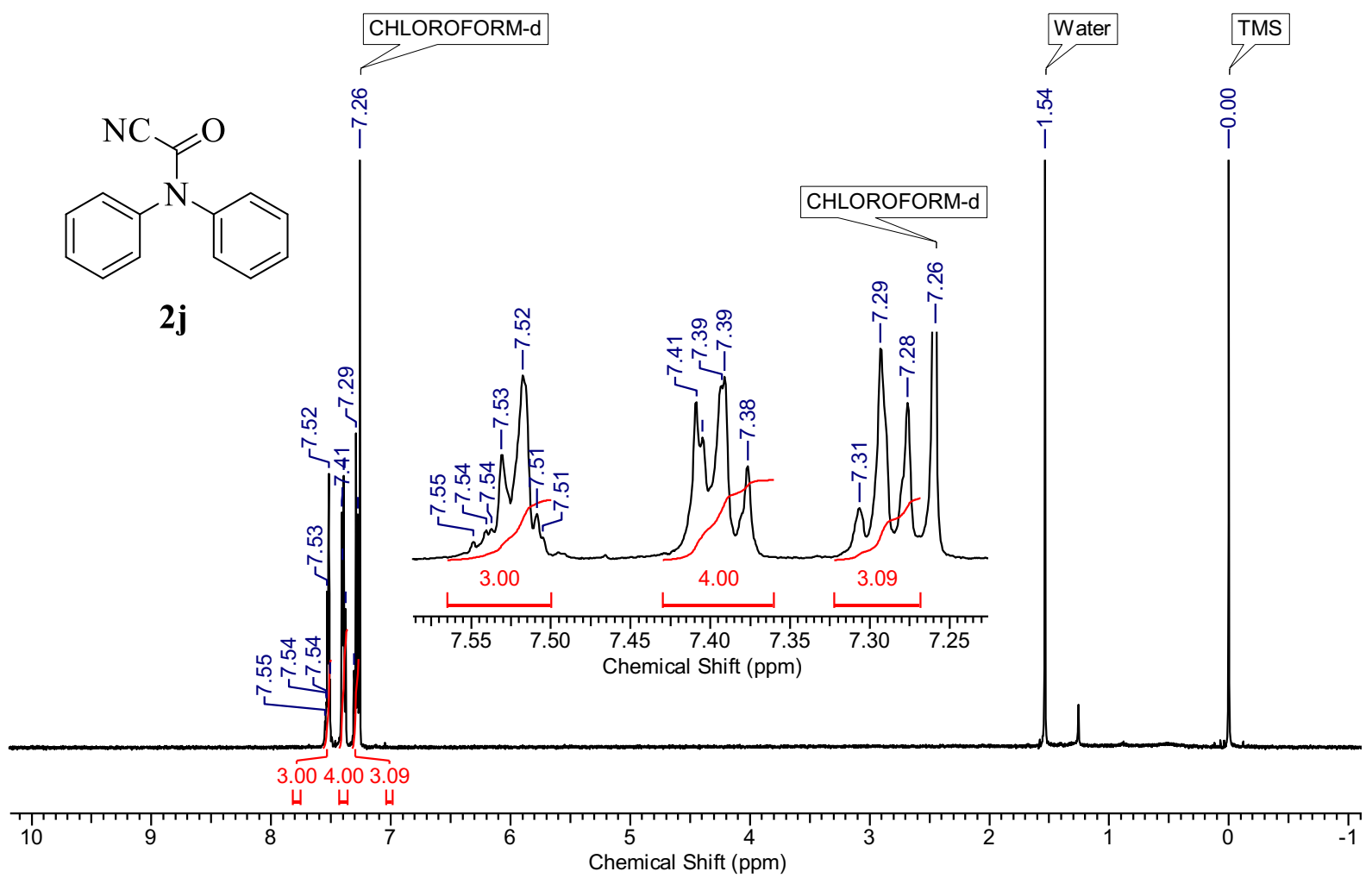

Figure S37. ${ }^{1} \mathrm{H}$ NMR spectrum of $2 \mathbf{j}$ in $\mathrm{CDCl}_{3}(500 \mathrm{MHz})$.

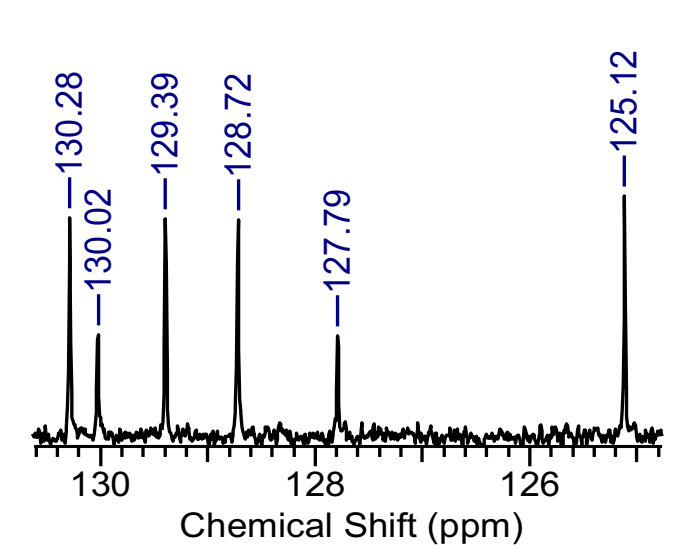

CHLOROFORM-d

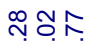

年尔<smiles>N#CC(=O)N(c1ccccc1)c1ccccc1</smiles>

$2 \mathbf{j}$

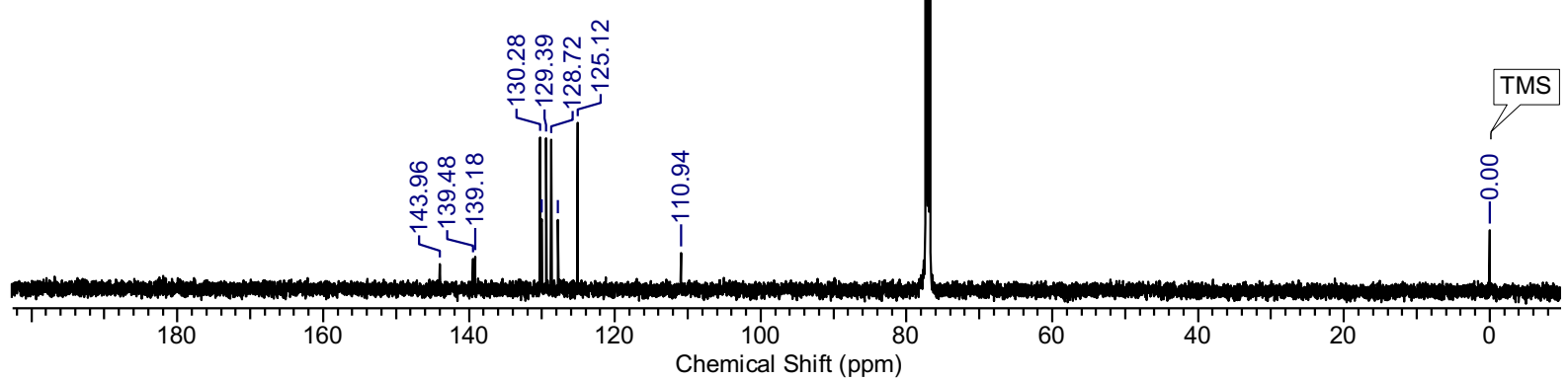

Figure S38. ${ }^{13} \mathrm{C}\left\{{ }^{1} \mathrm{H}\right\}$ NMR spectrum of $2 \mathrm{j}$ in $\mathrm{CDCl}_{3}(125 \mathrm{MHz})$. 


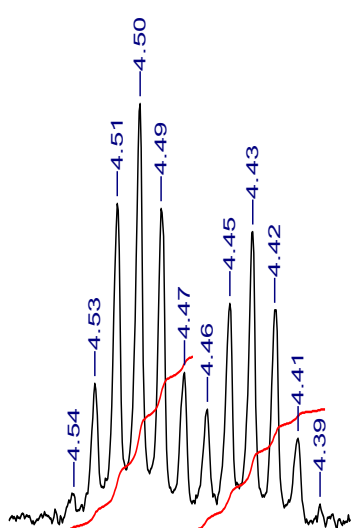

$1.00 \quad 0.70$

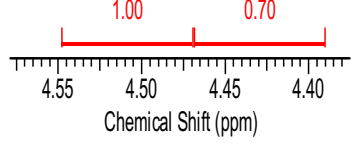<smiles>CCN(C(C)=O)C(C)C</smiles>

$2 \mathbf{k}$

\section{CHLOROFORM-d}

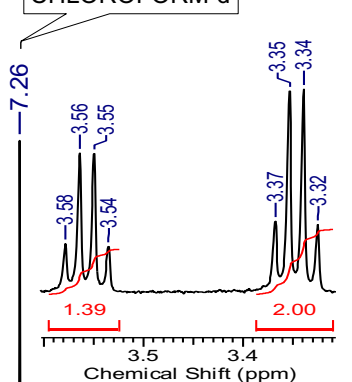

Chemical Shift (ppm)

$$
\text { ก เก }
$$
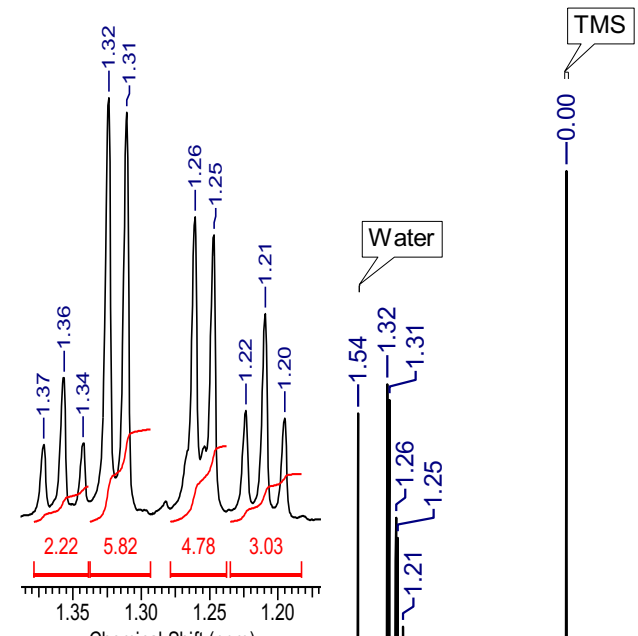

Chemical Shift (ppm)

ले से

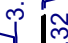

ĩ

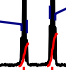

392.00

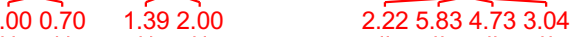

10

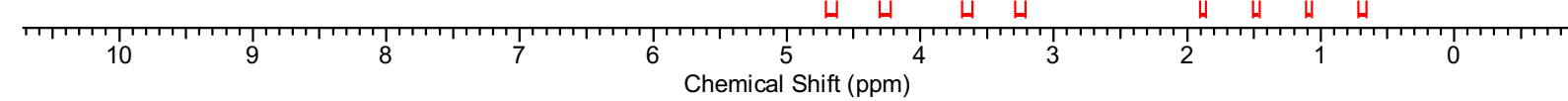

Figure S39. ${ }^{1} \mathrm{H}$ NMR spectrum of $\mathbf{2} \mathbf{k}$ in $\mathrm{CDCl}_{3}(500 \mathrm{MHz})$.

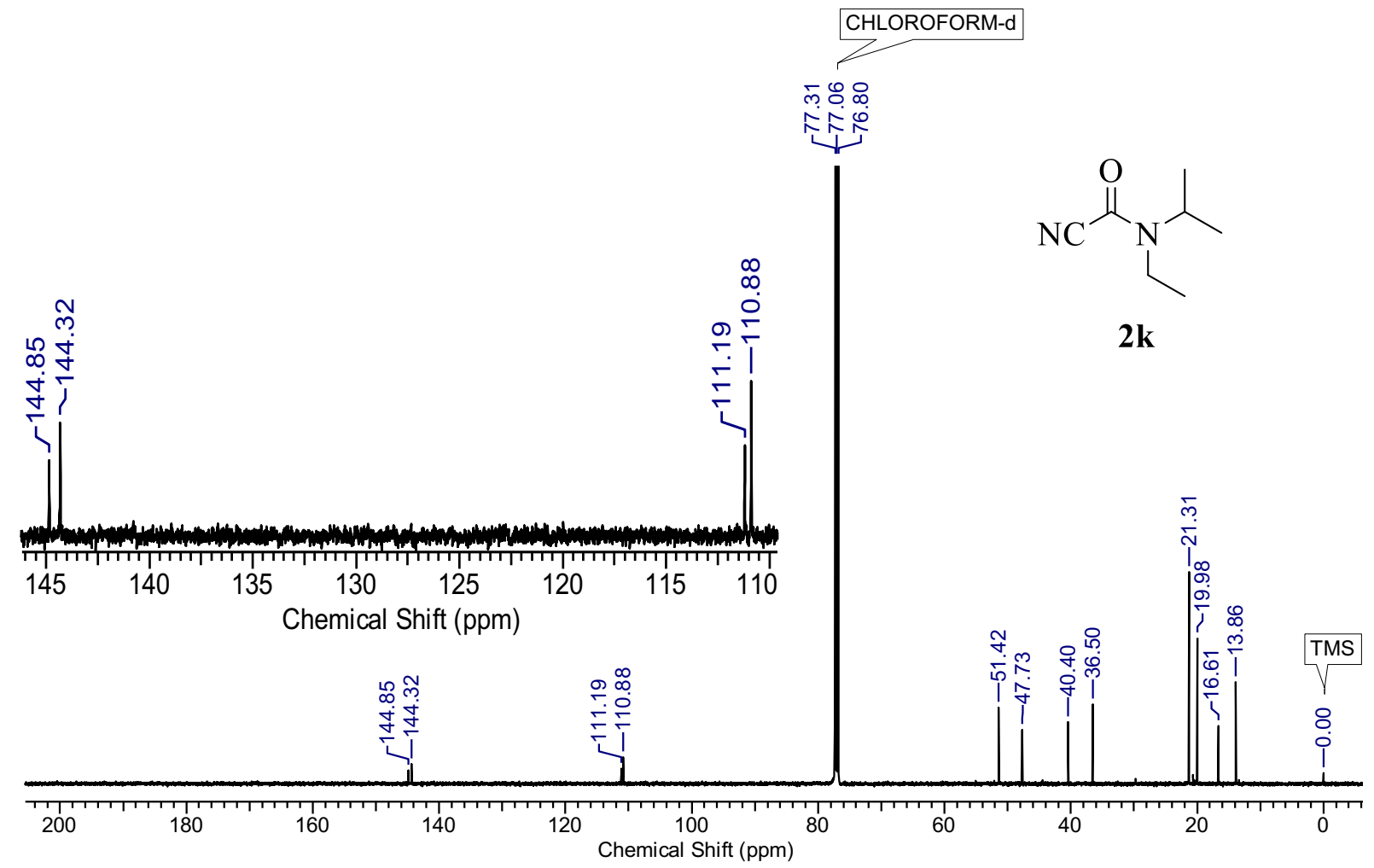

Figure S40. ${ }^{13} \mathrm{C}\left\{{ }^{1} \mathrm{H}\right\}$ NMR spectrum of $\mathbf{2} \mathbf{k}$ in $\mathrm{CDCl}_{3}(125 \mathrm{MHz})$. 

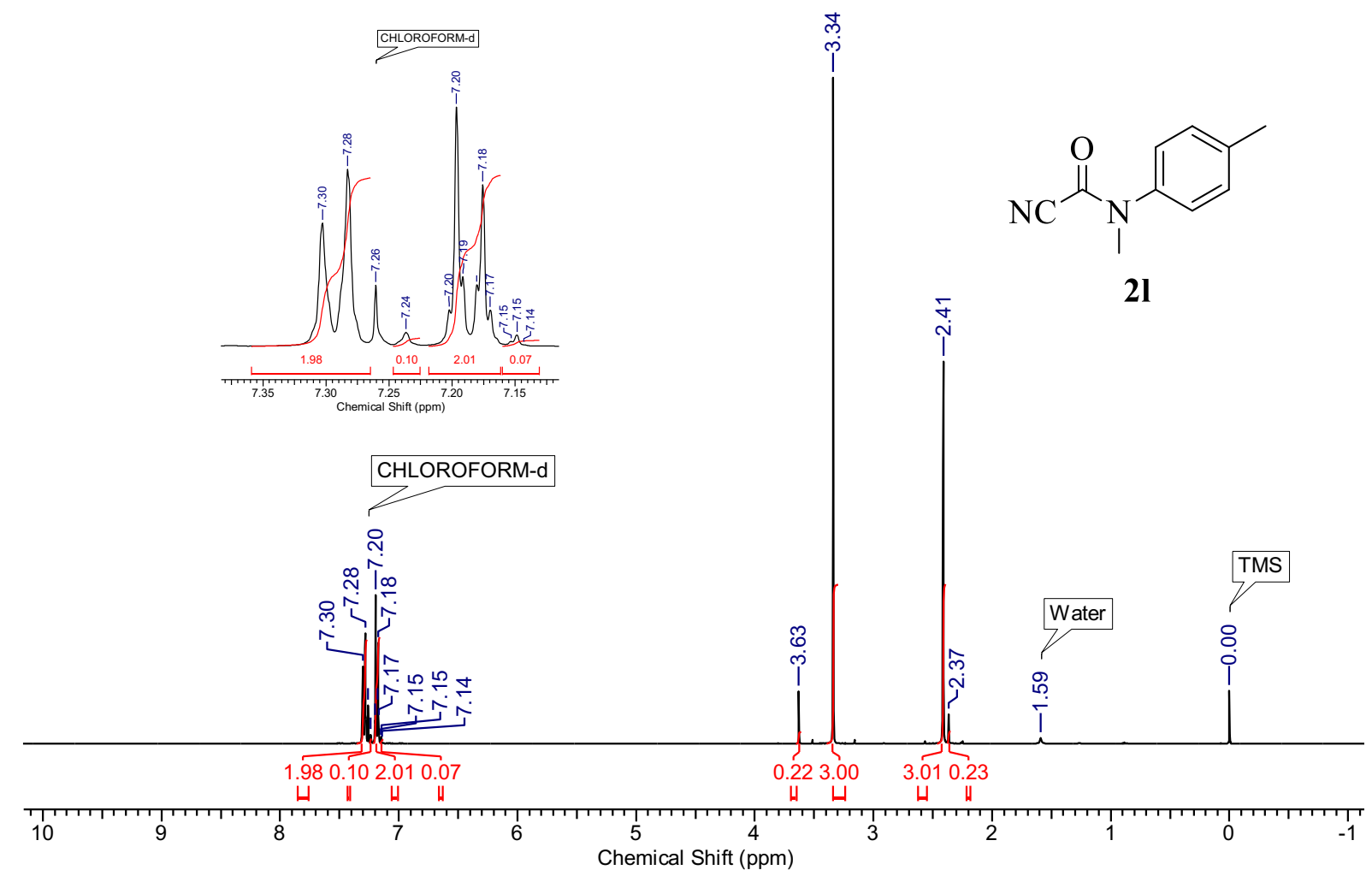

Figure S41. ${ }^{1} \mathrm{H}$ NMR spectrum of $2 \mathrm{I}$ in $\mathrm{CDCl}_{3}(400 \mathrm{MHz})$.

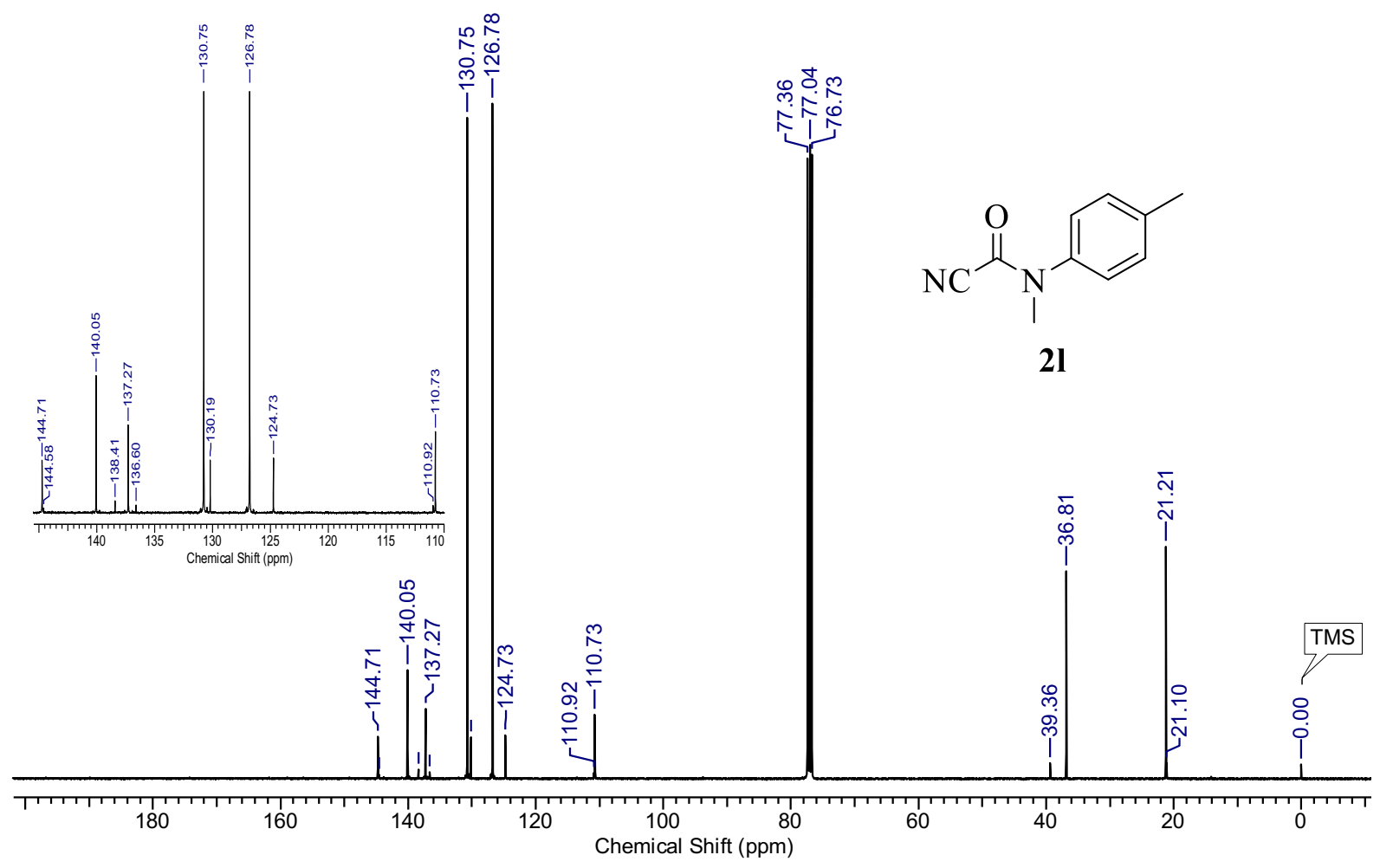

Figure S42. ${ }^{13} \mathrm{C}\left\{{ }^{1} \mathrm{H}\right\}$ NMR spectrum of $2 \mathrm{I}$ in $\mathrm{CDCl}_{3}(100 \mathrm{MHz})$. 


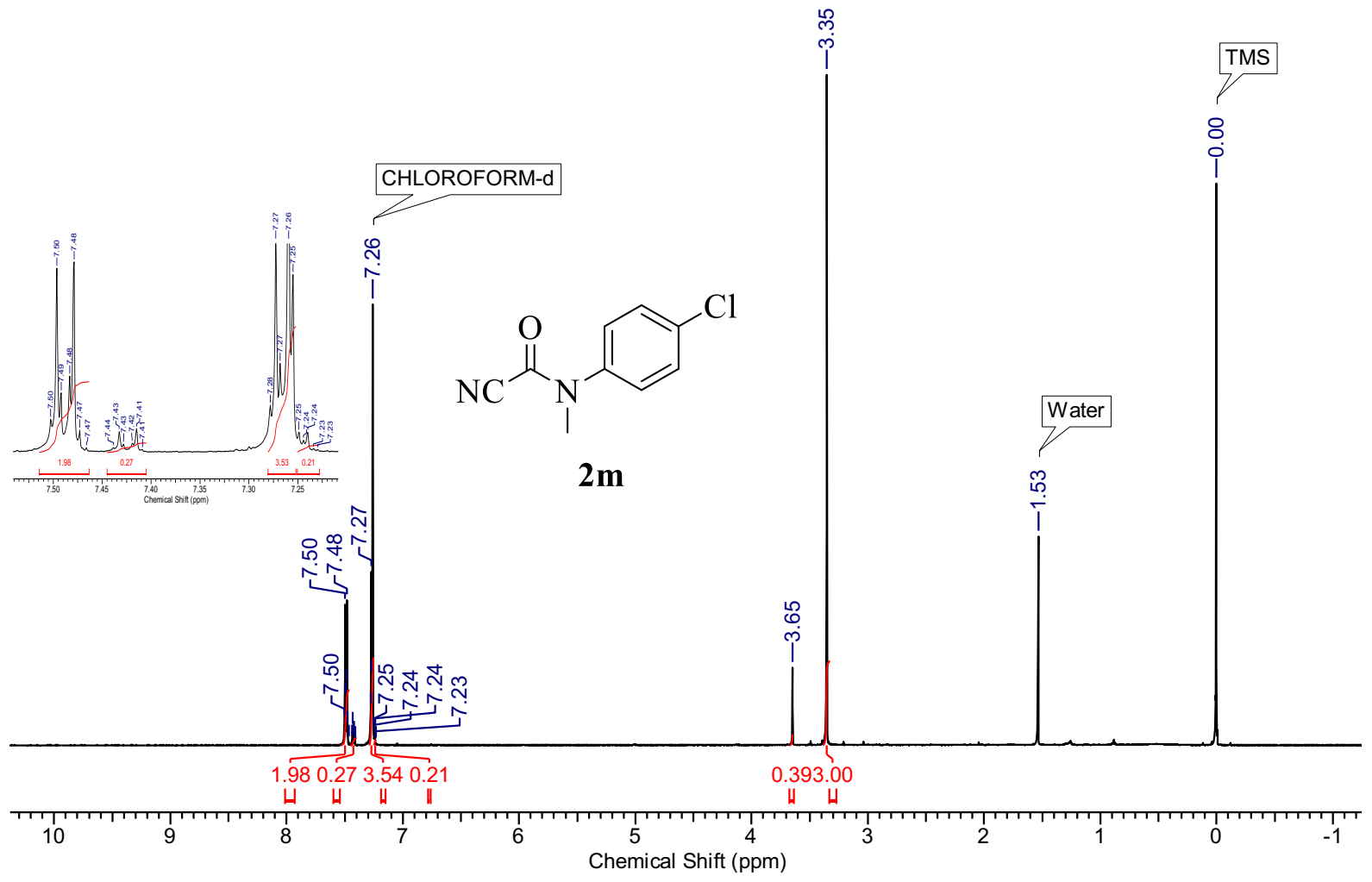

Figure S43. ${ }^{1} \mathrm{H}$ NMR spectrum of $2 \mathrm{~m}$ in $\mathrm{CDCl}_{3}(500 \mathrm{MHz})$.

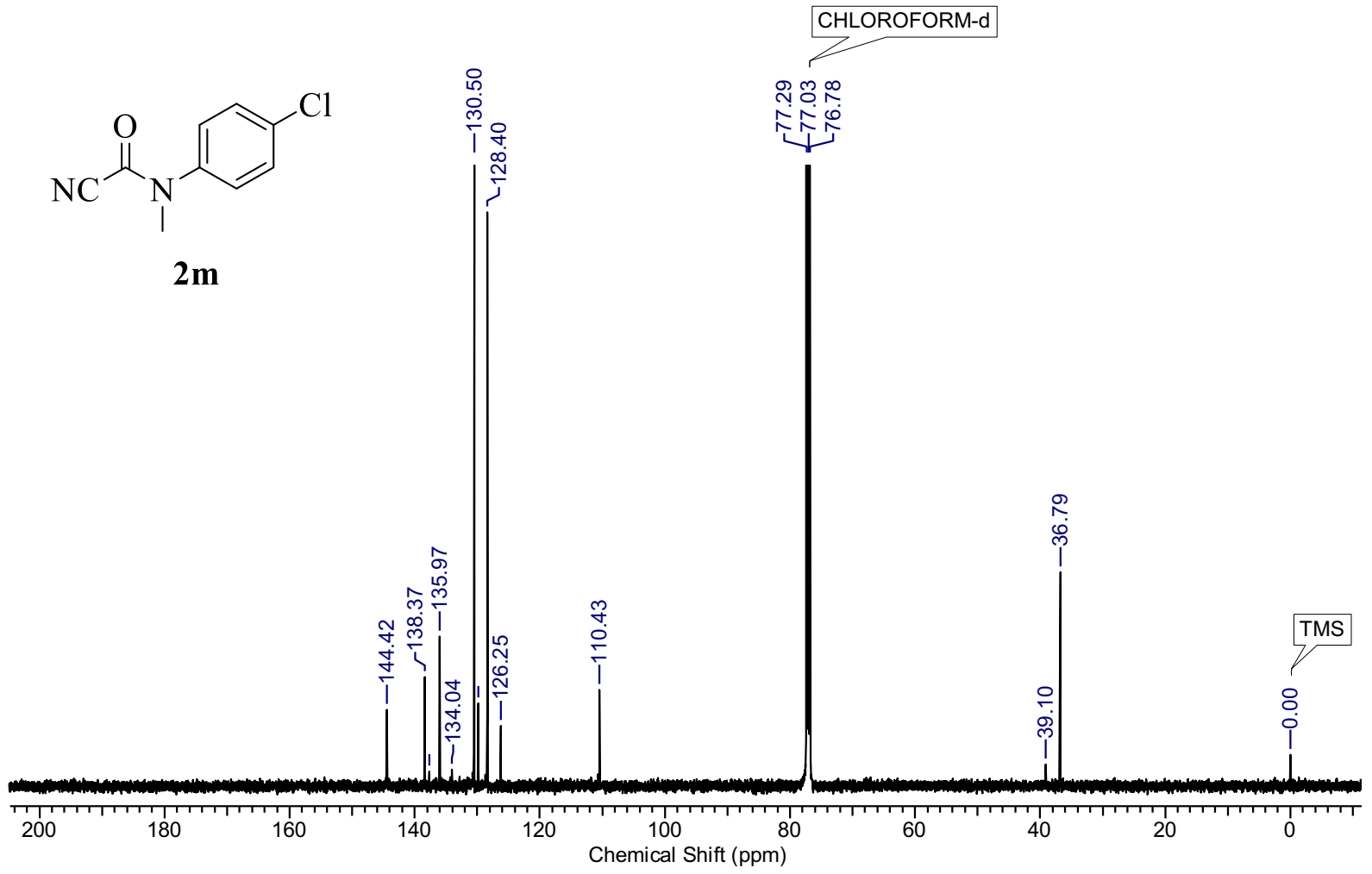

Figure S44. ${ }^{13} \mathrm{C}\left\{{ }^{1} \mathrm{H}\right\}$ NMR spectrum of $2 \mathrm{~m}$ in $\mathrm{CDCl}_{3}(125 \mathrm{MHz})$. 


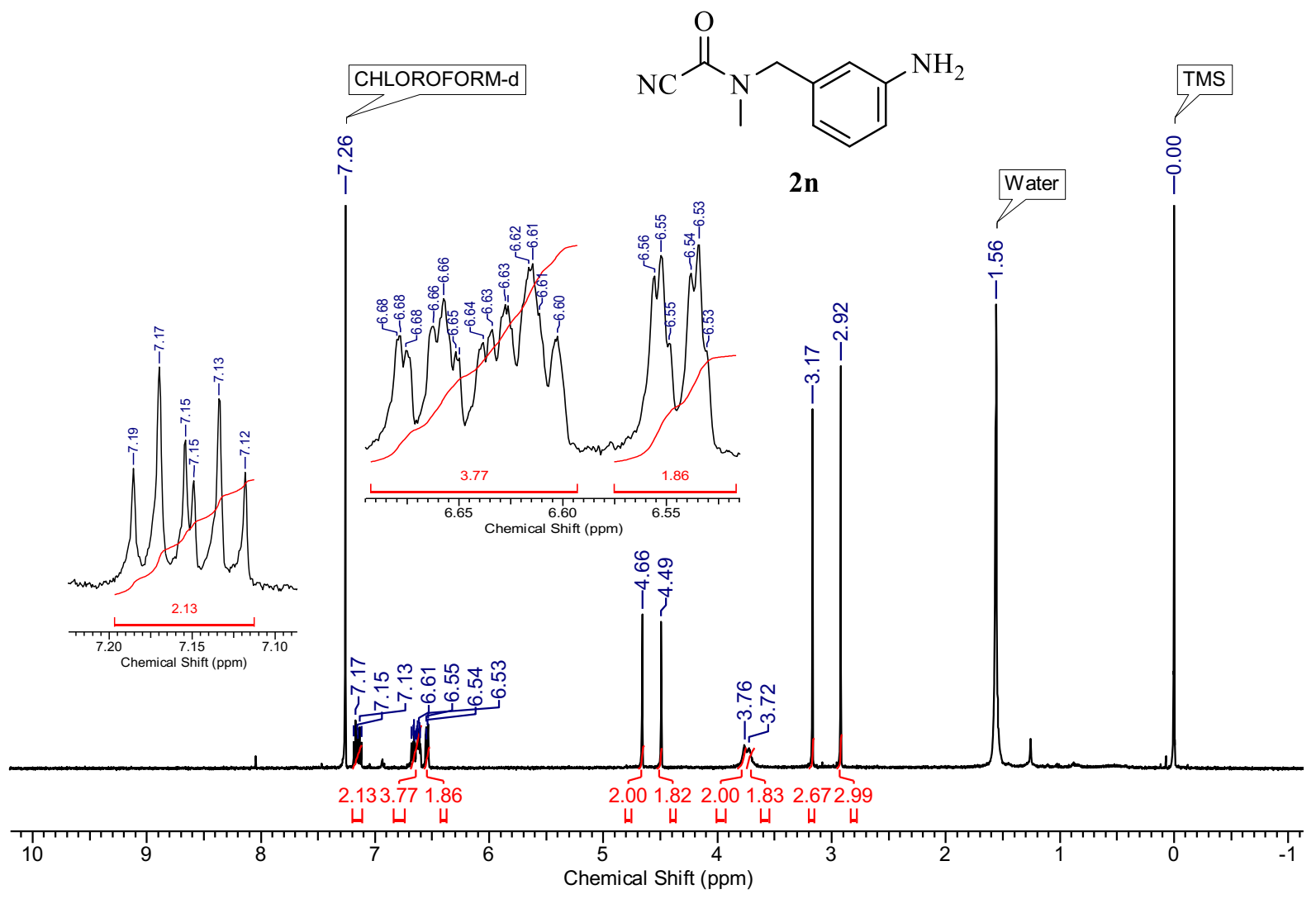

Figure S45. ${ }^{1} \mathrm{H}$ NMR spectrum of $\mathbf{2 n}$ in $\mathrm{CDCl}_{3}(500 \mathrm{MHz})$.

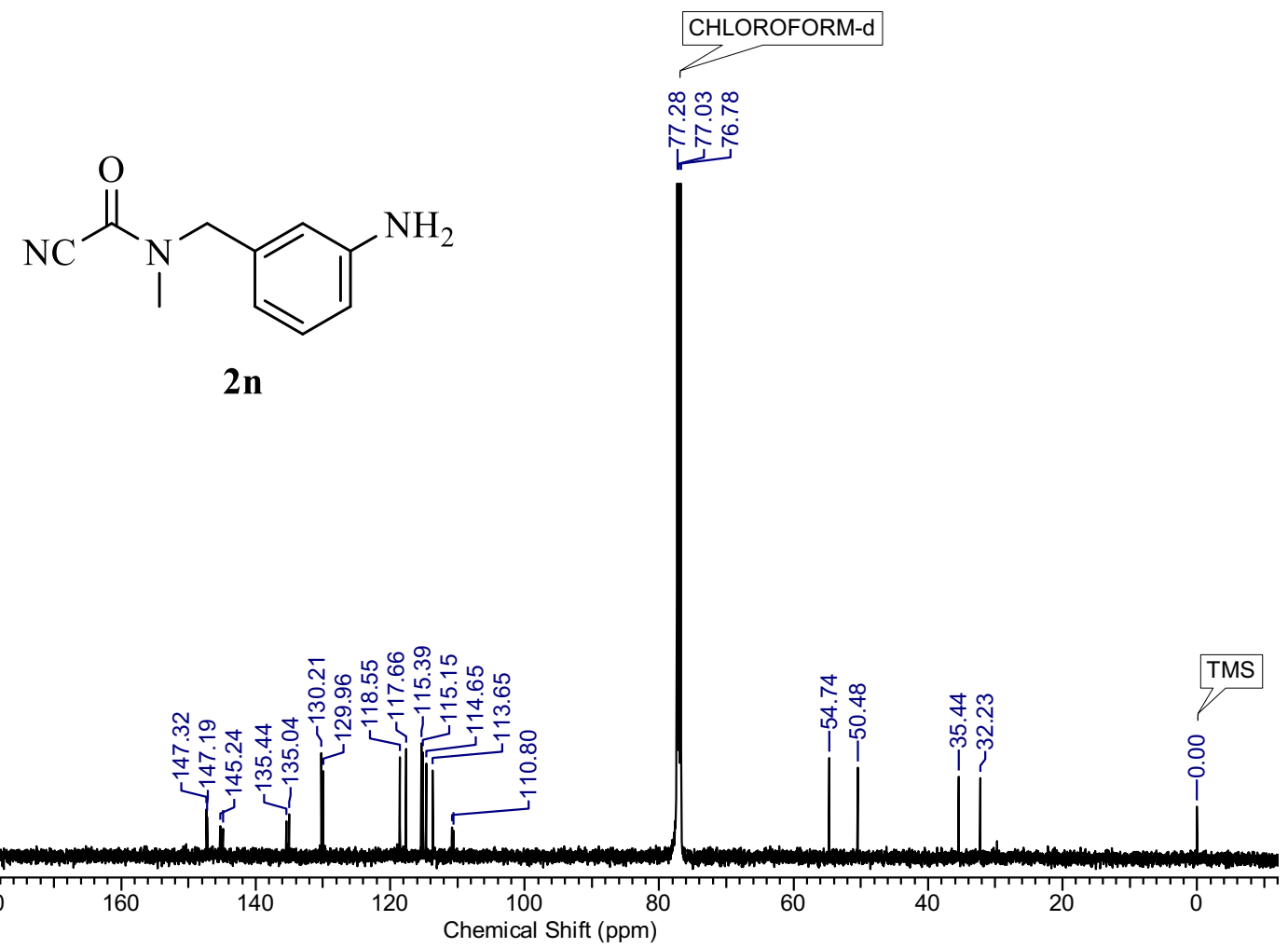

Figure S46. ${ }^{13} \mathrm{C}\left\{{ }^{1} \mathrm{H}\right\}$ NMR spectrum of $\mathbf{2 n}$ in $\mathrm{CDCl}_{3}(125 \mathrm{MHz})$. 


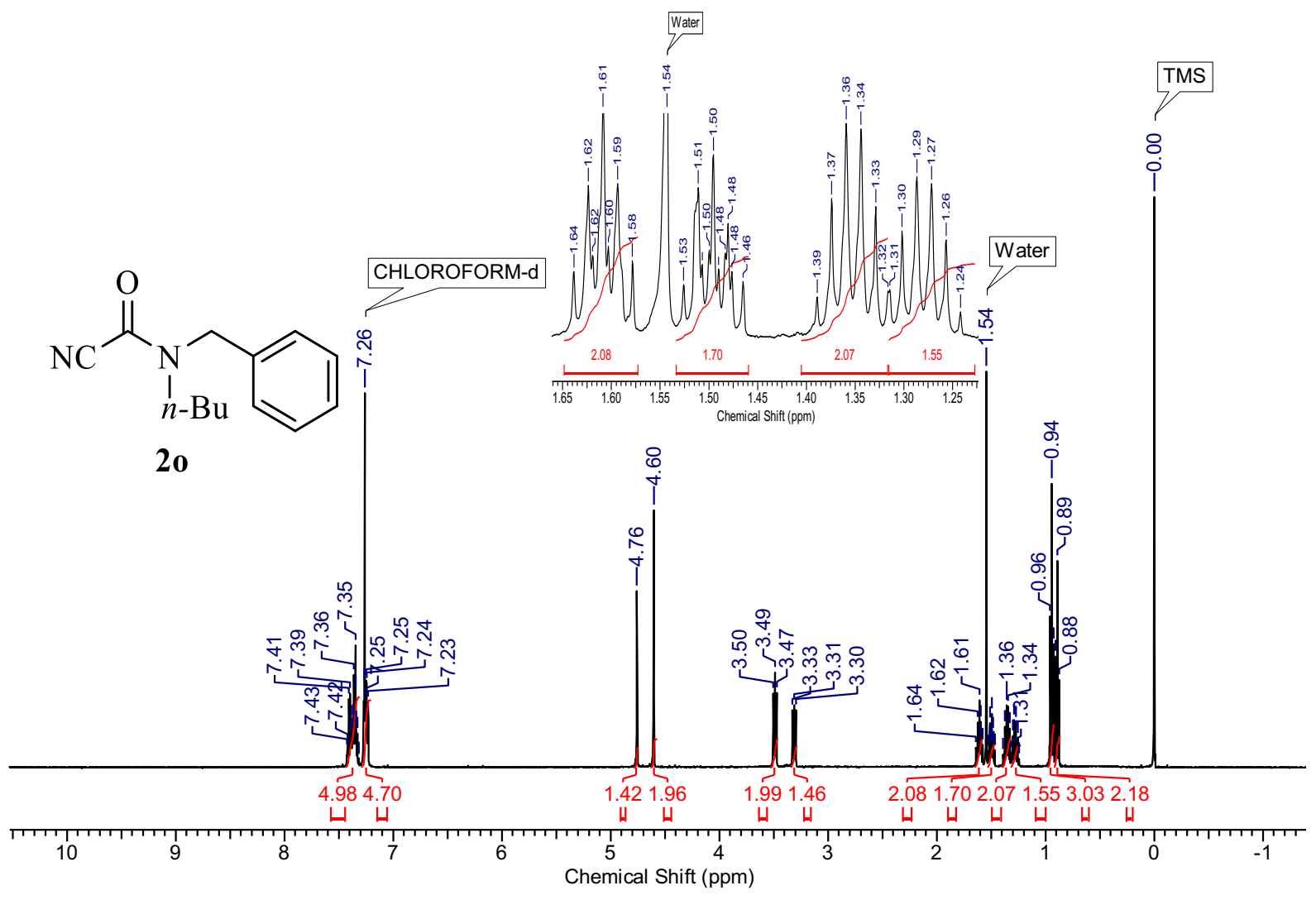

Figure S47. ${ }^{1} \mathrm{H}$ NMR spectrum of 20 in $\mathrm{CDCl}_{3}(500 \mathrm{MHz})$.

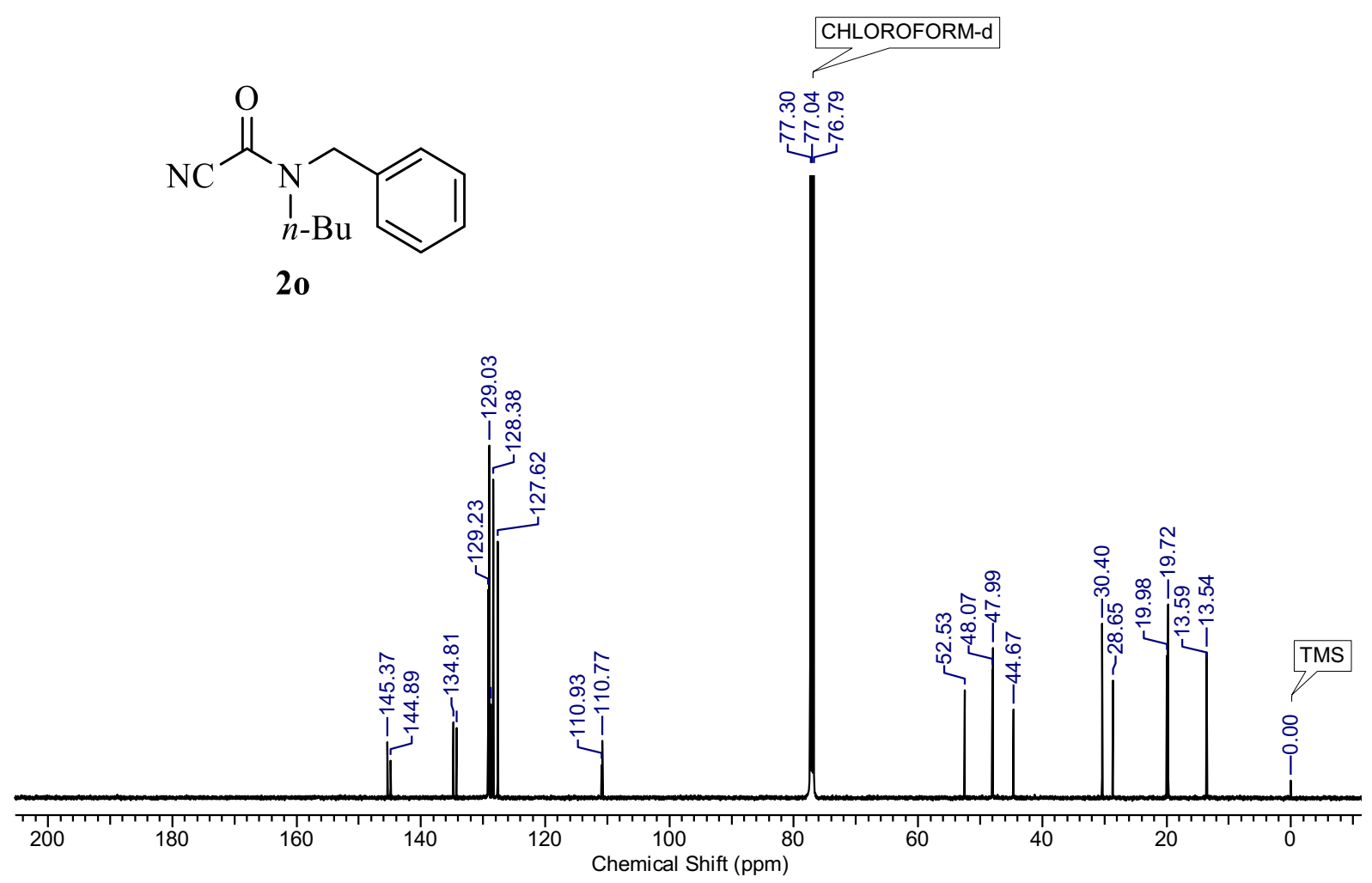

Figure S48. ${ }^{13} \mathrm{C}\left\{{ }^{1} \mathrm{H}\right\}$ NMR spectrum of 20 in $\mathrm{CDCl}_{3}(125 \mathrm{MHz})$. 


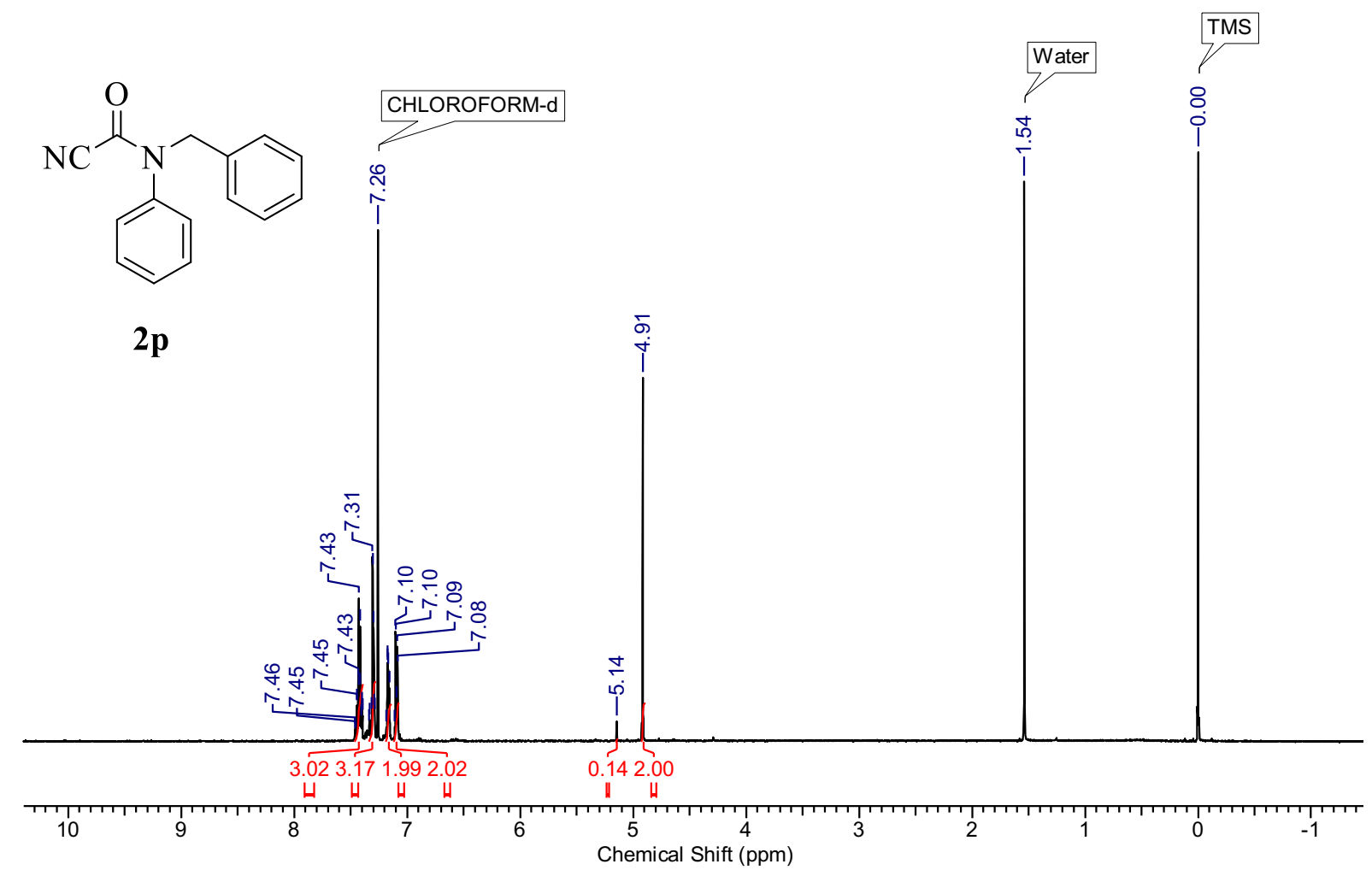

Figure S49. ${ }^{1} \mathrm{H}$ NMR spectrum of $2 p$ in $\mathrm{CDCl}_{3}(500 \mathrm{MHz})$.

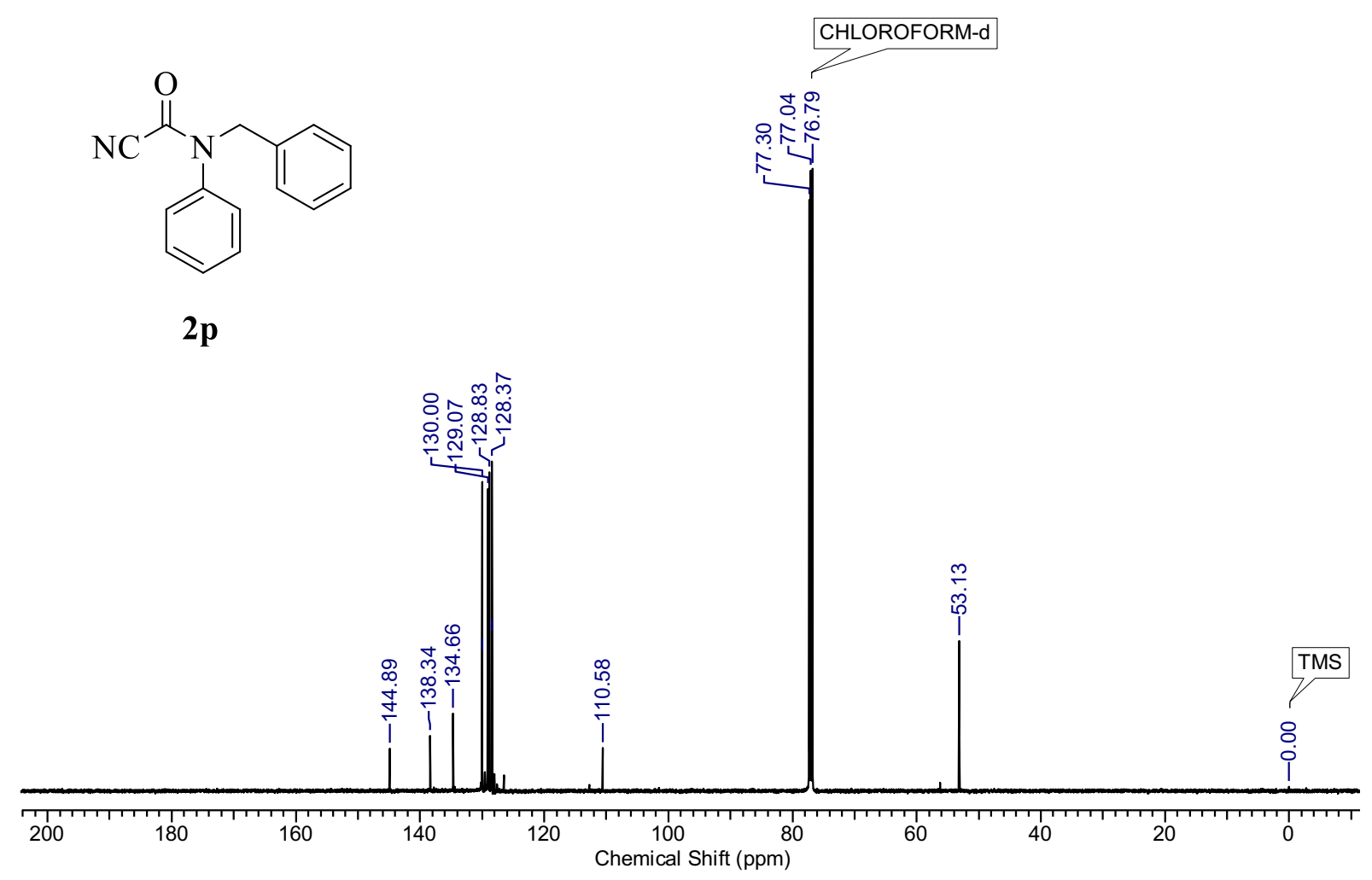

Figure S50. ${ }^{13} \mathrm{C}\left\{{ }^{1} \mathrm{H}\right\}$ NMR spectrum of $\mathbf{2 p}$ in $\mathrm{CDCl}_{3}(125 \mathrm{MHz})$. 


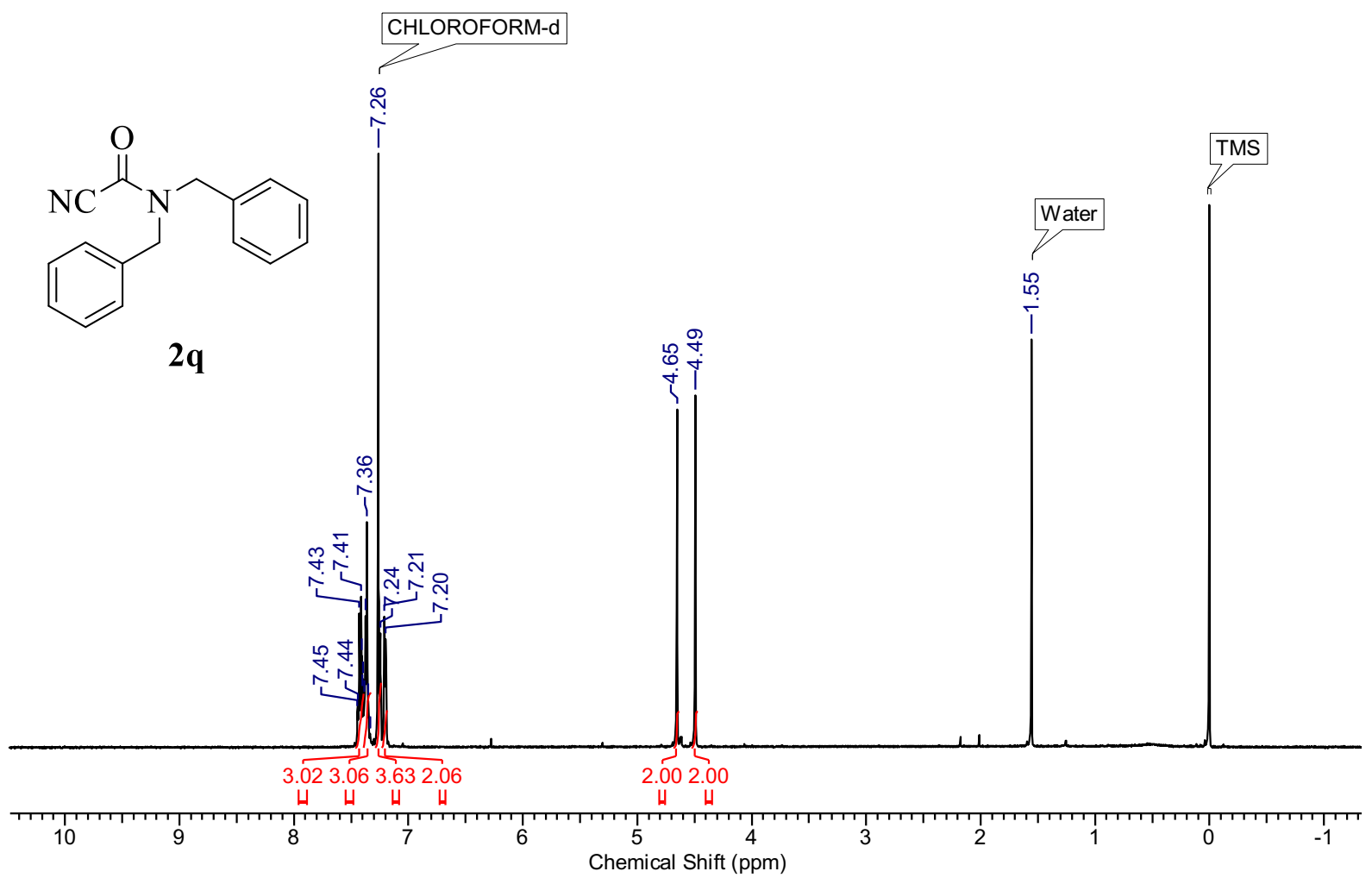

Figure S51. ${ }^{1} \mathrm{H}$ NMR spectrum of $2 \mathrm{q}$ in $\mathrm{CDCl}_{3}(500 \mathrm{MHz})$.

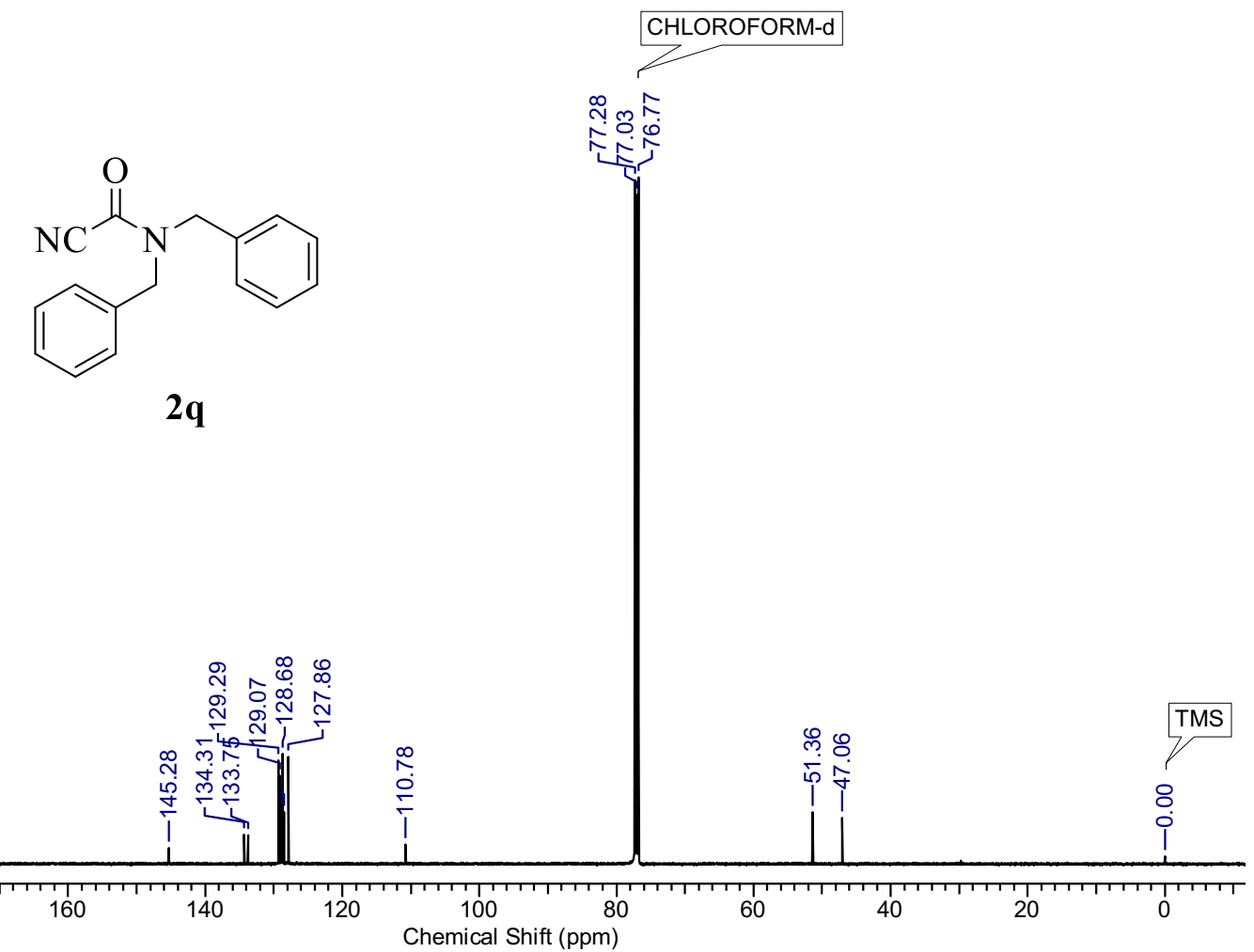

Figure S52. ${ }^{13} \mathrm{C}\left\{{ }^{1} \mathrm{H}\right\}$ NMR spectrum of $2 q$ in $\mathrm{CDCl}_{3}(125 \mathrm{MHz})$. 
<smiles>CN(Cc1ccccc1)C(=O)C#N</smiles>

2r

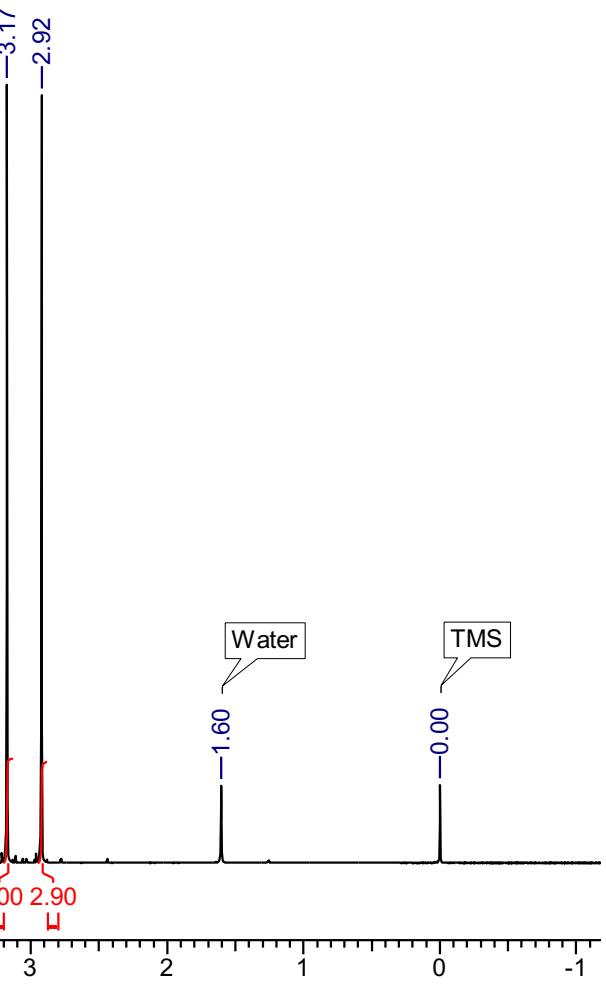

Figure S53. ${ }^{1} \mathrm{H}$ NMR spectrum of $2 \mathrm{r}$ in $\mathrm{CDCl}_{3}(500 \mathrm{MHz})$.

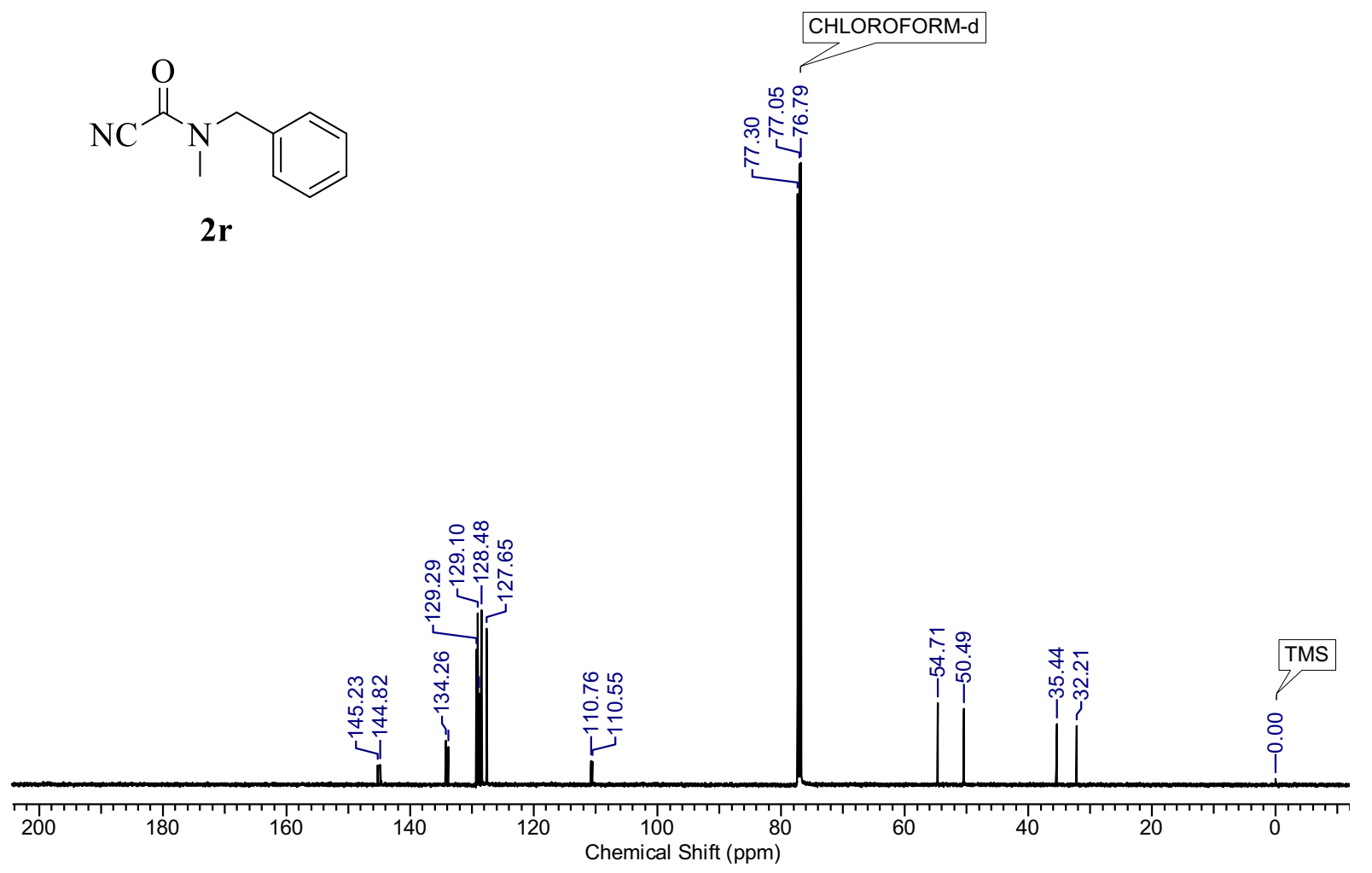

Figure S54. ${ }^{13} \mathrm{C}\left\{{ }^{1} \mathrm{H}\right\}$ NMR spectrum of $2 \mathrm{r}$ in $\mathrm{CDCl}_{3}(125 \mathrm{MHz})$. 


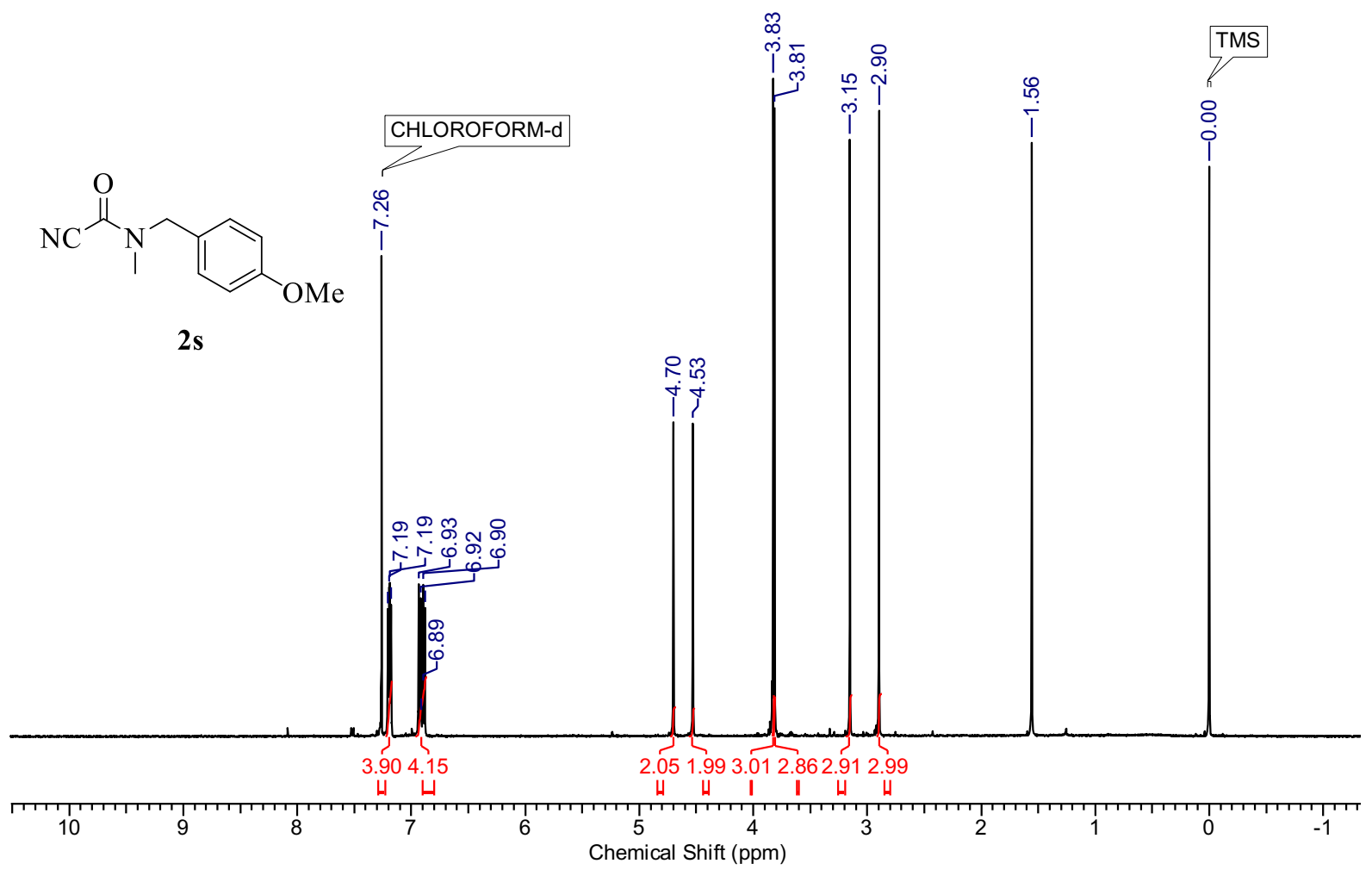

Figure S55. ${ }^{1} \mathrm{H}$ NMR spectrum of $2 \mathrm{~s}$ in $\mathrm{CDCl}_{3}(500 \mathrm{MHz})$.

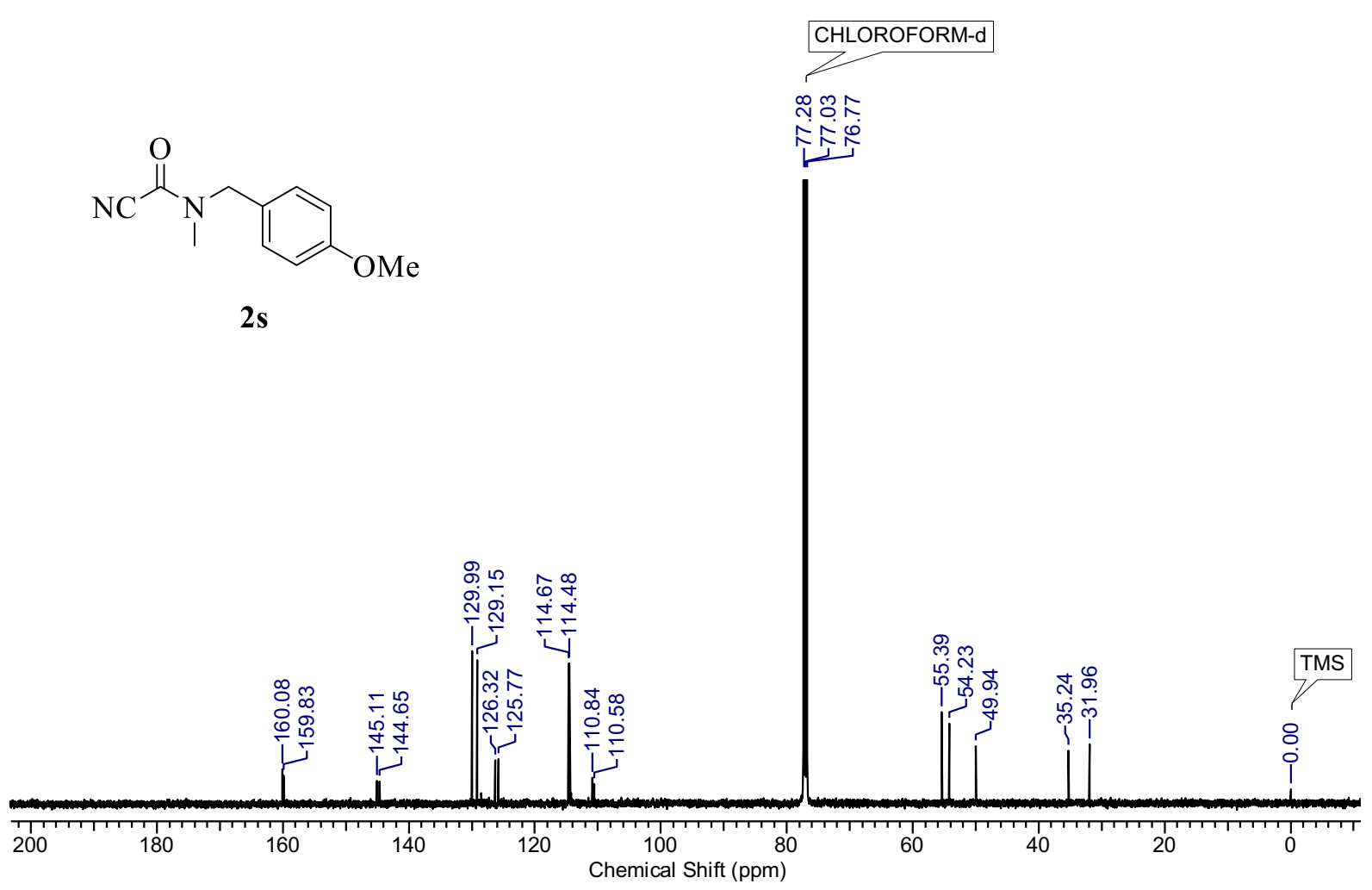

Figure S56. ${ }^{13} \mathrm{C}\left\{{ }^{1} \mathrm{H}\right\}$ NMR spectrum of $2 \mathbf{s}$ in $\mathrm{CDCl}_{3}(125 \mathrm{MHz})$. 
<smiles>CN(Cc1ccc(F)cc1)C(=O)C#N</smiles>

2t

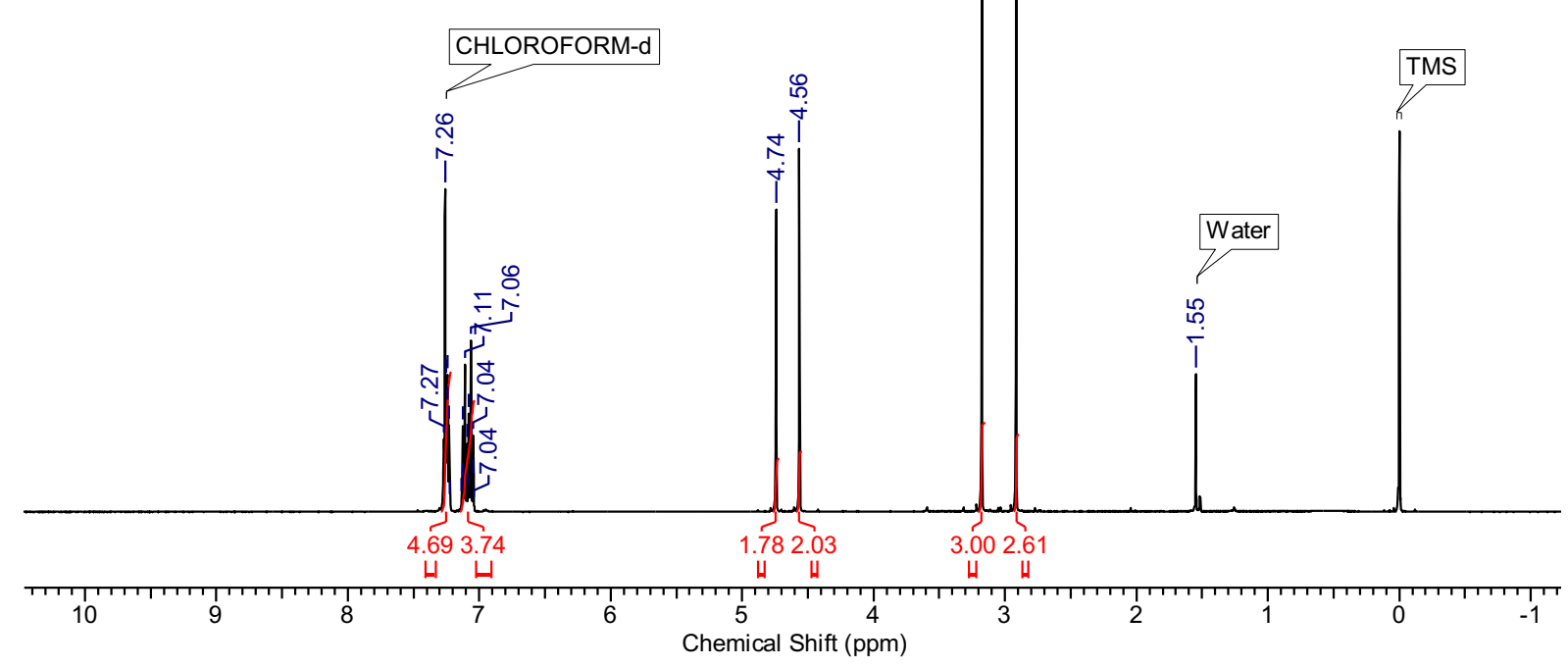

Figure S57. ${ }^{1} \mathrm{H}$ NMR spectrum of $2 \mathrm{t}$ in $\mathrm{CDCl}_{3}(500 \mathrm{MHz})$.

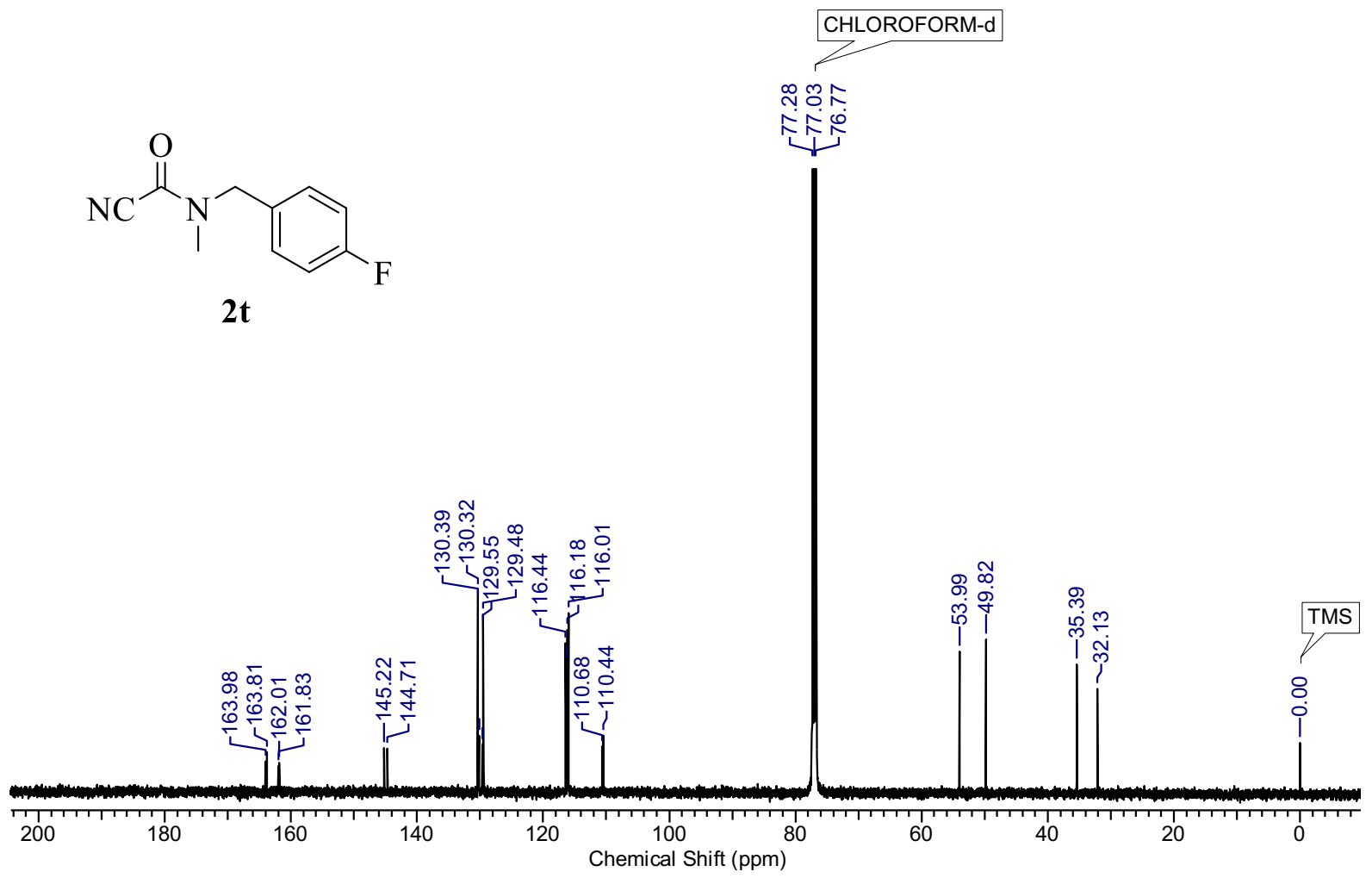

Figure S58. ${ }^{13} \mathrm{C}\left\{{ }^{1} \mathrm{H}\right\}$ NMR spectrum of $2 \mathrm{t}$ in $\mathrm{CDCl}_{3}(125 \mathrm{MHz})$. 


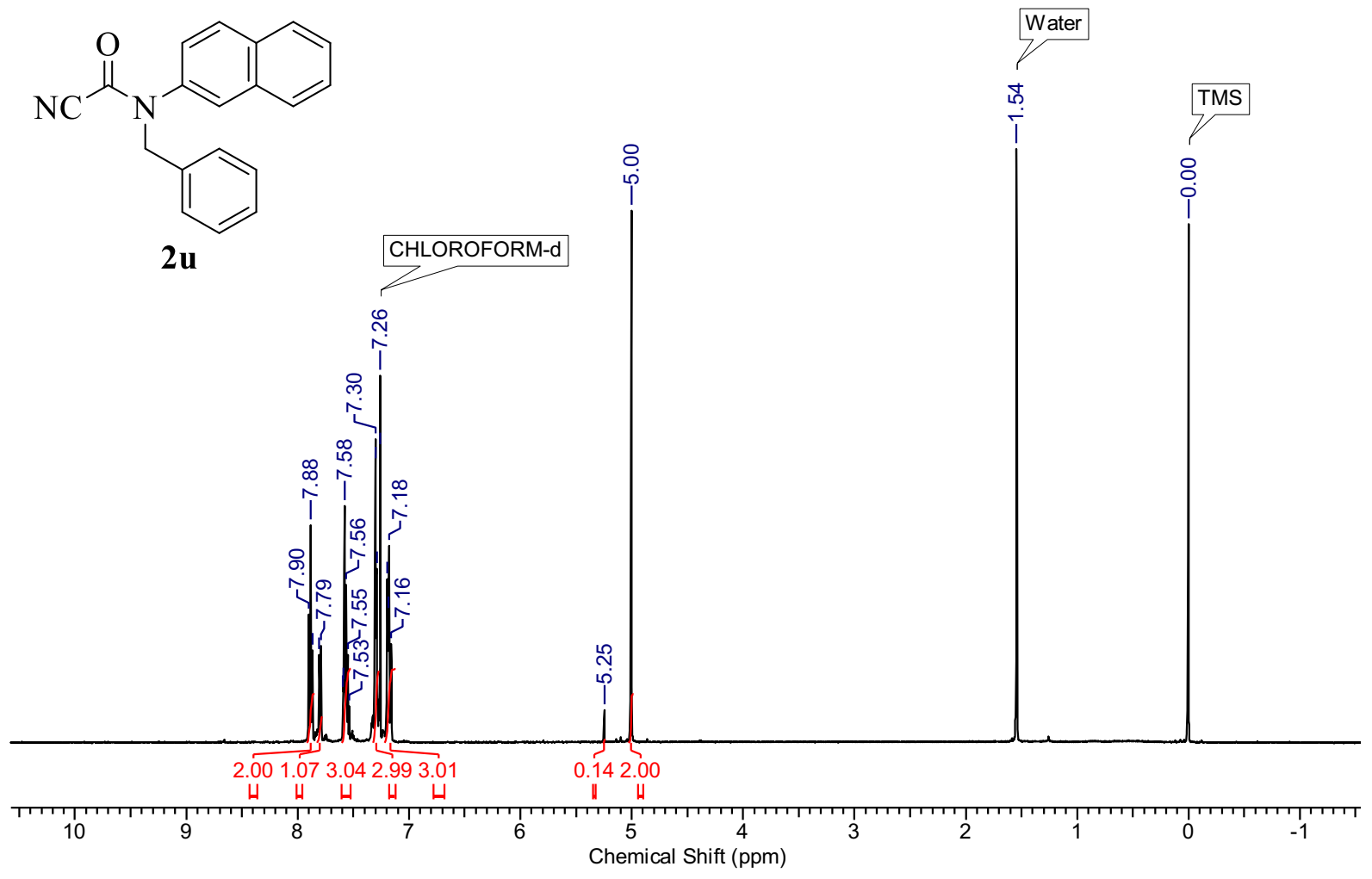

Figure S59. ${ }^{1} \mathrm{H}$ NMR spectrum of $2 \mathbf{u}$ in $\mathrm{CDCl}_{3}(500 \mathrm{MHz})$.

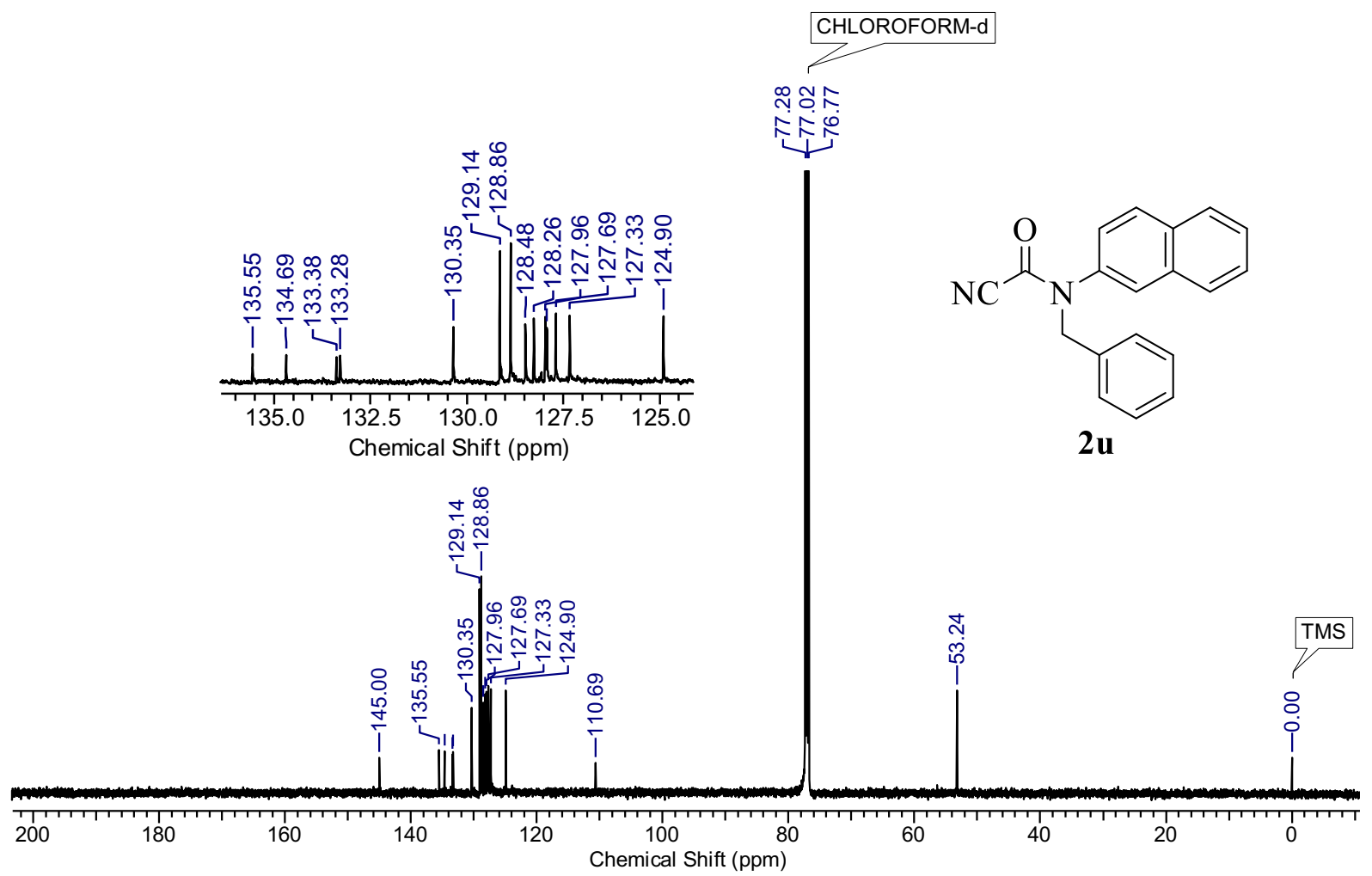

Figure S60. ${ }^{13} \mathrm{C}\left\{{ }^{1} \mathrm{H}\right\}$ NMR spectrum of $2 \mathbf{u}$ in $\mathrm{CDCl}_{3}(125 \mathrm{MHz})$. 


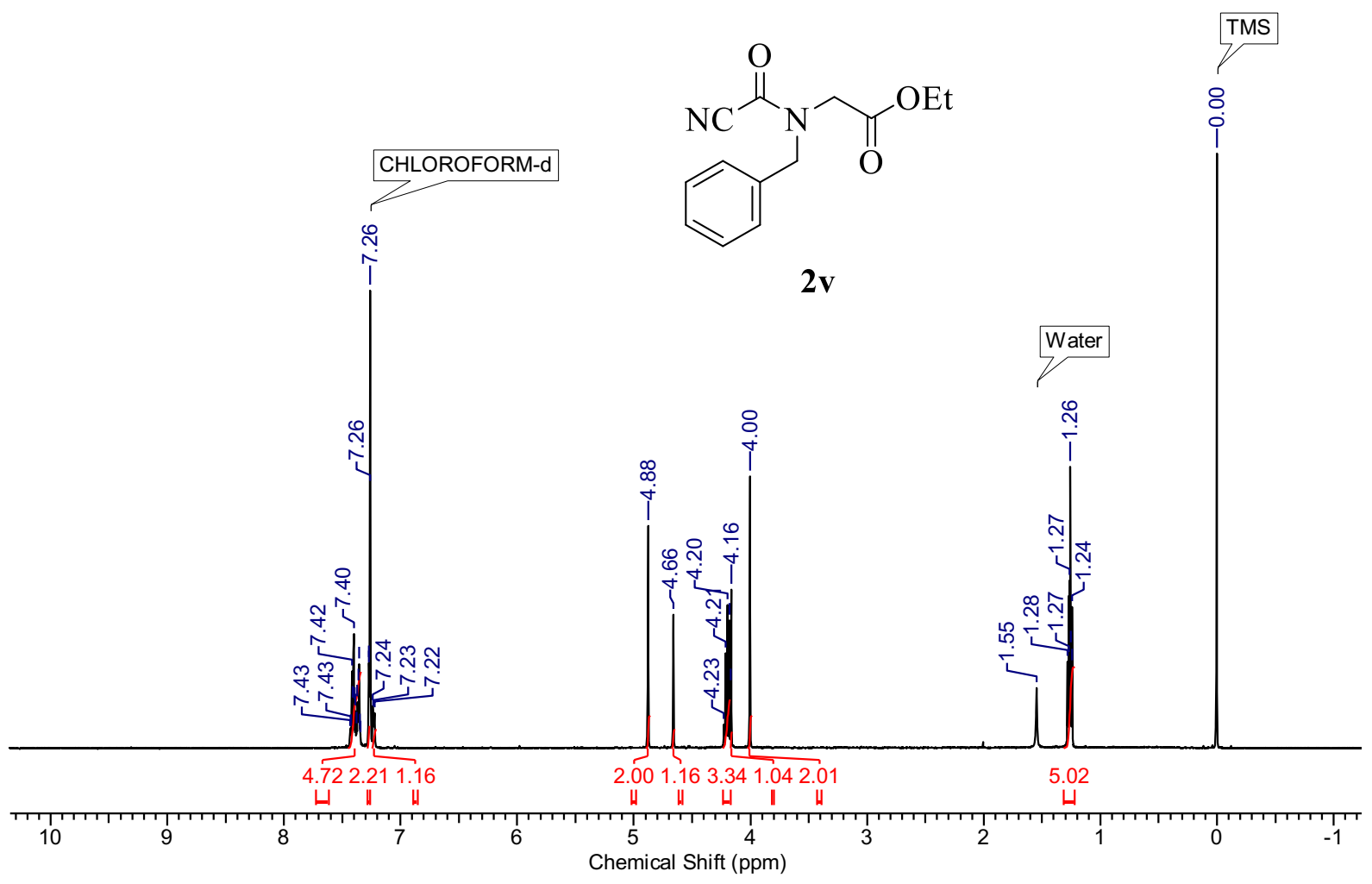

Figure S61. ${ }^{1} \mathrm{H}$ NMR spectrum of $2 \mathbf{v}$ in $\mathrm{CDCl}_{3}(500 \mathrm{MHz})$.<smiles>CCOC(=O)CN(Cc1ccccc1)C(=O)C#N</smiles>

\section{CHLOROFORM-d}

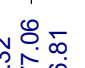

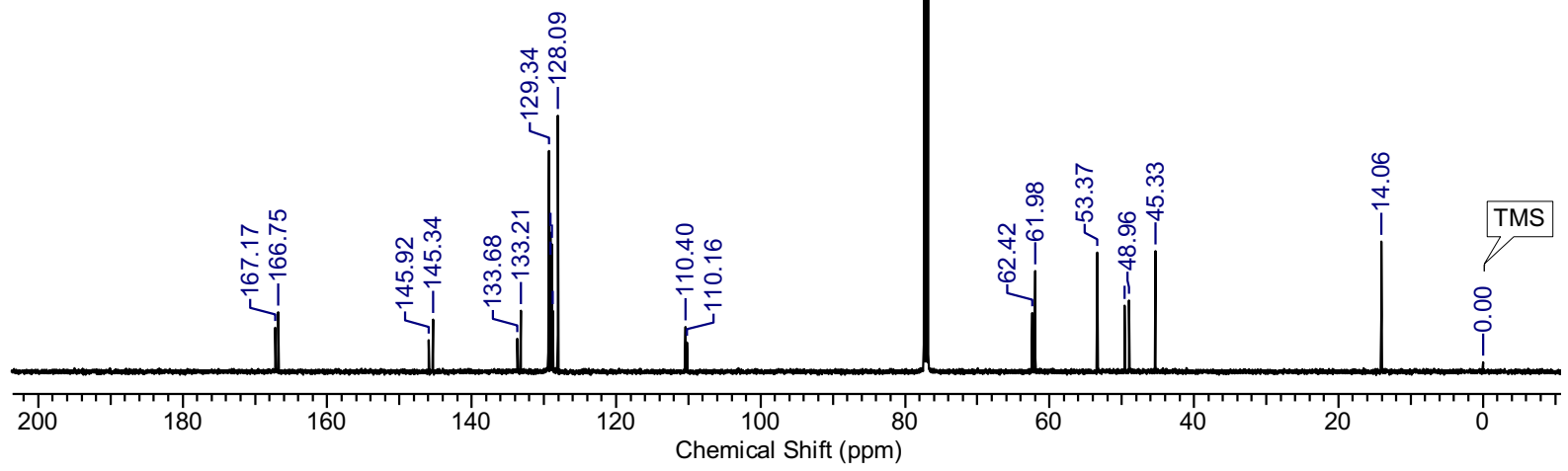

Figure S62. ${ }^{13} \mathrm{C}\left\{{ }^{1} \mathrm{H}\right\}$ NMR spectrum of $2 \mathbf{v}$ in $\mathrm{CDCl}_{3}(125 \mathrm{MHz})$. 


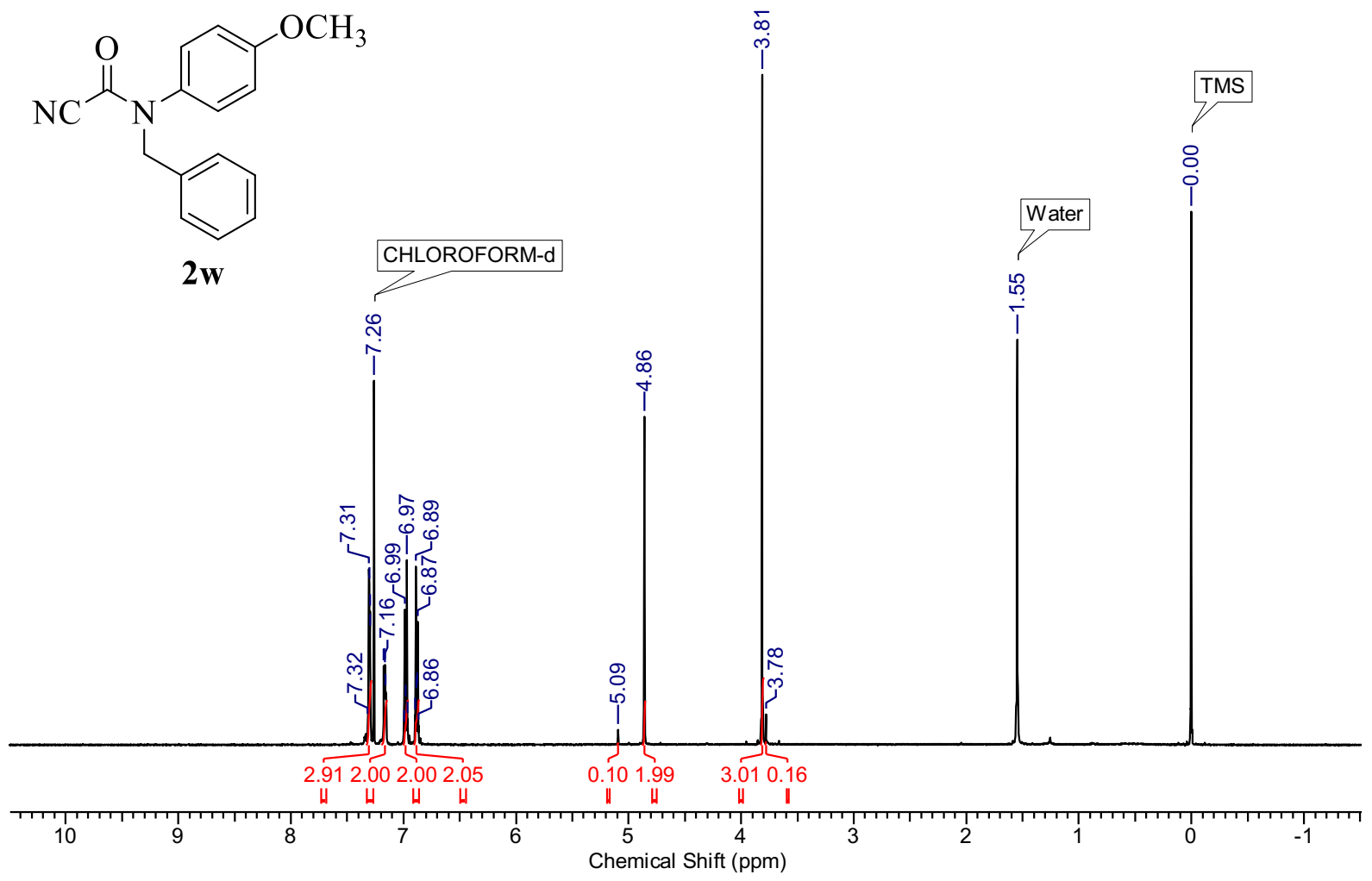

Figure S63. ${ }^{1} \mathrm{H}$ NMR spectrum of $2 \mathbf{w}$ in $\mathrm{CDCl}_{3}(500 \mathrm{MHz})$.

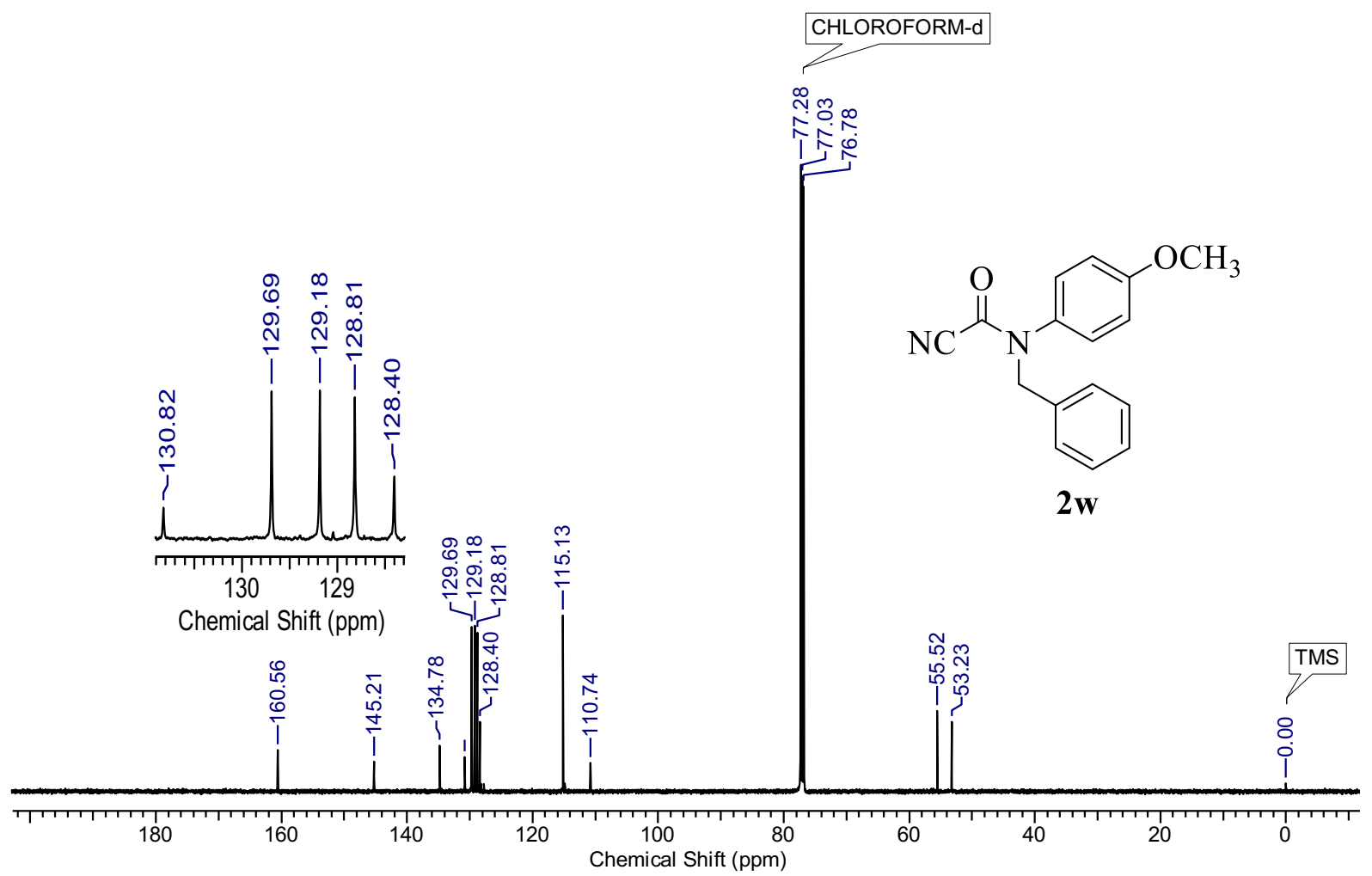

Figure S64. ${ }^{13} \mathrm{C}\left\{{ }^{1} \mathrm{H}\right\}$ NMR spectrum of $\mathbf{2 w}$ in $\mathrm{CDCl}_{3}(125 \mathrm{MHz})$. 


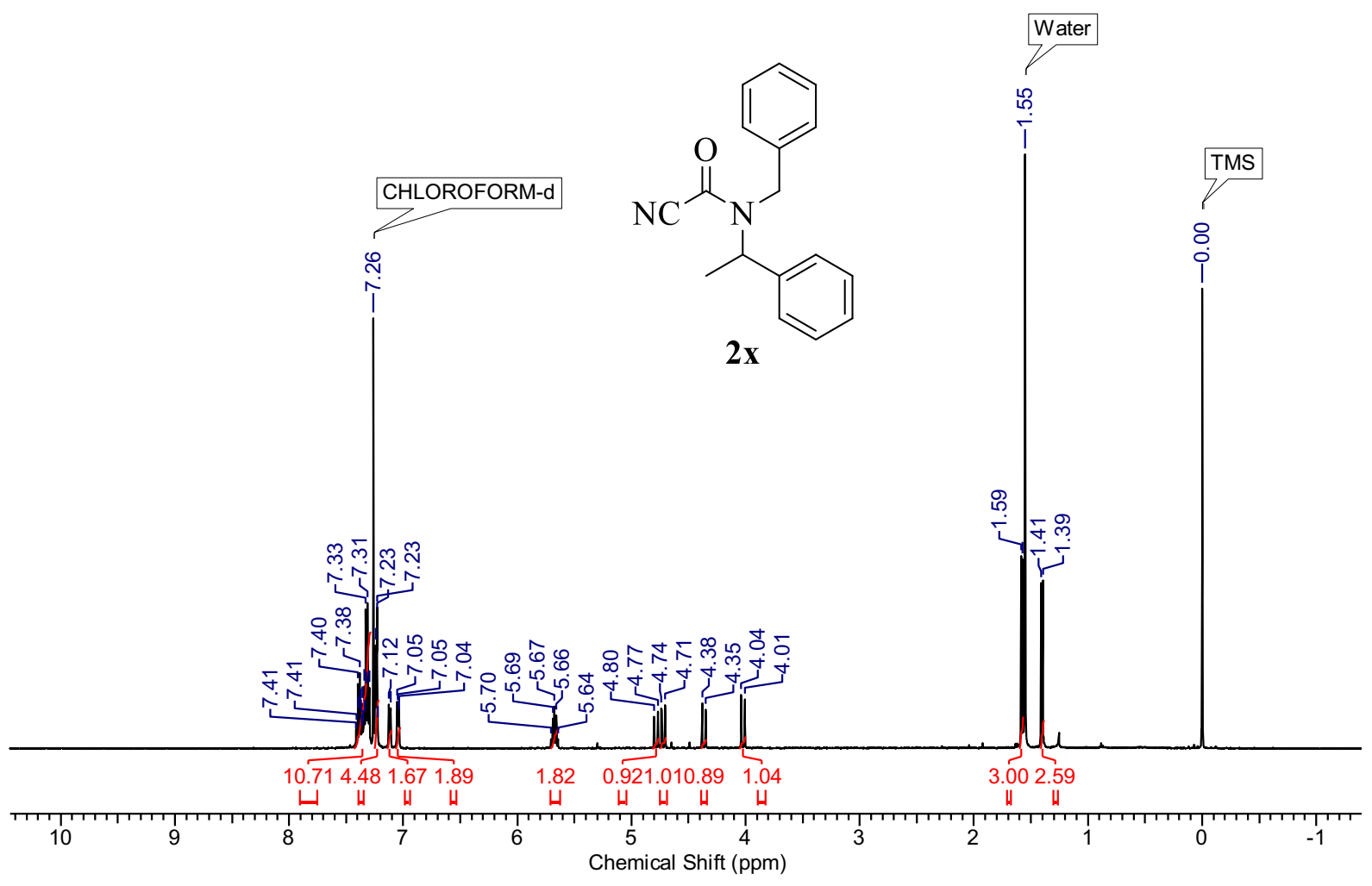

Figure S65. ${ }^{1} \mathrm{H}$ NMR spectrum of $2 \mathrm{x}$ in $\mathrm{CDCl}_{3}(500 \mathrm{MHz})$.

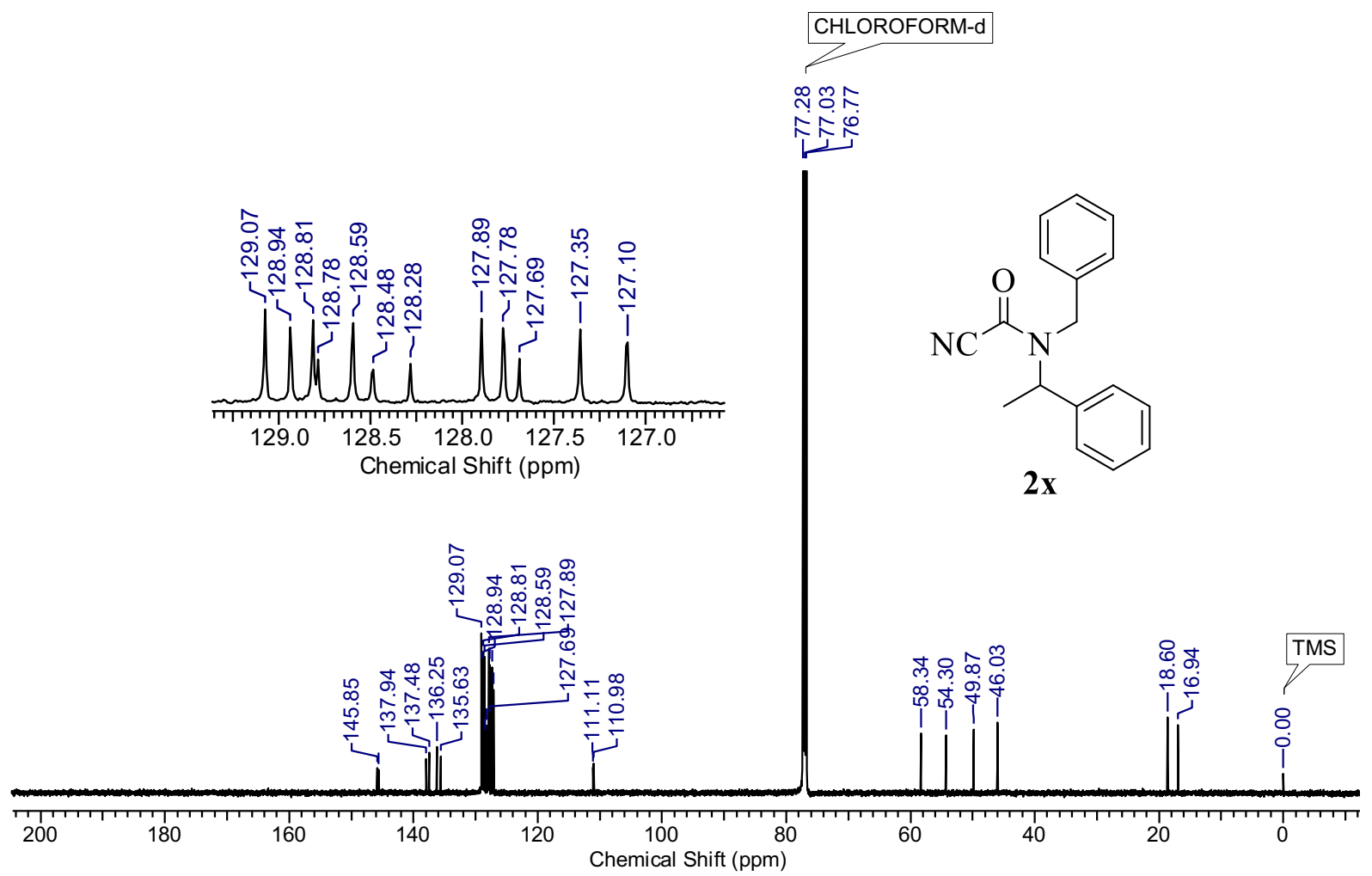

Figure S66. ${ }^{13} \mathrm{C}\left\{{ }^{1} \mathrm{H}\right\}$ NMR spectrum of $2 \mathbf{x}$ in $\mathrm{CDCl}_{3}(125 \mathrm{MHz})$. 


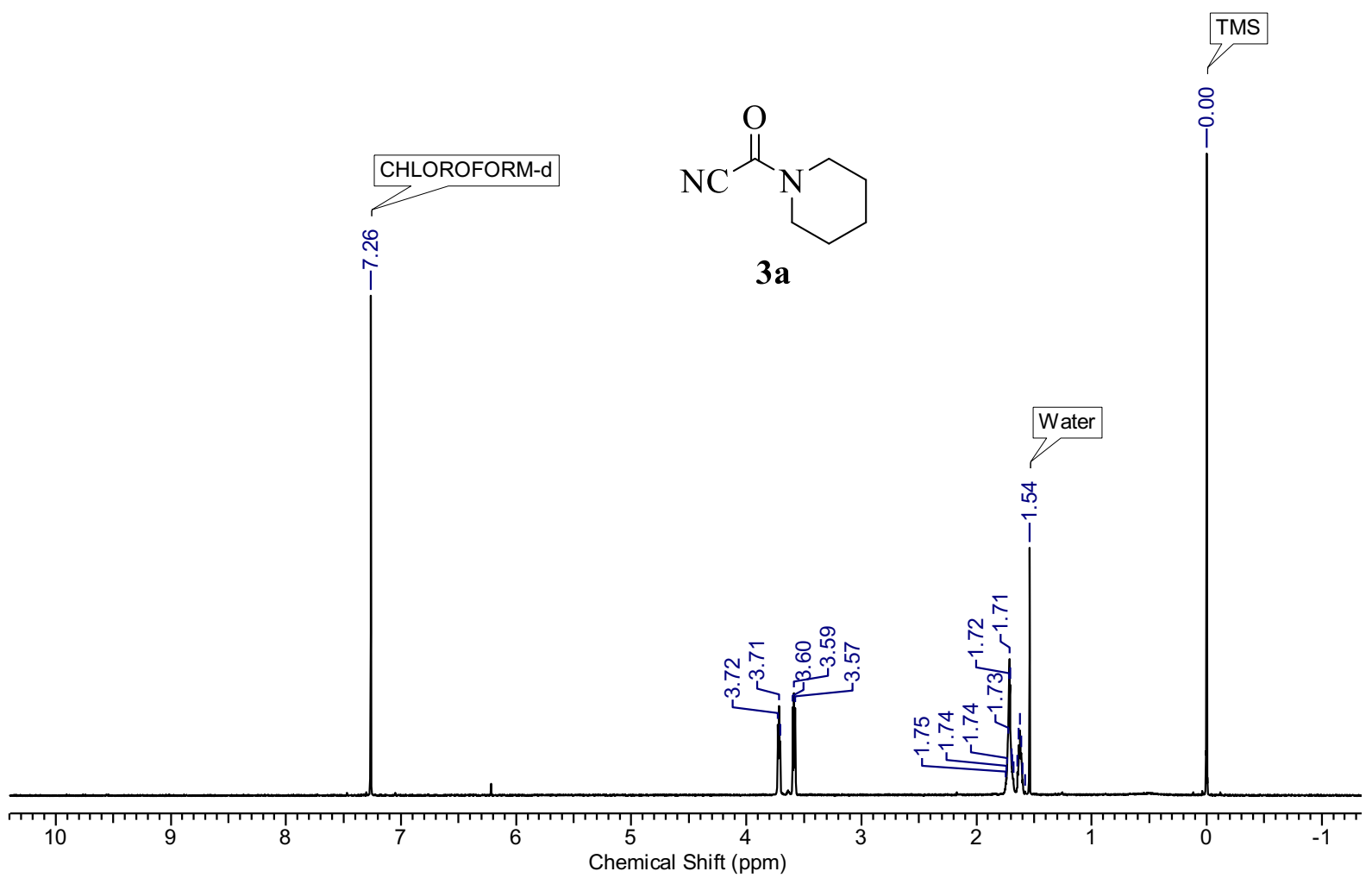

Figure S67. ${ }^{1} \mathrm{H}$ NMR spectrum of $3 \mathbf{a}$ in $\mathrm{CDCl}_{3}(500 \mathrm{MHz})$.<smiles>N#CC(=O)N1CCCCC1</smiles>

3a

Figure S68. ${ }^{13} \mathrm{C}\left\{{ }^{1} \mathrm{H}\right\}$ NMR spectrum of $\mathbf{3 a}$ in $\mathrm{CDCl}_{3}(125 \mathrm{MHz})$. 


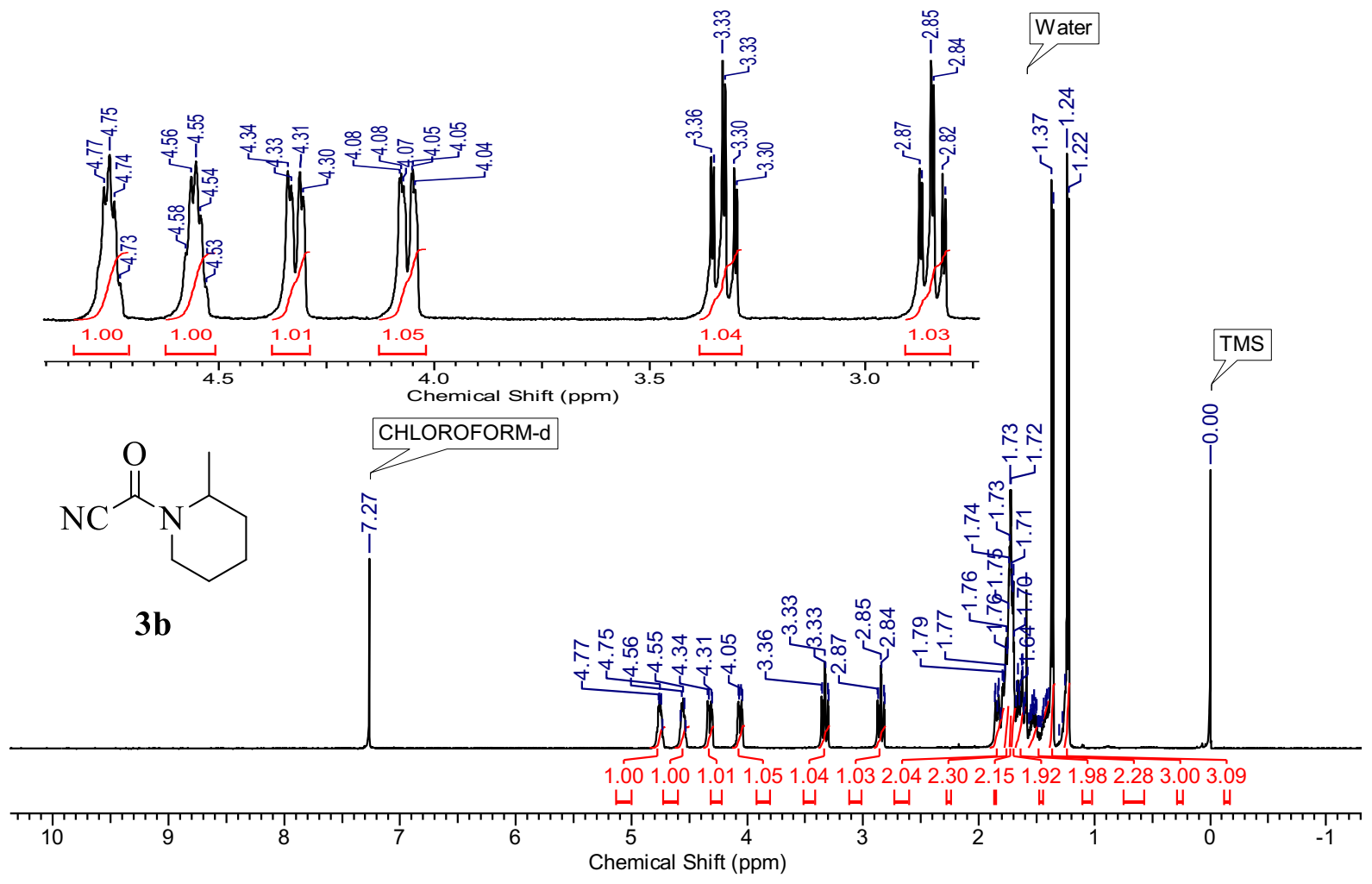

Figure S69. ${ }^{1} \mathrm{H}$ NMR spectrum of $\mathbf{3 b}$ in $\mathrm{CDCl}_{3}(500 \mathrm{MHz})$.

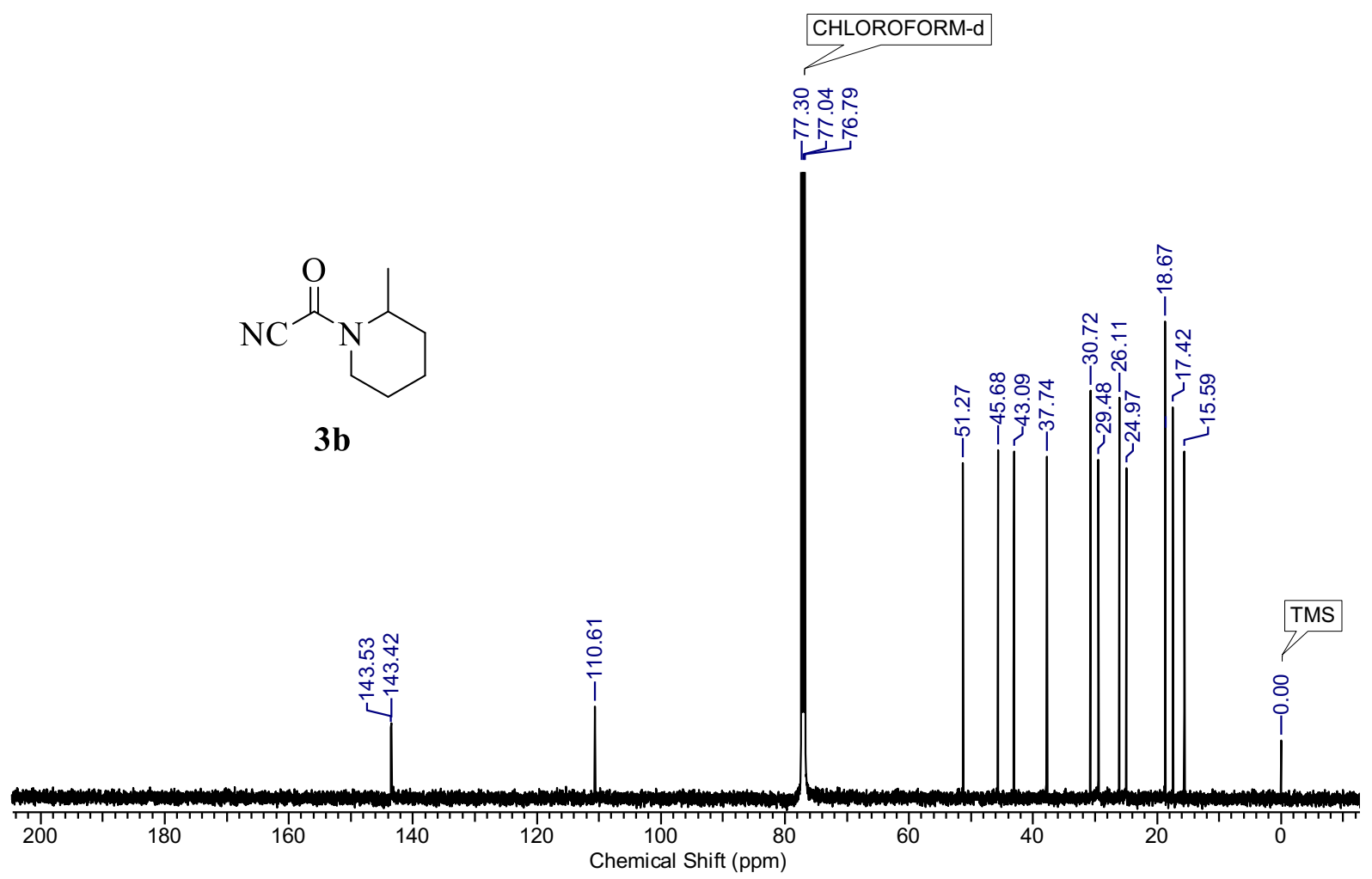

Figure S70. ${ }^{13} \mathrm{C}\left\{{ }^{1} \mathrm{H}\right\}$ NMR spectrum of $\mathbf{3 b}$ in $\mathrm{CDCl}_{3}(125 \mathrm{MHz})$. 


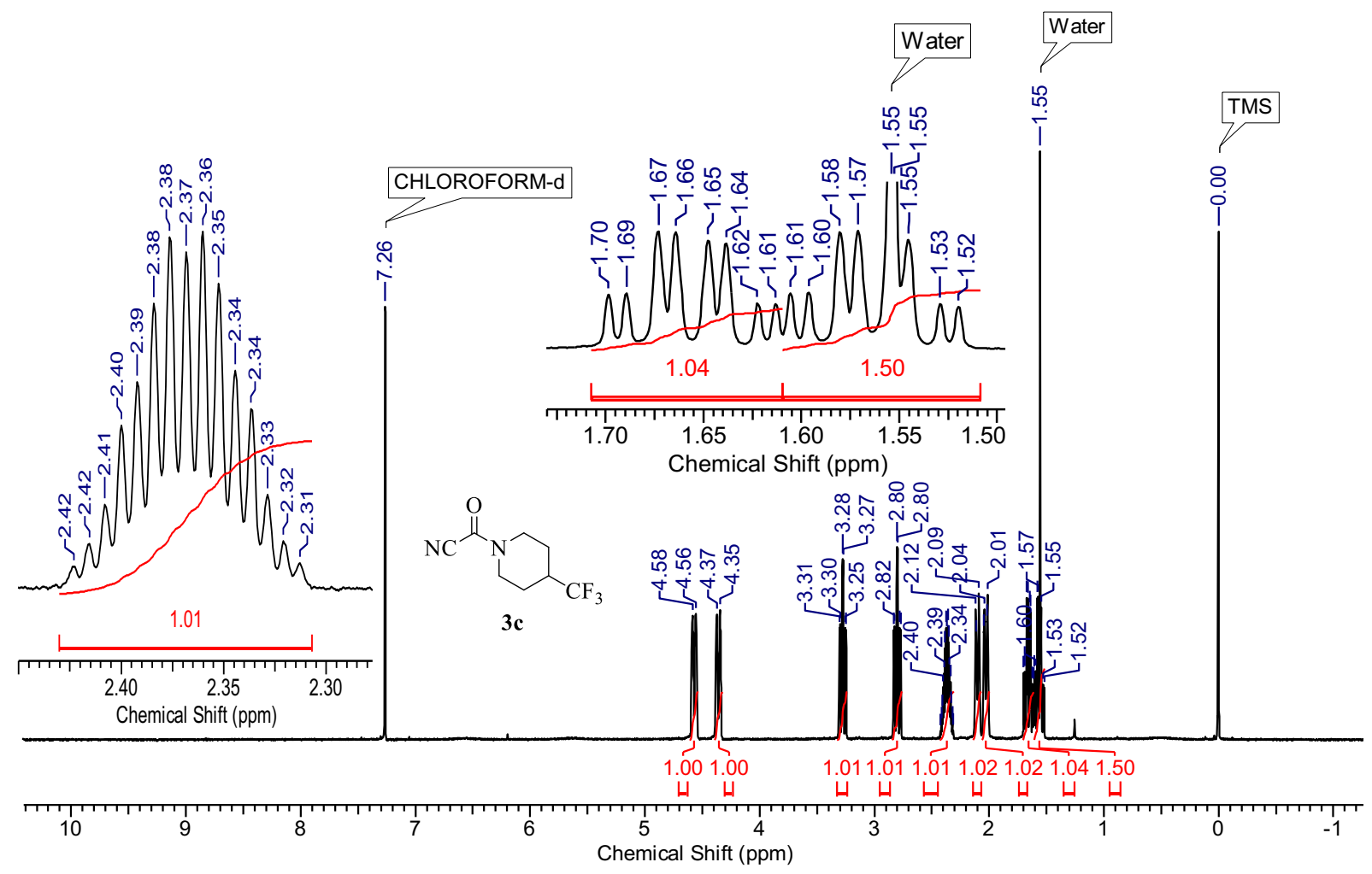

Figure S71. ${ }^{1} \mathrm{H}$ NMR spectrum of $3 \mathrm{c}$ in $\mathrm{CDCl}_{3}(500 \mathrm{MHz})$.

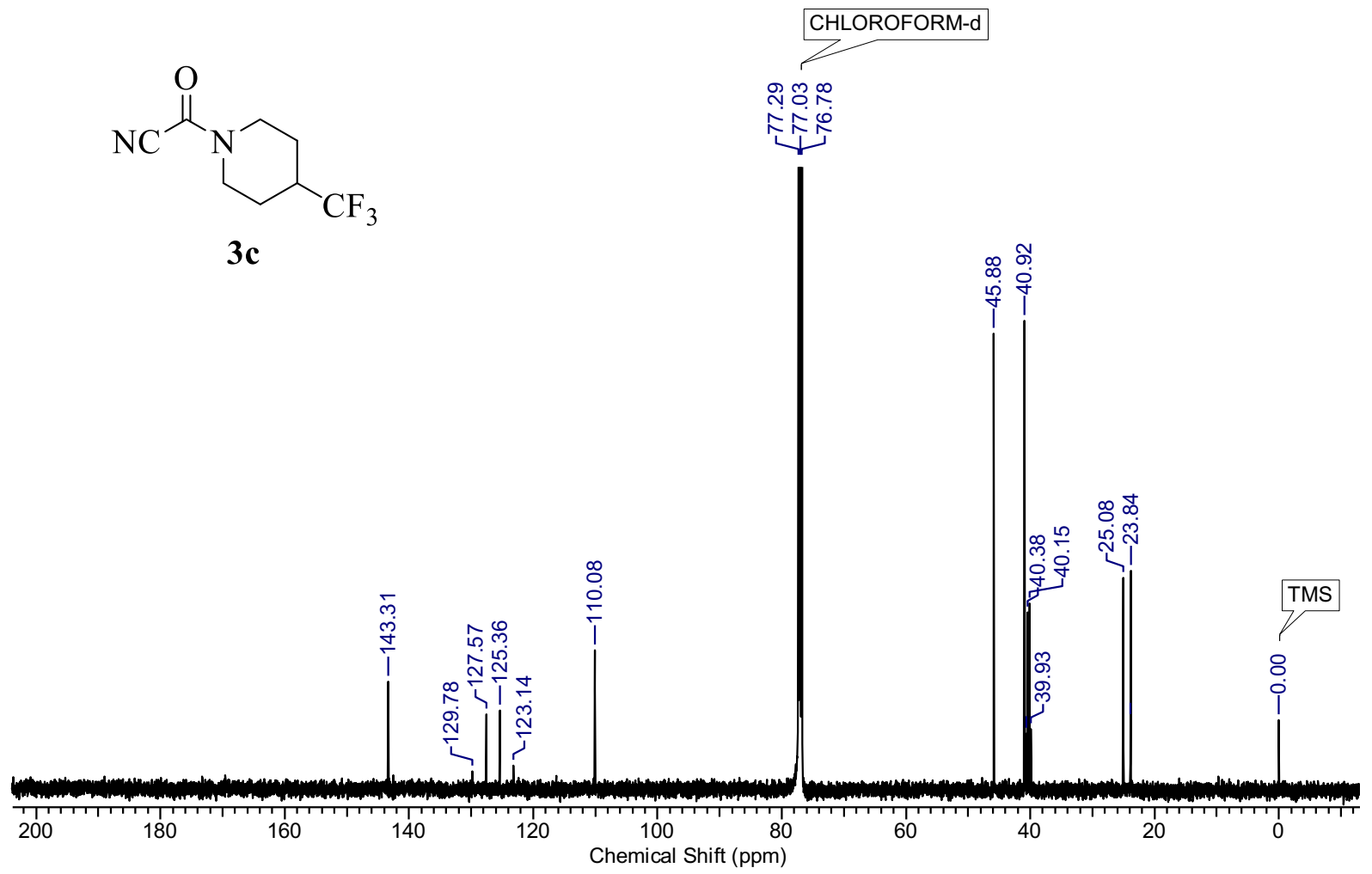

Figure S72. ${ }^{13} \mathrm{C}\left\{{ }^{1} \mathrm{H}\right\}$ NMR spectrum of $3 \mathrm{c}$ in $\mathrm{CDCl}_{3}(125 \mathrm{MHz})$. 


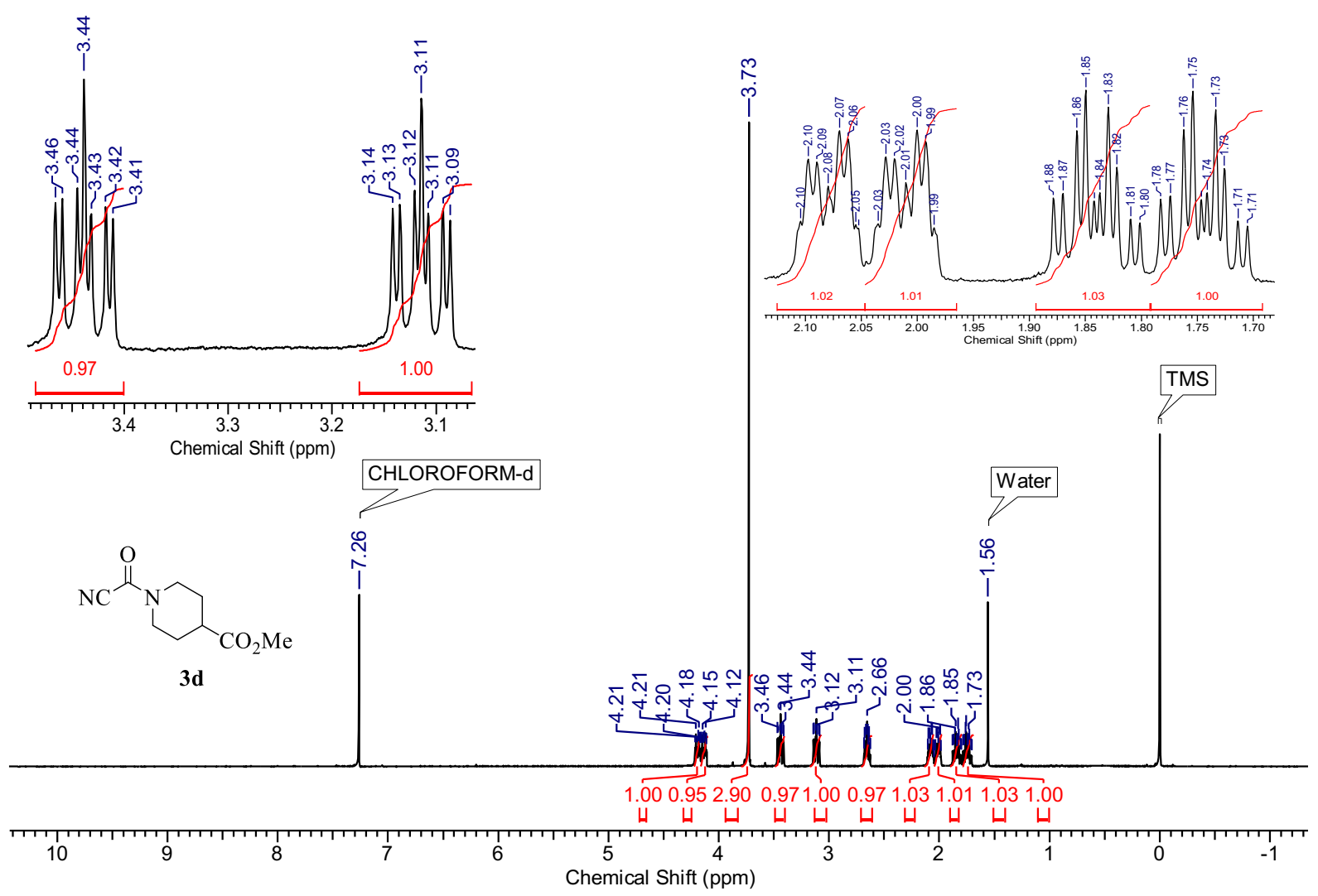

Figure S73. ${ }^{1} \mathrm{H}$ NMR spectrum of $\mathbf{3 d}$ in $\mathrm{CDCl}_{3}(500 \mathrm{MHz})$.
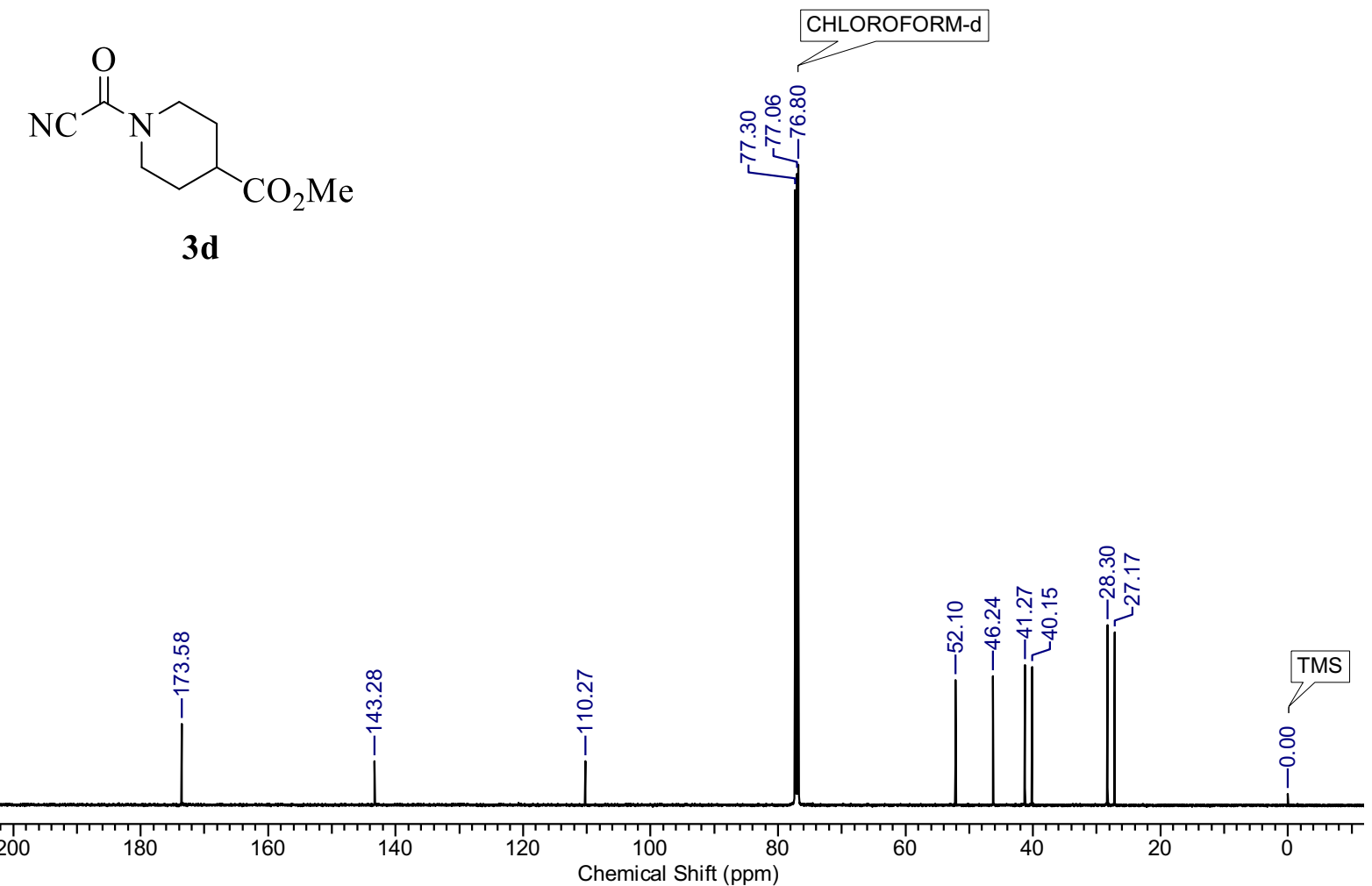

Figure S74. ${ }^{13} \mathrm{C}\left\{{ }^{1} \mathrm{H}\right\}$ NMR spectrum of $\mathbf{3 d}$ in $\mathrm{CDCl}_{3}(125 \mathrm{MHz})$. 


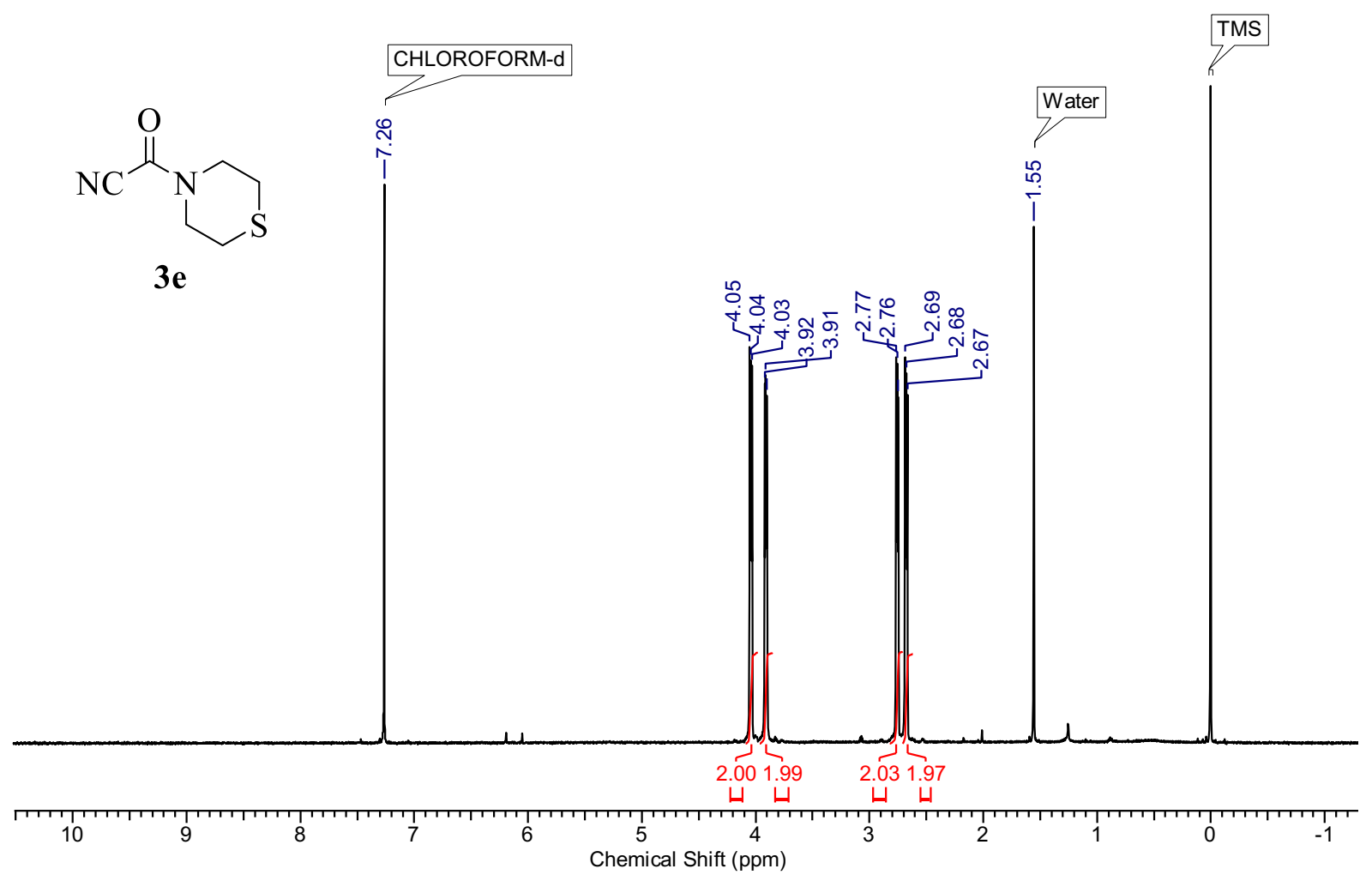

Figure S75. ${ }^{1} \mathrm{H}$ NMR spectrum of $3 \mathbf{e}$ in $\mathrm{CDCl}_{3}(500 \mathrm{MHz})$.

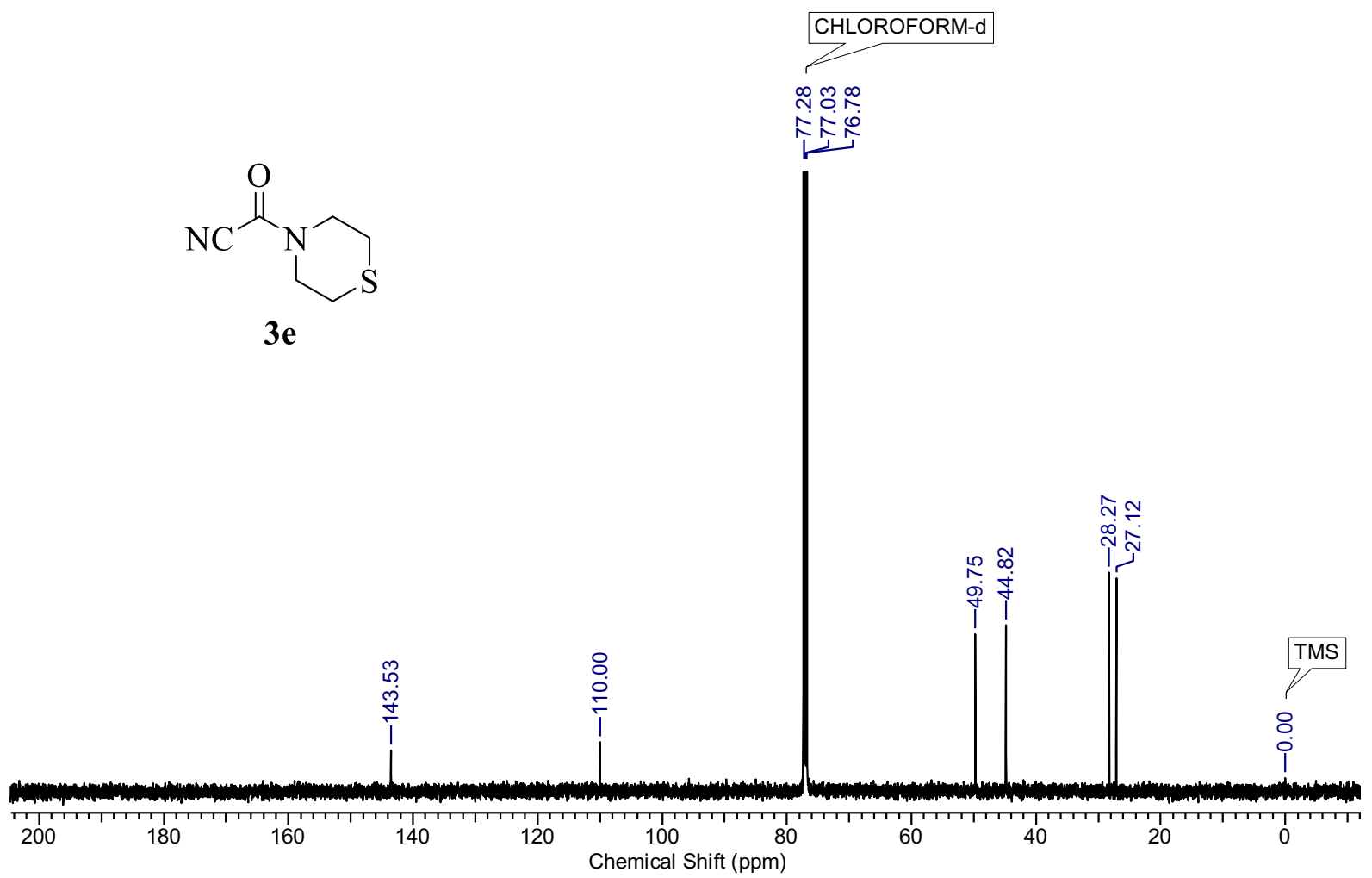

Figure S76. ${ }^{13} \mathrm{C}\left\{{ }^{1} \mathrm{H}\right\}$ NMR spectrum of $3 \mathrm{e}$ in $\mathrm{CDCl}_{3}(125 \mathrm{MHz})$. 

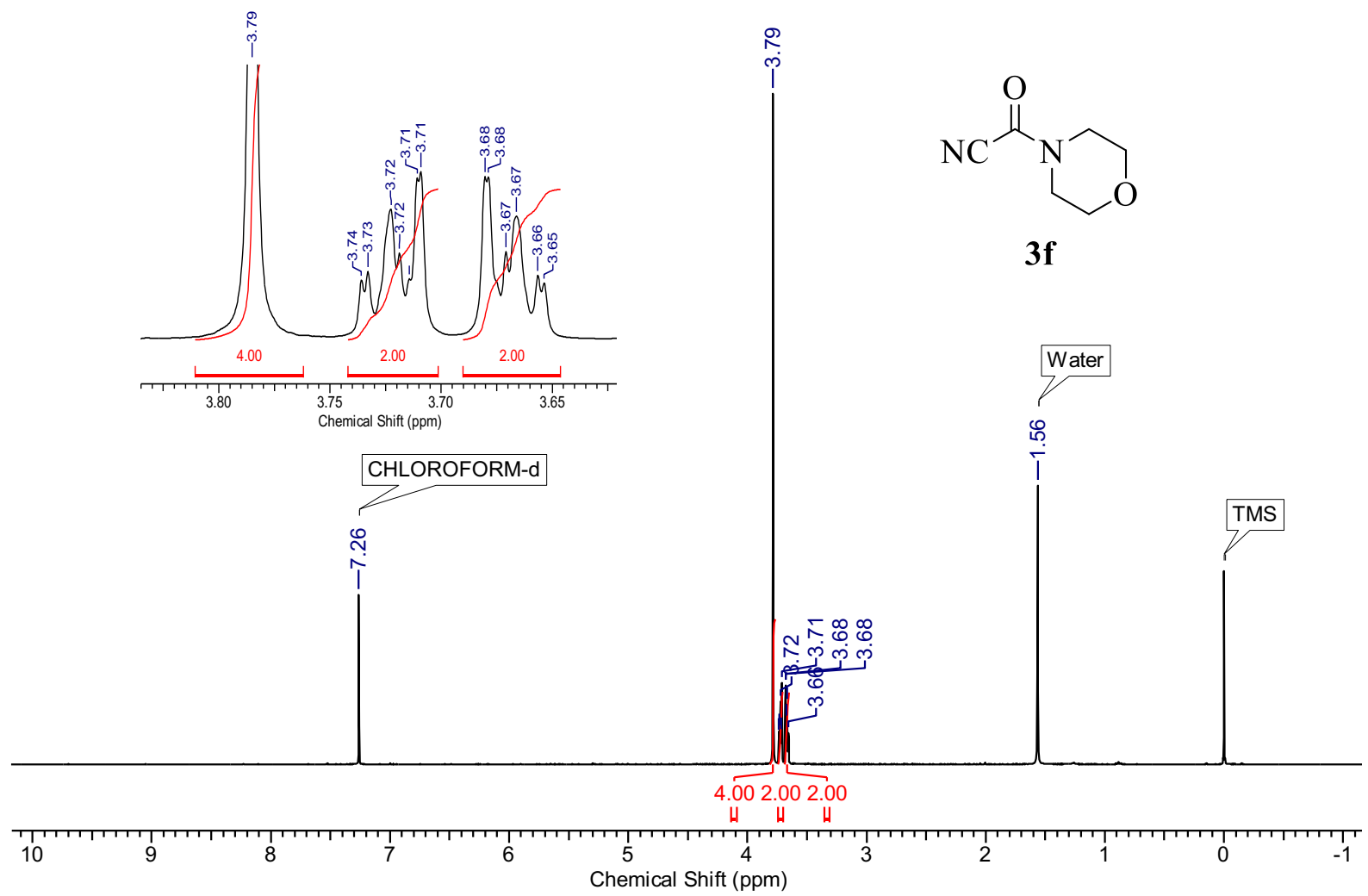

Figure S77. ${ }^{1} \mathrm{H}$ NMR spectrum of $\mathbf{3 f}$ in $\mathrm{CDCl}_{3}(400 \mathrm{MHz})$.

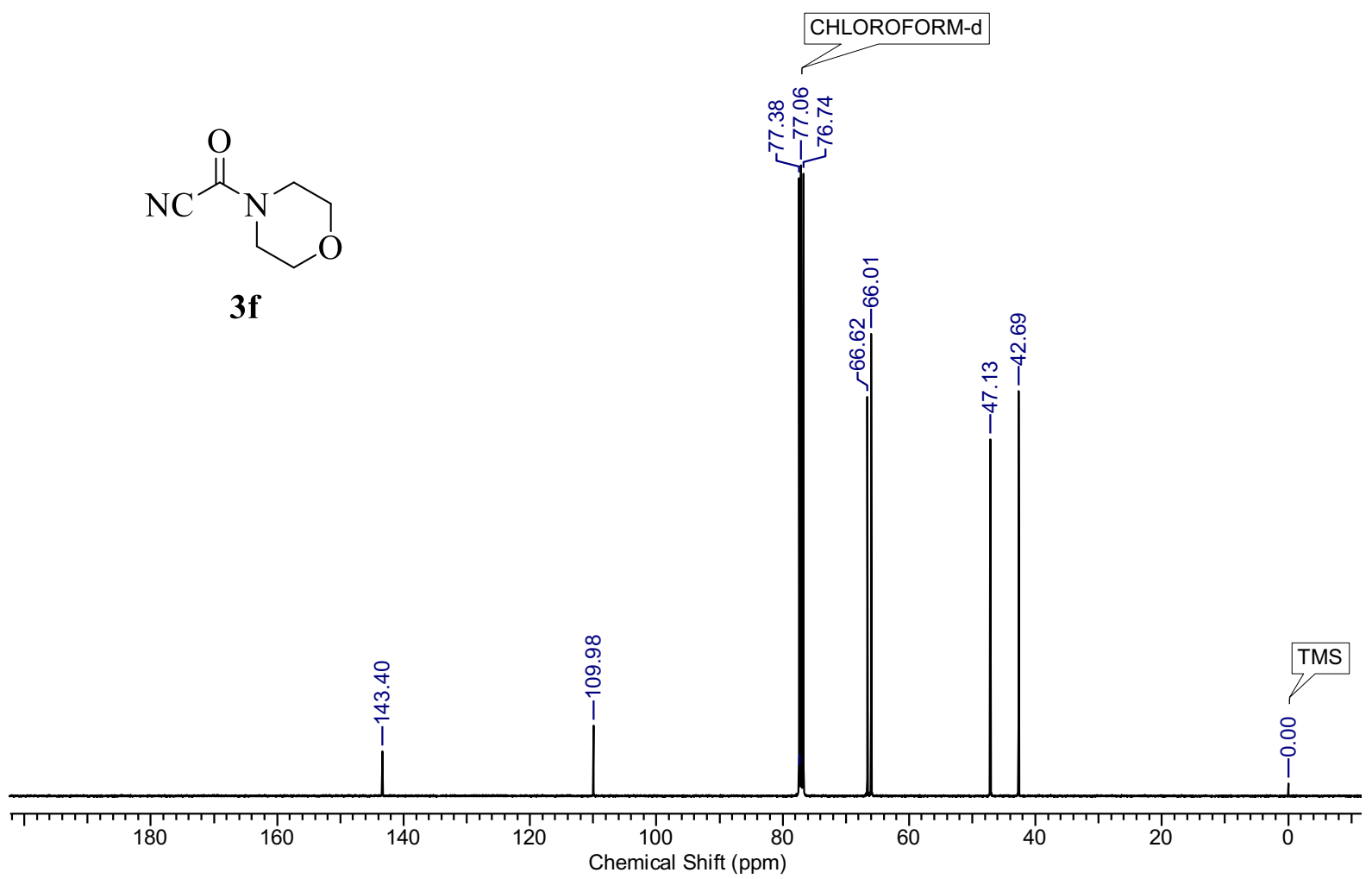

Figure S78. ${ }^{13} \mathrm{C}\left\{{ }^{1} \mathrm{H}\right\}$ NMR spectrum of $\mathbf{3 f}$ in $\mathrm{CDCl}_{3}(100 \mathrm{MHz})$. 


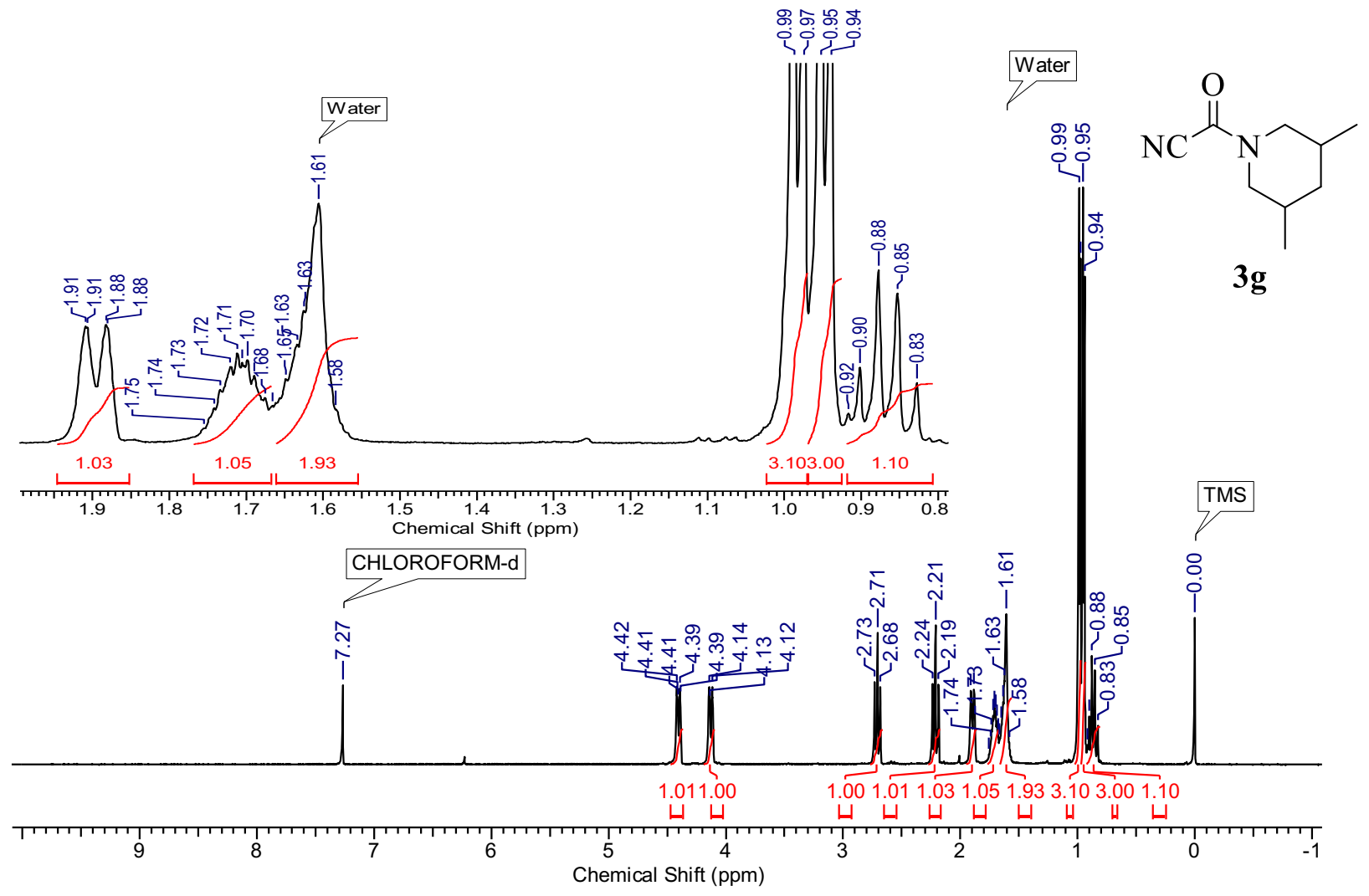

Figure S79. ${ }^{1} \mathrm{H}$ NMR spectrum of $\mathbf{3 g}$ in $\mathrm{CDCl}_{3}(500 \mathrm{MHz})$.

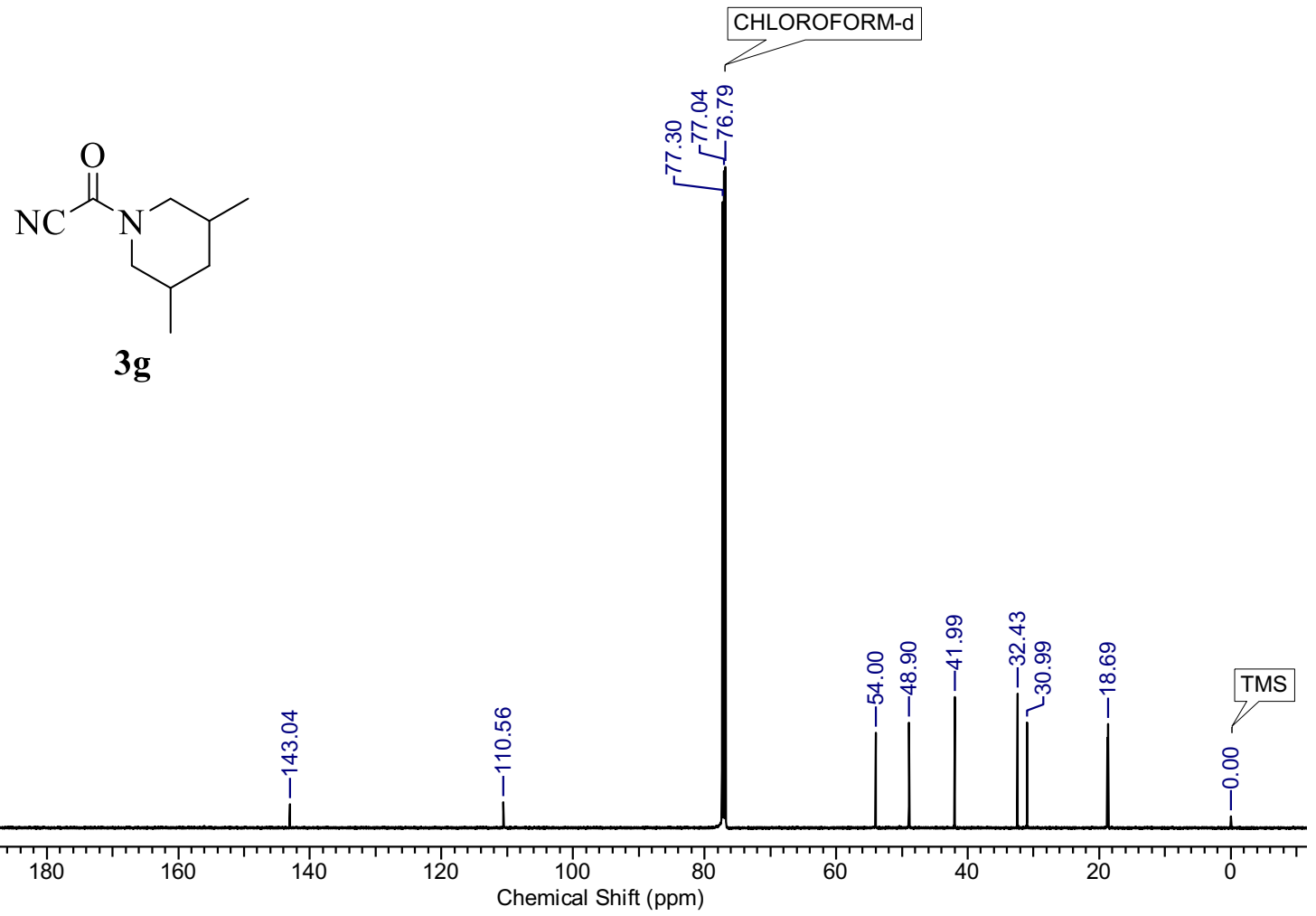

Figure S80. ${ }^{13} \mathrm{C}\left\{{ }^{1} \mathrm{H}\right\}$ NMR spectrum of $\mathbf{3 g}$ in $\mathrm{CDCl}_{3}(125 \mathrm{MHz})$. 


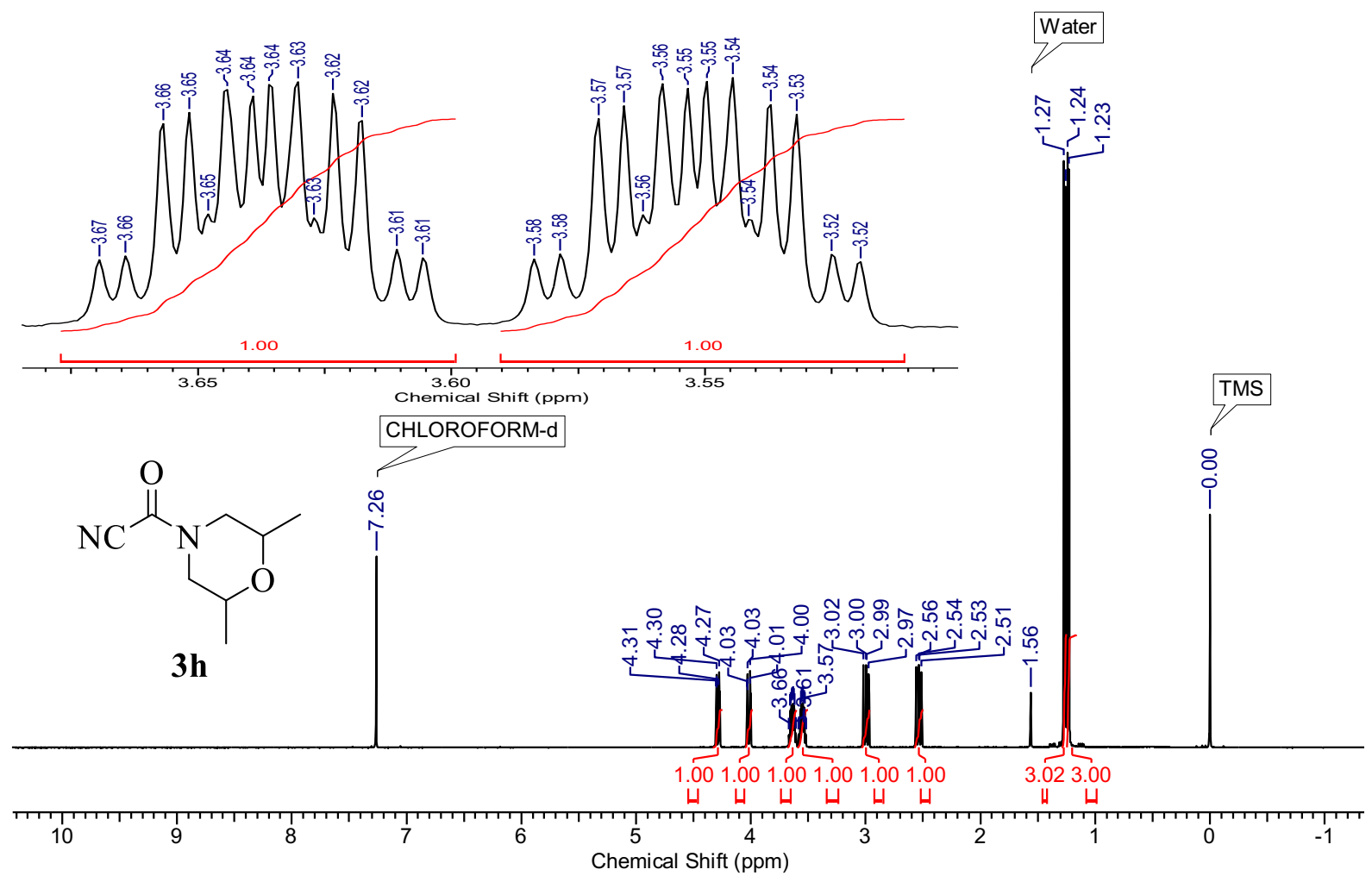

Figure S81. ${ }^{1} \mathrm{H}$ NMR spectrum of $\mathbf{3 h}$ in $\mathrm{CDCl}_{3}(500 \mathrm{MHz})$.

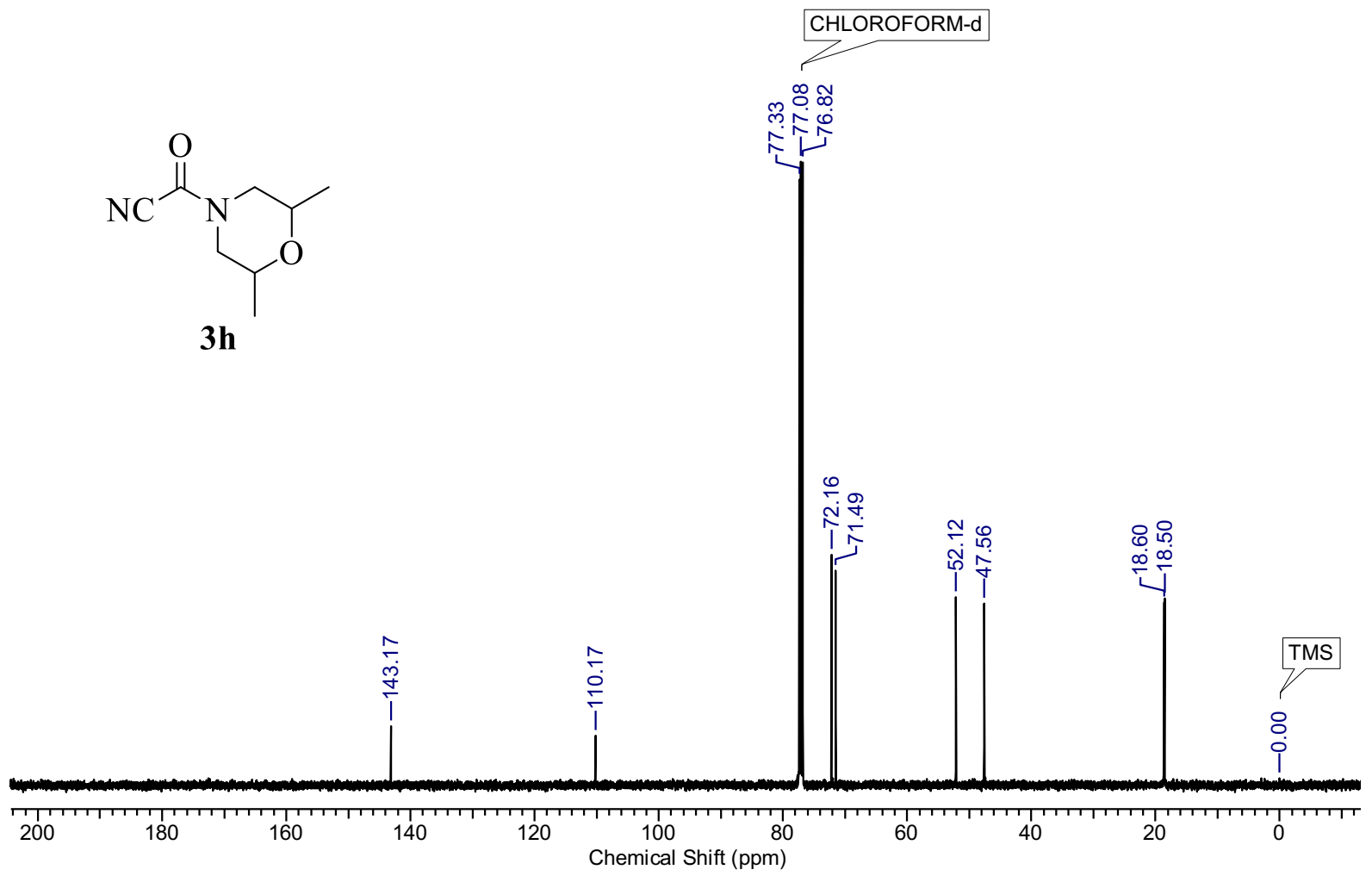

Figure S82. ${ }^{13} \mathrm{C}\left\{{ }^{1} \mathrm{H}\right\}$ NMR spectrum of $3 \mathbf{h}$ in $\mathrm{CDCl}_{3}(125 \mathrm{MHz})$. 


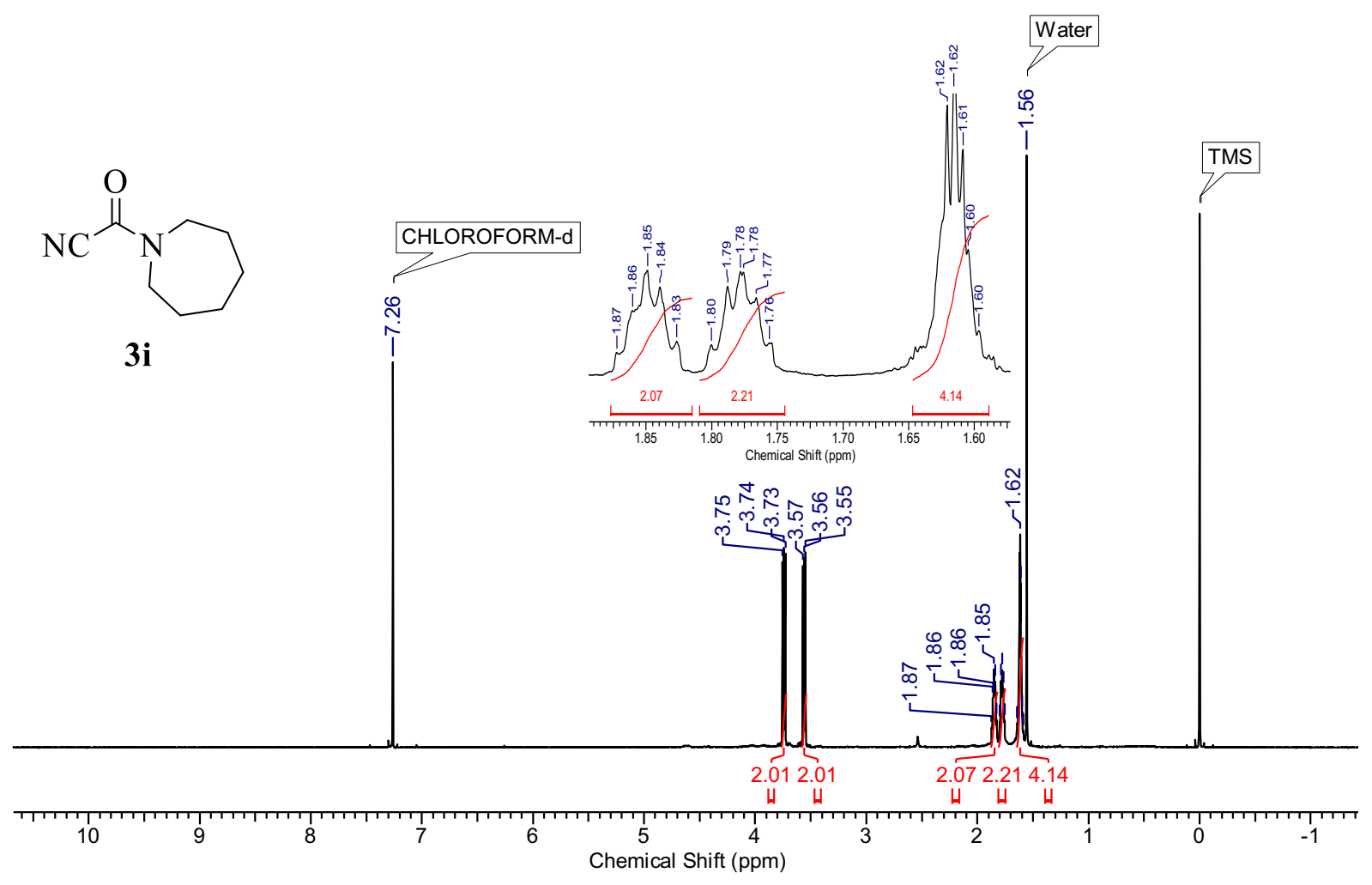

Figure S83. ${ }^{1} \mathrm{H}$ NMR spectrum of $3 \mathbf{i}$ in $\mathrm{CDCl}_{3}(500 \mathrm{MHz})$.
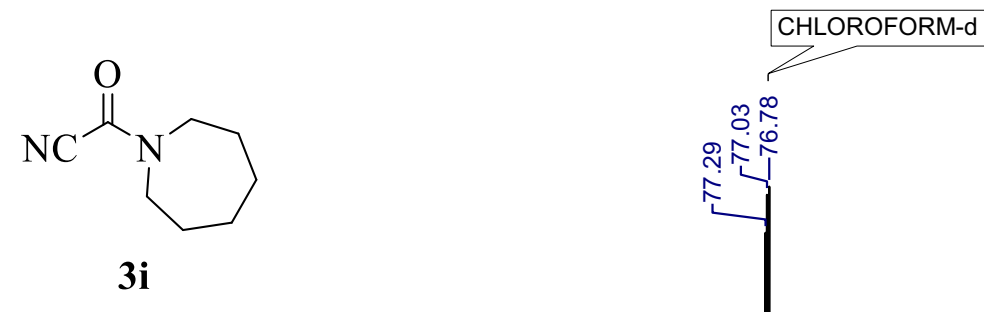

3i

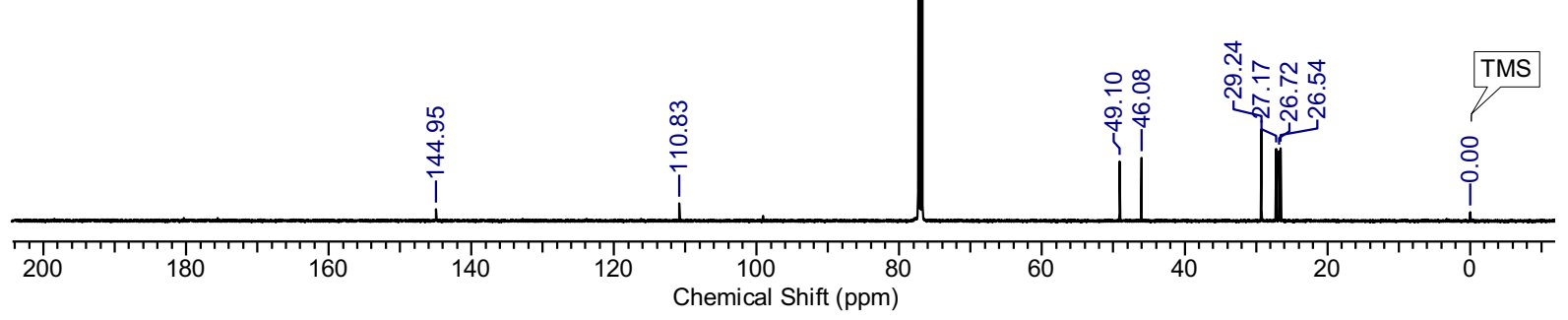

Figure S84. ${ }^{13} \mathrm{C}\left\{{ }^{1} \mathrm{H}\right\}$ NMR spectrum of $3 \mathrm{i}$ in $\mathrm{CDCl}_{3}(125 \mathrm{MHz})$. 


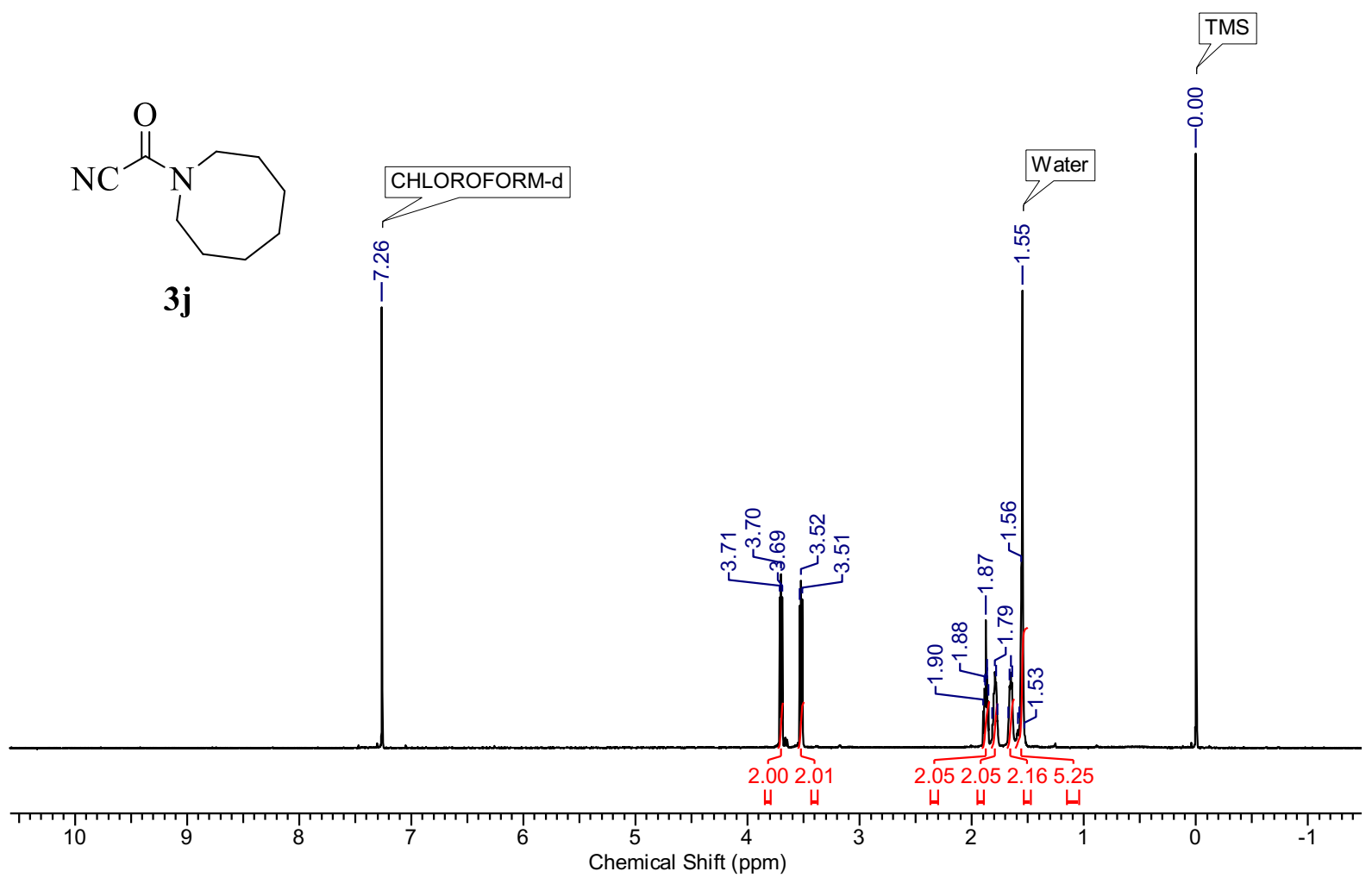

Figure S85. ${ }^{1} \mathrm{H}$ NMR spectrum of $\mathbf{3} \mathbf{j}$ in $\mathrm{CDCl}_{3}(500 \mathrm{MHz})$.

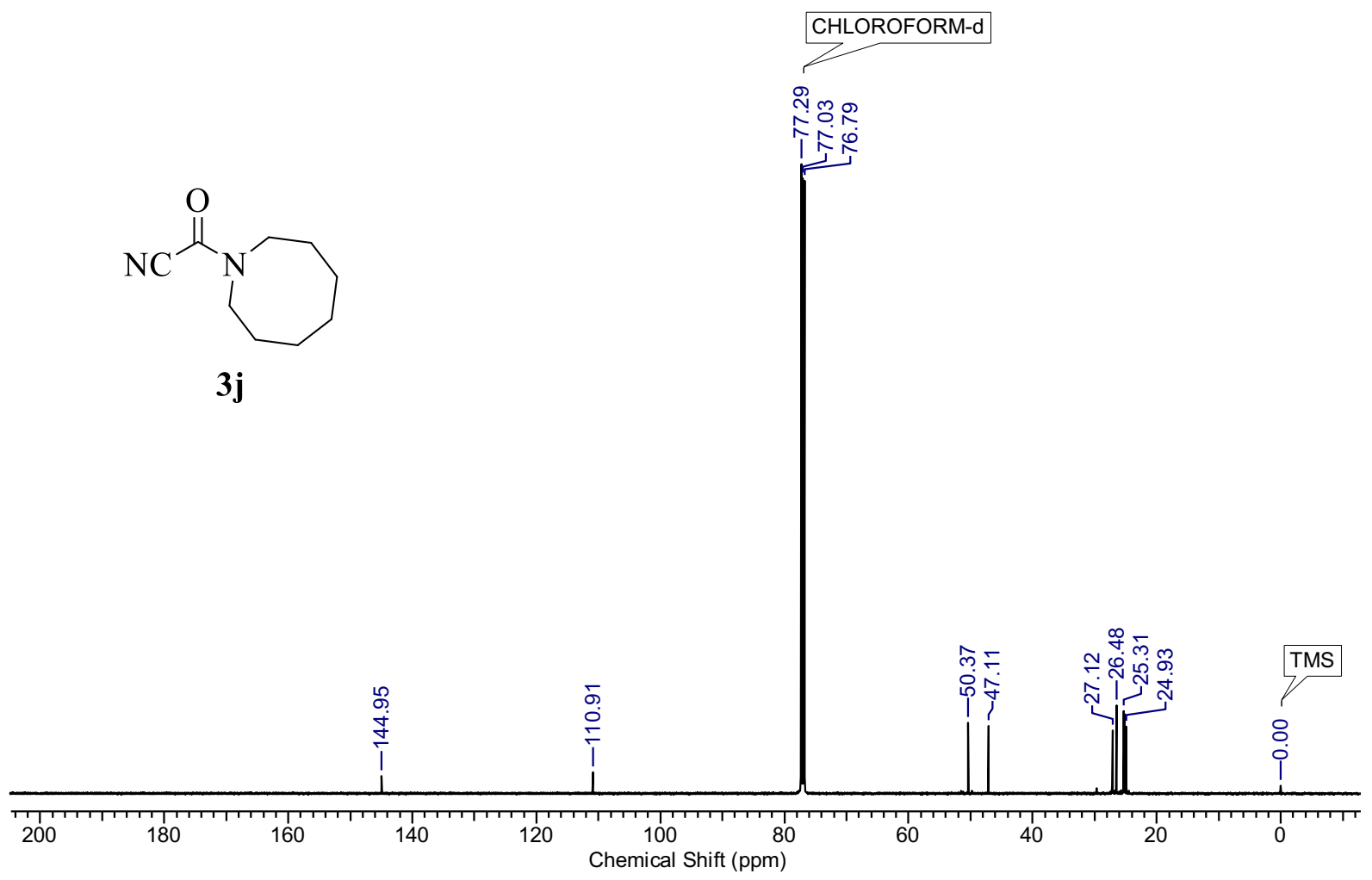

Figure S86. ${ }^{13} \mathrm{C}\left\{{ }^{1} \mathrm{H}\right\}$ NMR spectrum of $\mathbf{3 j}$ in $\mathrm{CDCl}_{3}(125 \mathrm{MHz})$. 
<smiles>N#CC(=O)N1CCCC2CCCCC21</smiles>

$3 k$

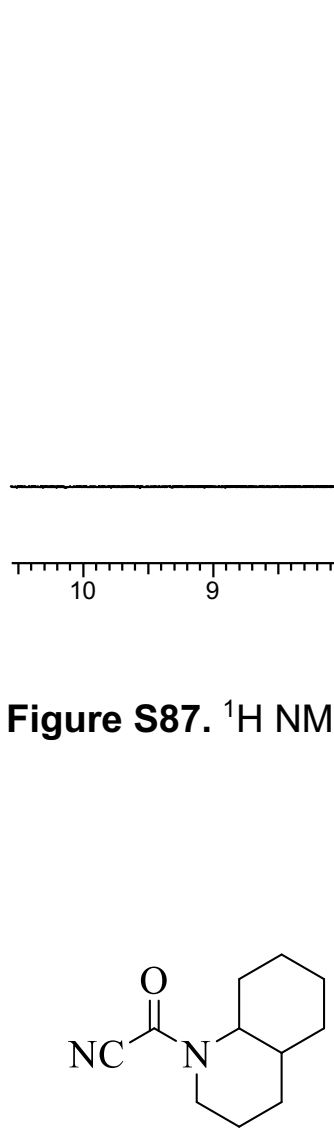

3k

ล

$\stackrel{\Upsilon}{i}$

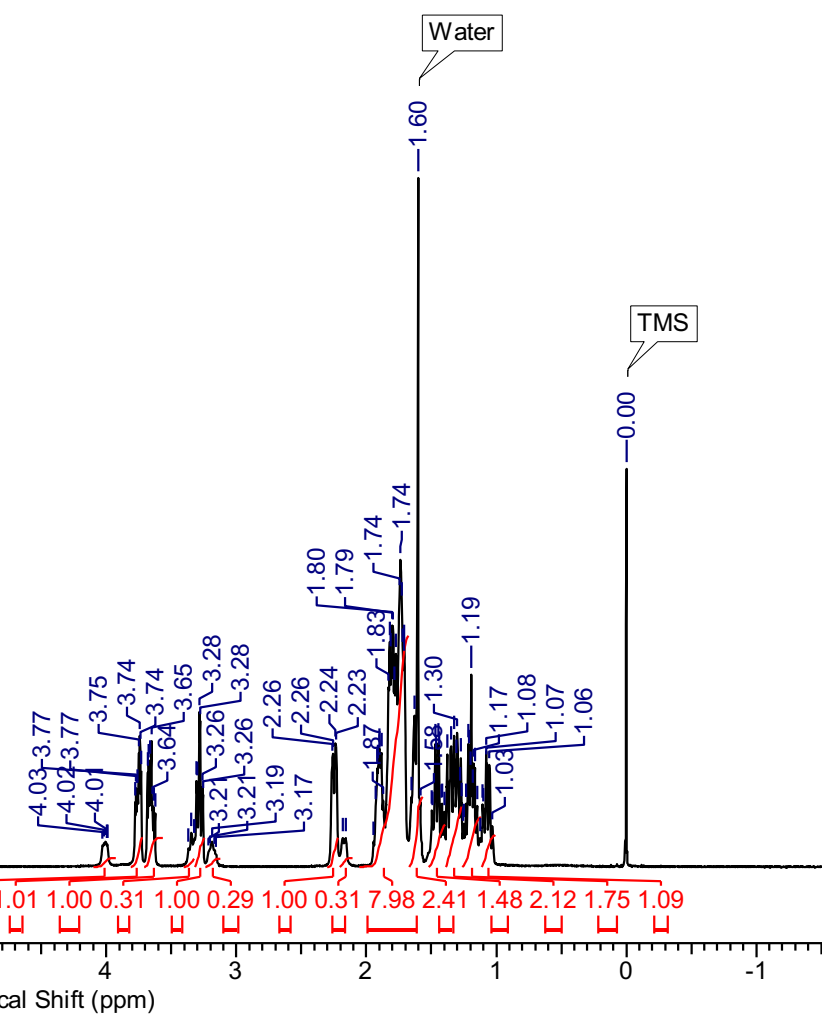

$\begin{array}{cc}5 & 4 \\ \text { Chemical Shift }(p p m)\end{array}$

CHLOROFORM-d

$\mathrm{CDCl}_{3}(500 \mathrm{MHz})$

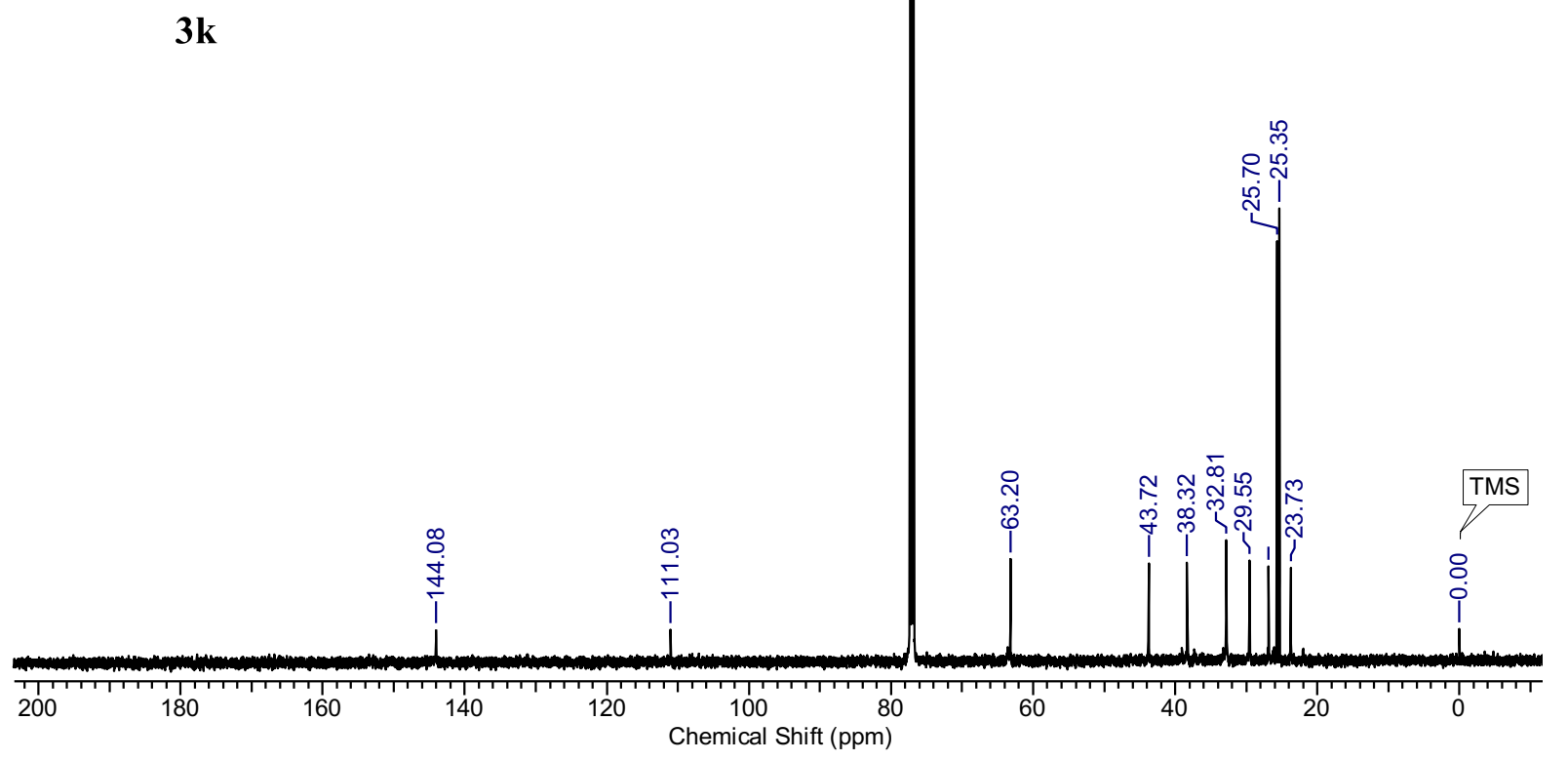

Figure S88. ${ }^{13} \mathrm{C}\left\{{ }^{1} \mathrm{H}\right\}$ NMR spectrum of $\mathbf{3 k}$ in $\mathrm{CDCl}_{3}(125 \mathrm{MHz})$. 
<smiles>N#CC(=O)N1CCC2(CC1)OCCO2</smiles>

31

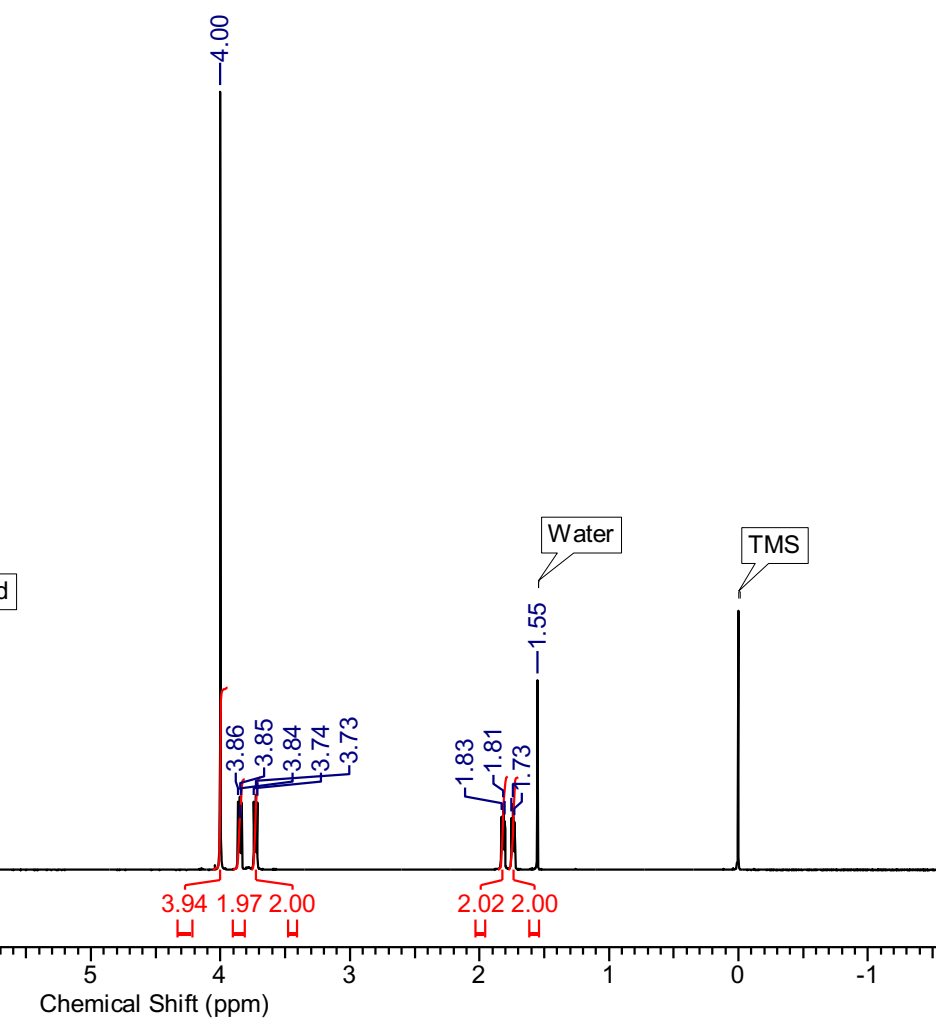

Figure S89. ${ }^{1} \mathrm{H}$ NMR spectrum of $3 \mathrm{I}$ in $\mathrm{CDCl}_{3}(500 \mathrm{MHz})$.

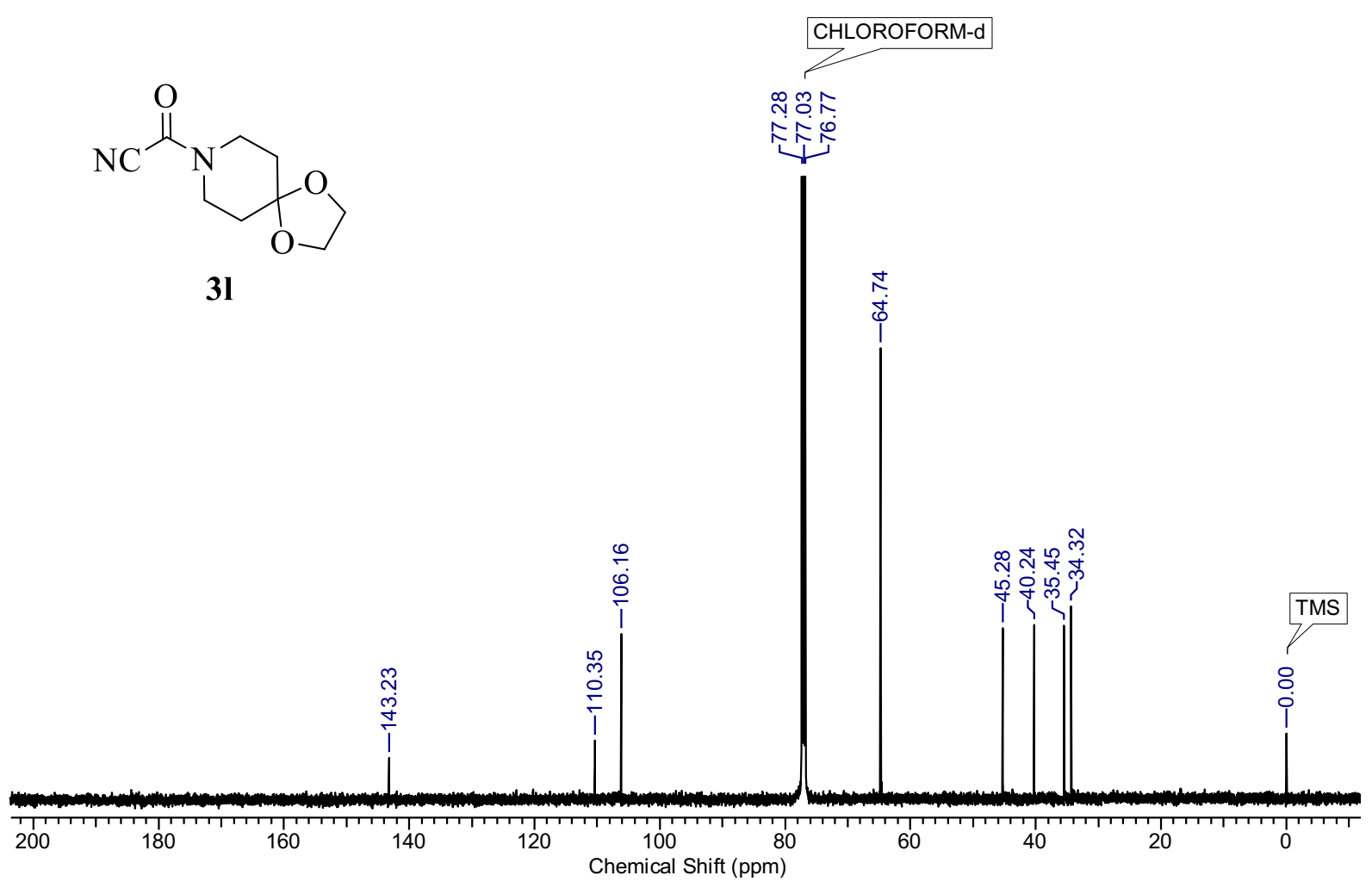

Figure S90. ${ }^{13} \mathrm{C}\left\{{ }^{1} \mathrm{H}\right\}$ NMR spectrum of $3 \mathrm{I}$ in $\mathrm{CDCl}_{3}(125 \mathrm{MHz})$. 


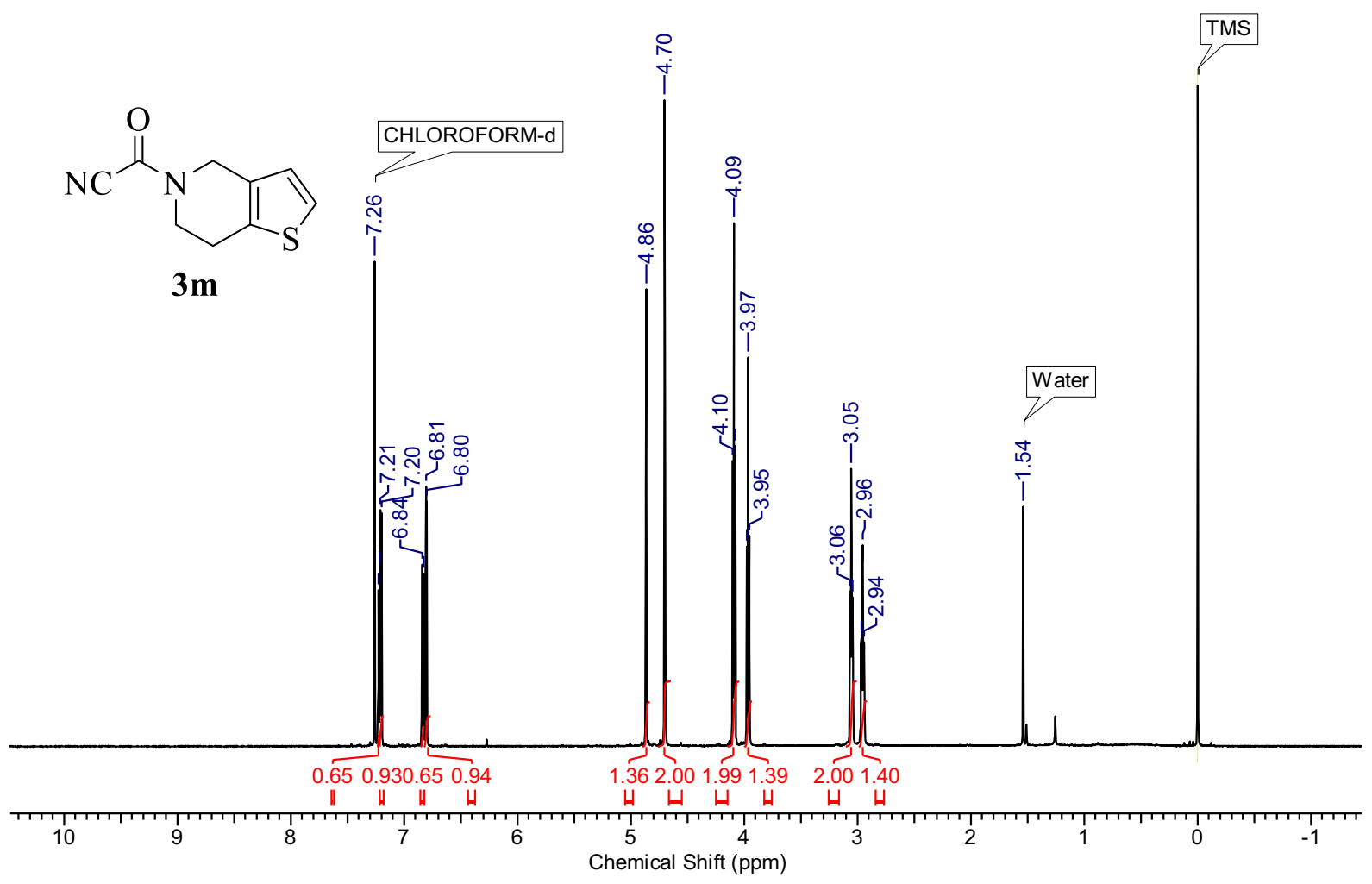

Figure S91. ${ }^{1} \mathrm{H}$ NMR spectrum of $3 \mathrm{~m}$ in $\mathrm{CDCl}_{3}(500 \mathrm{MHz})$.

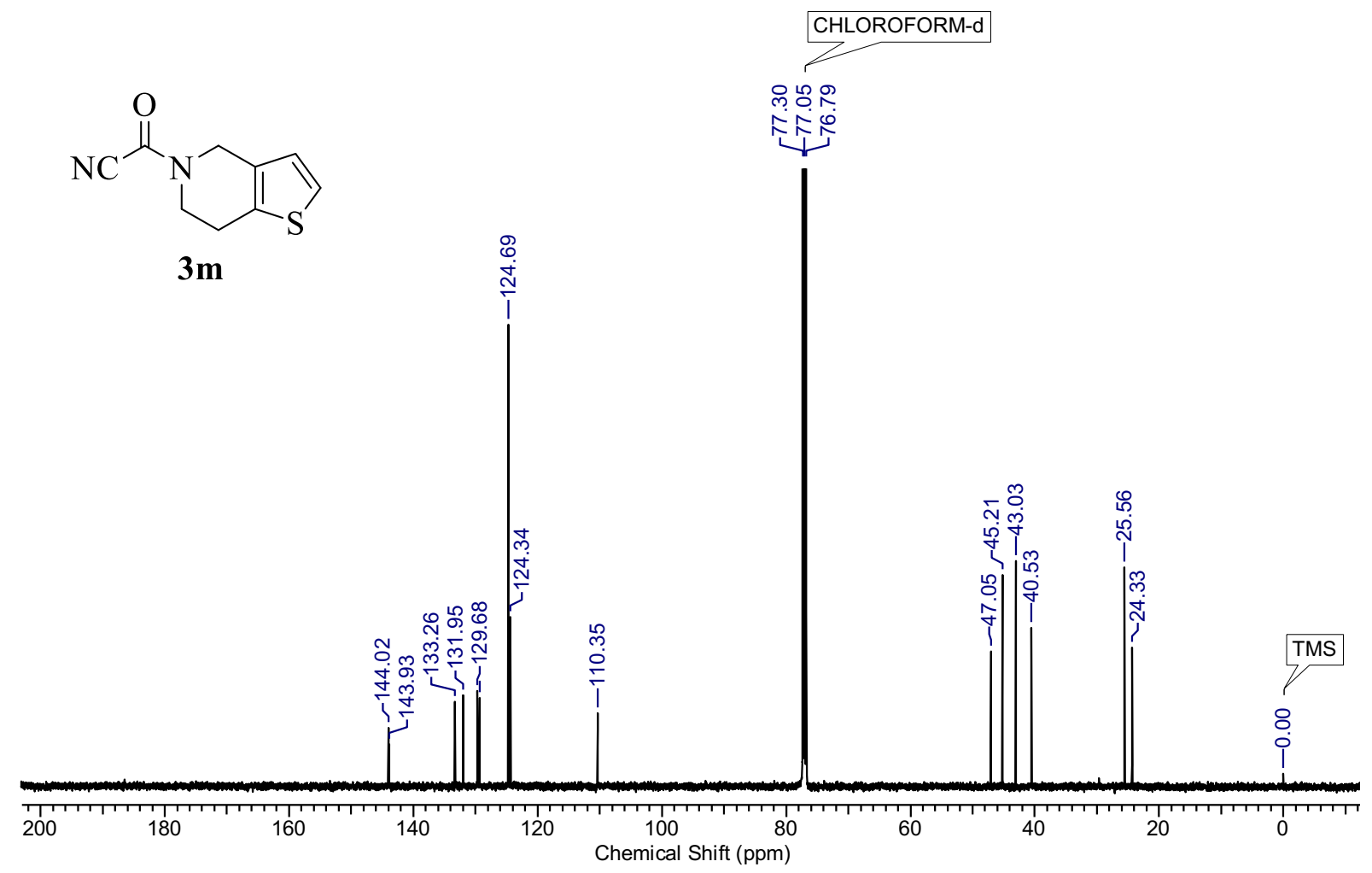

Figure S92. ${ }^{13} \mathrm{C}\left\{{ }^{1} \mathrm{H}\right\}$ NMR spectrum of $3 \mathrm{~m}$ in $\mathrm{CDCl}_{3}(125 \mathrm{MHz})$. 


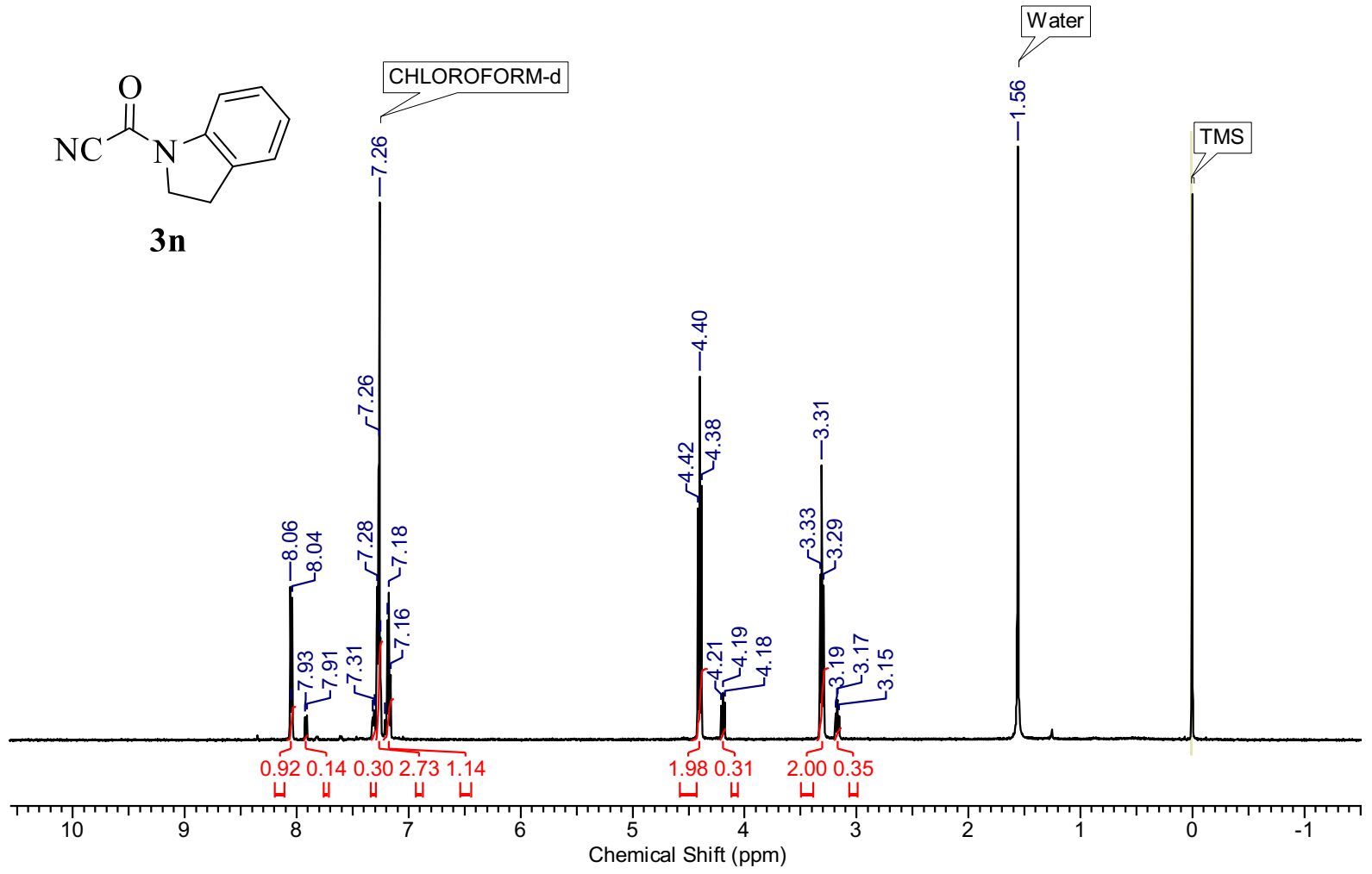

Figure S93. ${ }^{1} \mathrm{H}$ NMR spectrum of $3 n$ in $\mathrm{CDCl}_{3}(500 \mathrm{MHz})$.

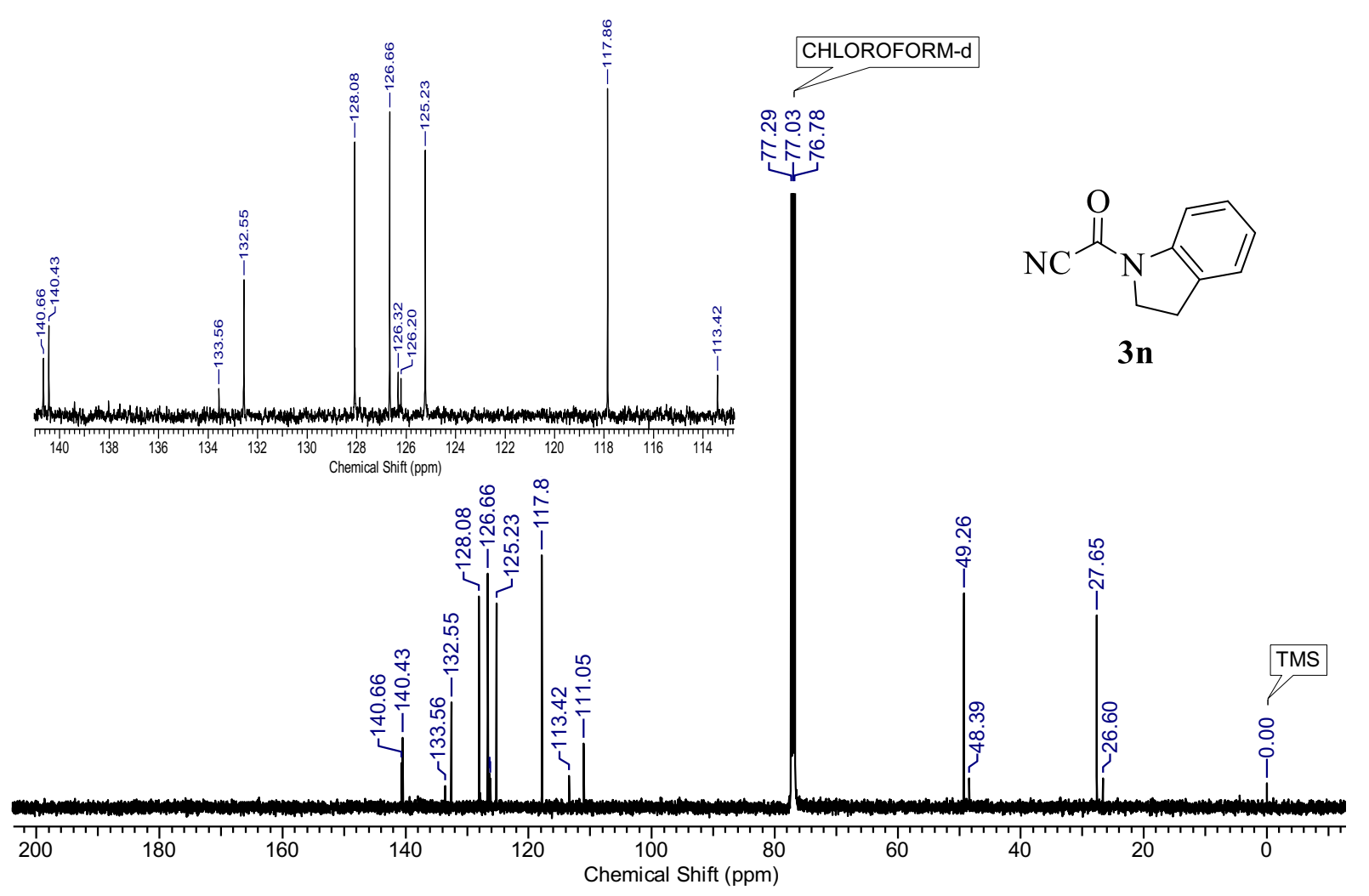

Figure S94. ${ }^{13} \mathrm{C}\left\{{ }^{1} \mathrm{H}\right\}$ NMR spectrum of $3 \mathbf{n}$ in $\mathrm{CDCl}_{3}(125 \mathrm{MHz})$. 


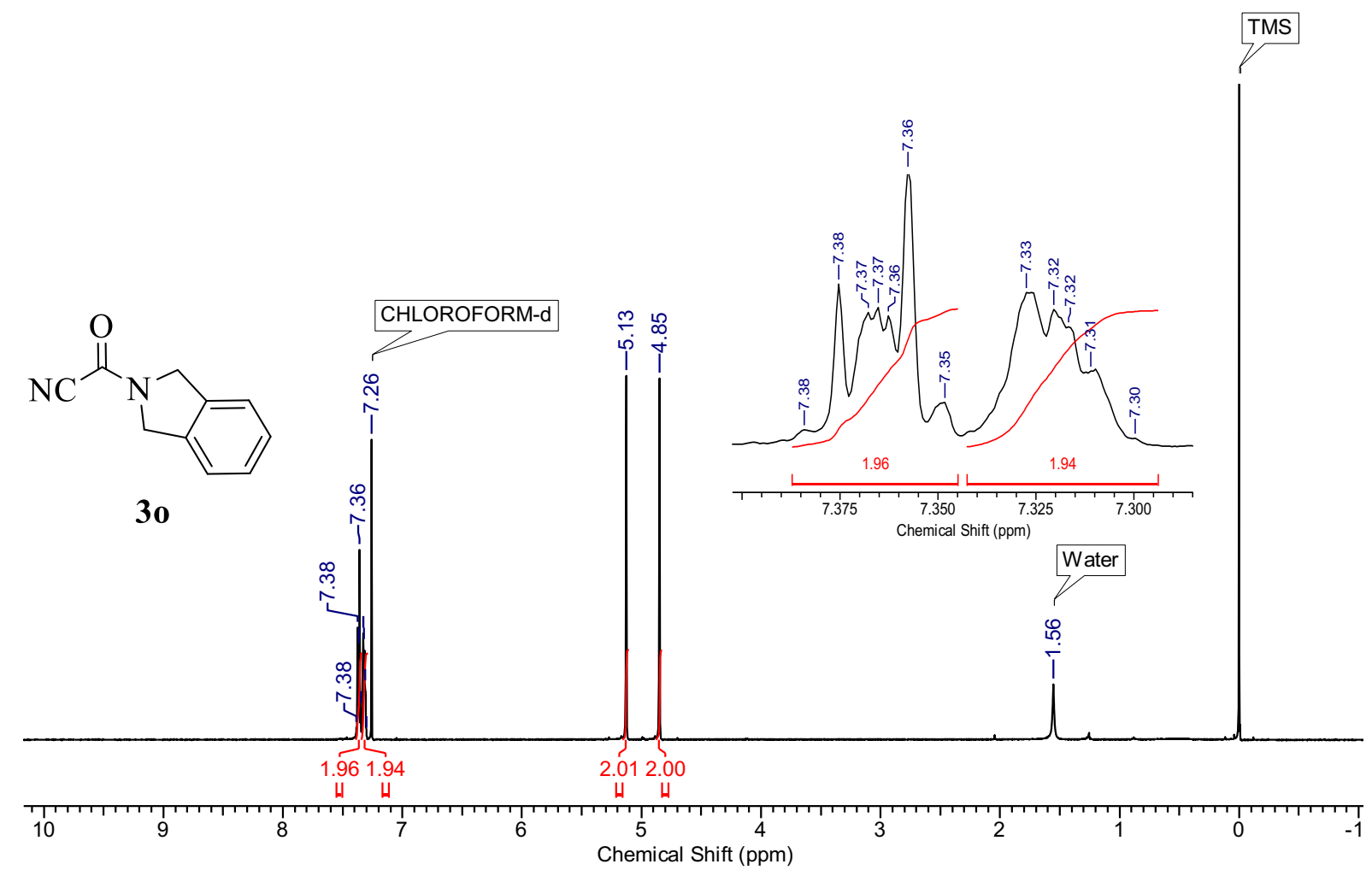

Figure S95. ${ }^{1} \mathrm{H}$ NMR spectrum of 30 in $\mathrm{CDCl}_{3}(500 \mathrm{MHz})$.

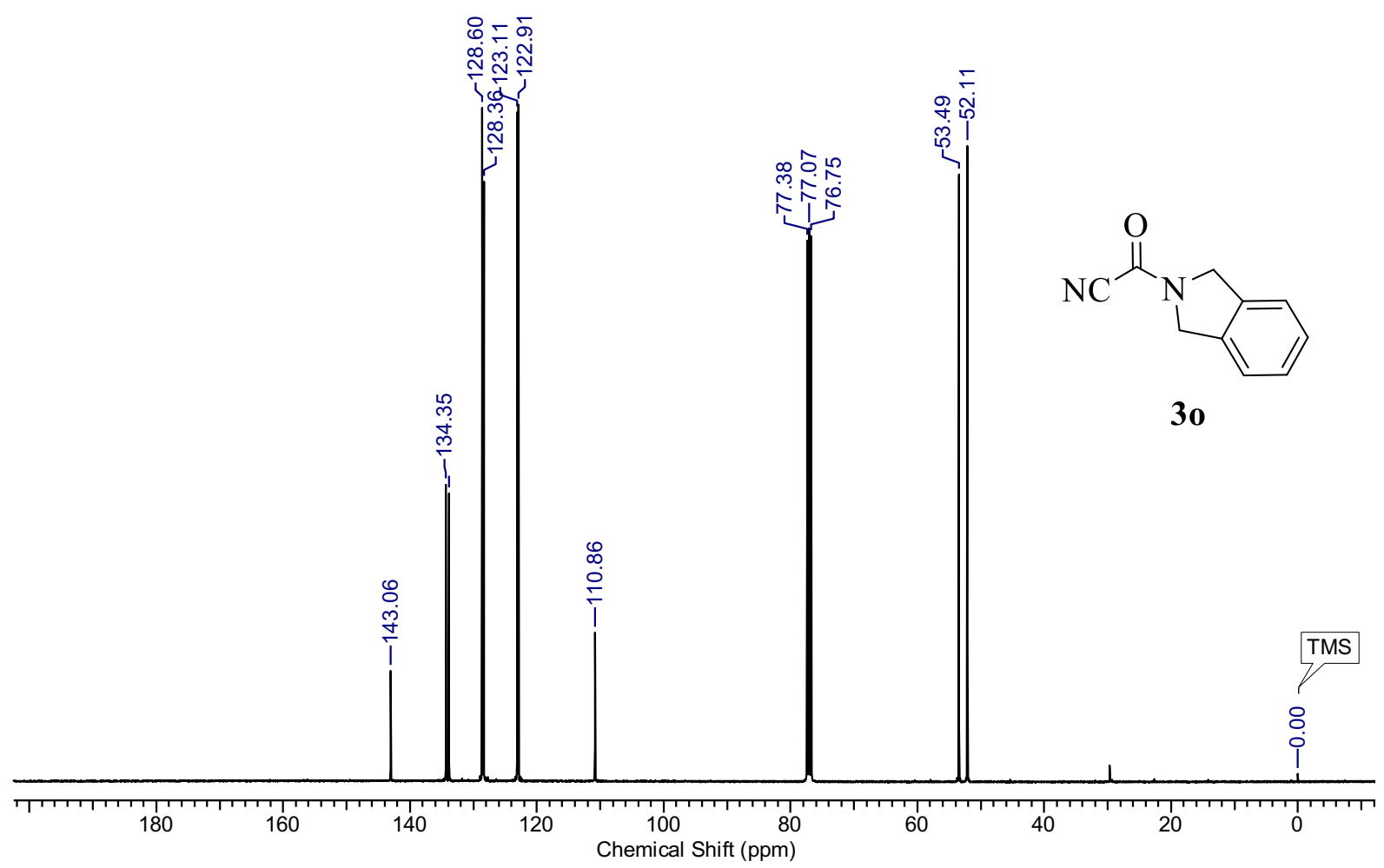

Figure $\mathbf{S} 96{ }^{13} \mathrm{C}\left\{{ }^{1} \mathrm{H}\right\}$ NMR spectrum of 30 in $\mathrm{CDCl}_{3}(125 \mathrm{MHz})$. 


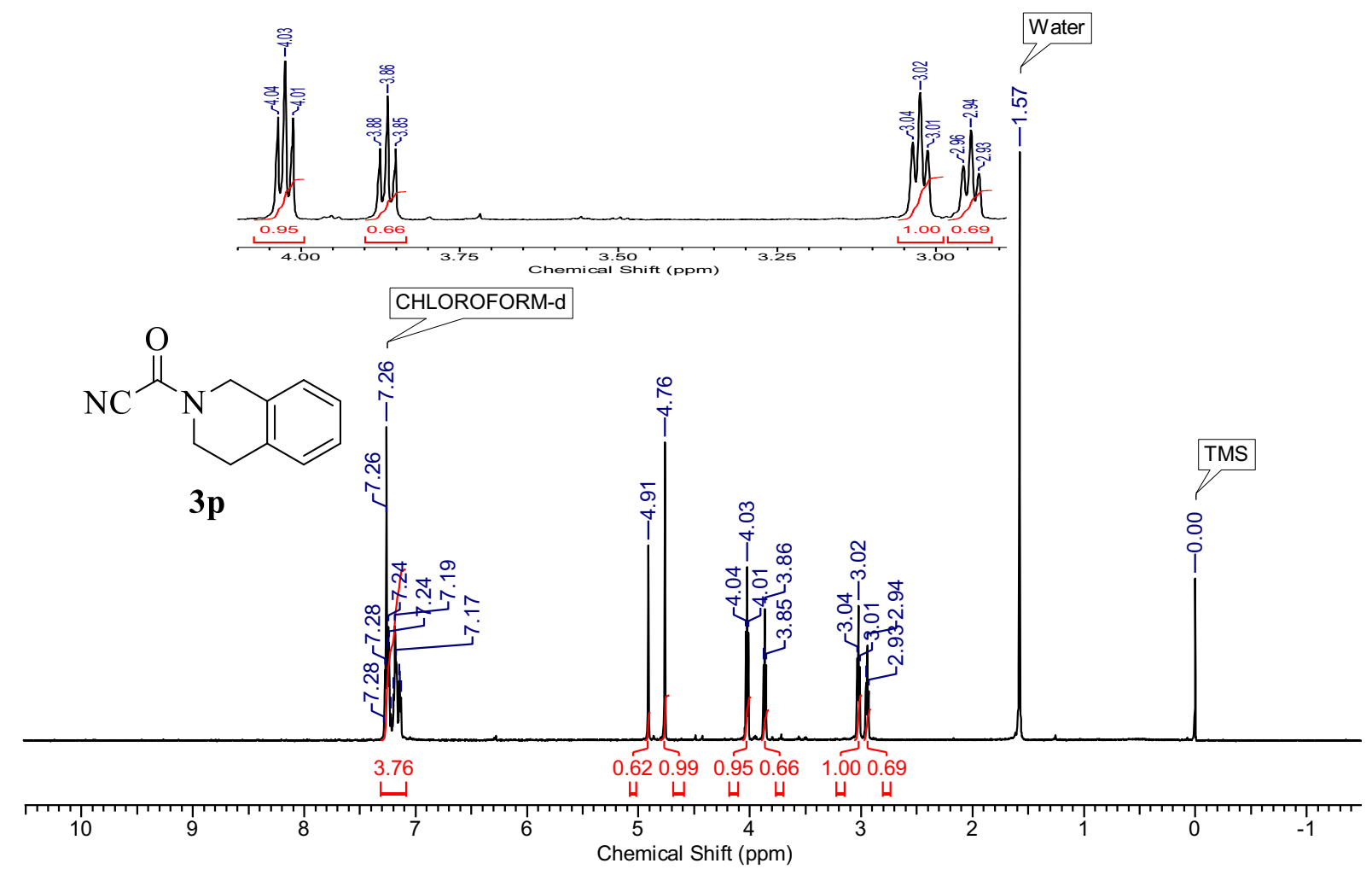

Figure S97. ${ }^{1} \mathrm{H}$ NMR spectrum of $3 p$ in $\mathrm{CDCl}_{3}(500 \mathrm{MHz})$.

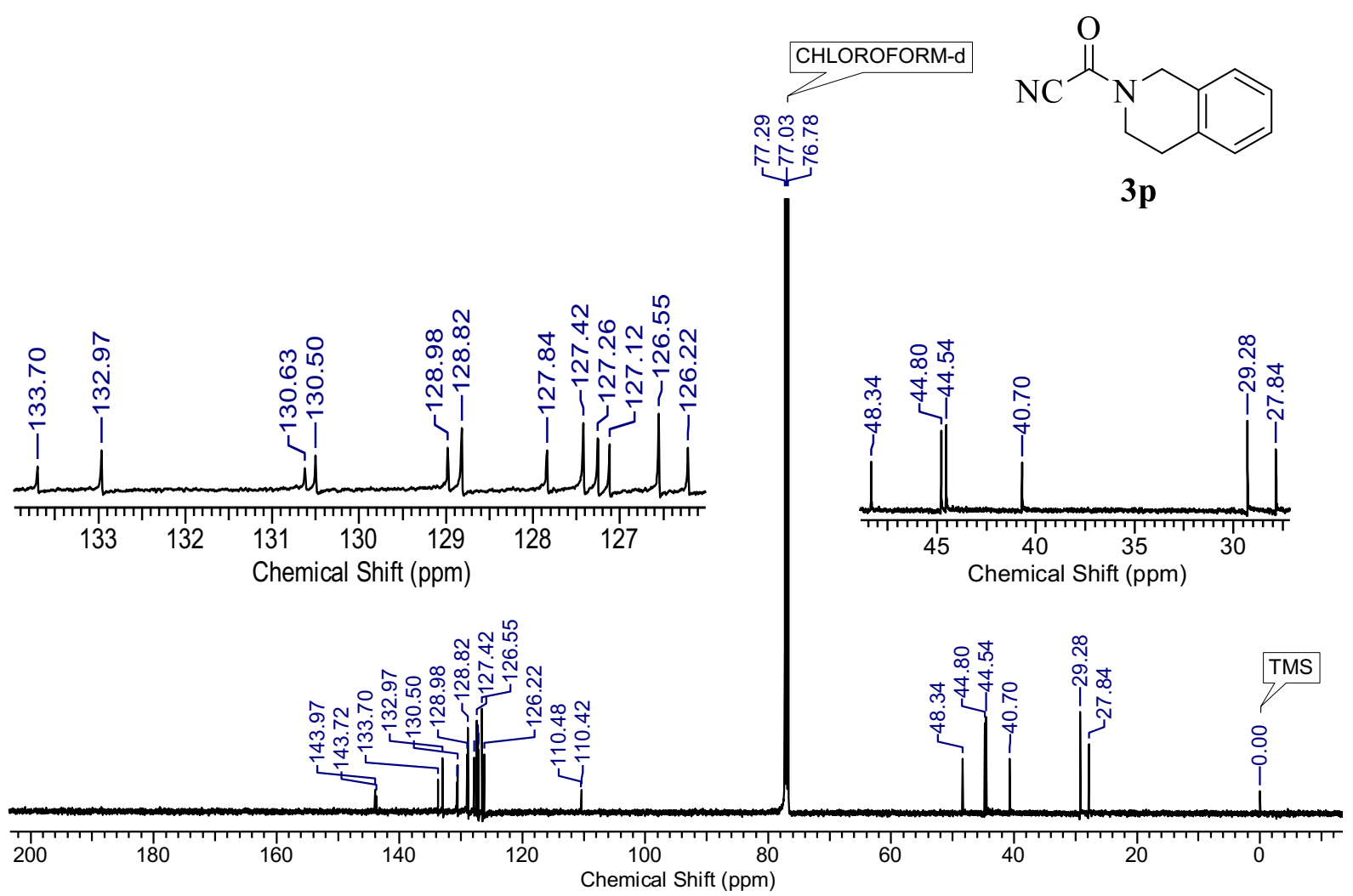

Figure S98. ${ }^{13} \mathrm{C}\left\{{ }^{1} \mathrm{H}\right\}$ NMR spectrum of $3 p$ in $\mathrm{CDCl}_{3}(125 \mathrm{MHz})$. 


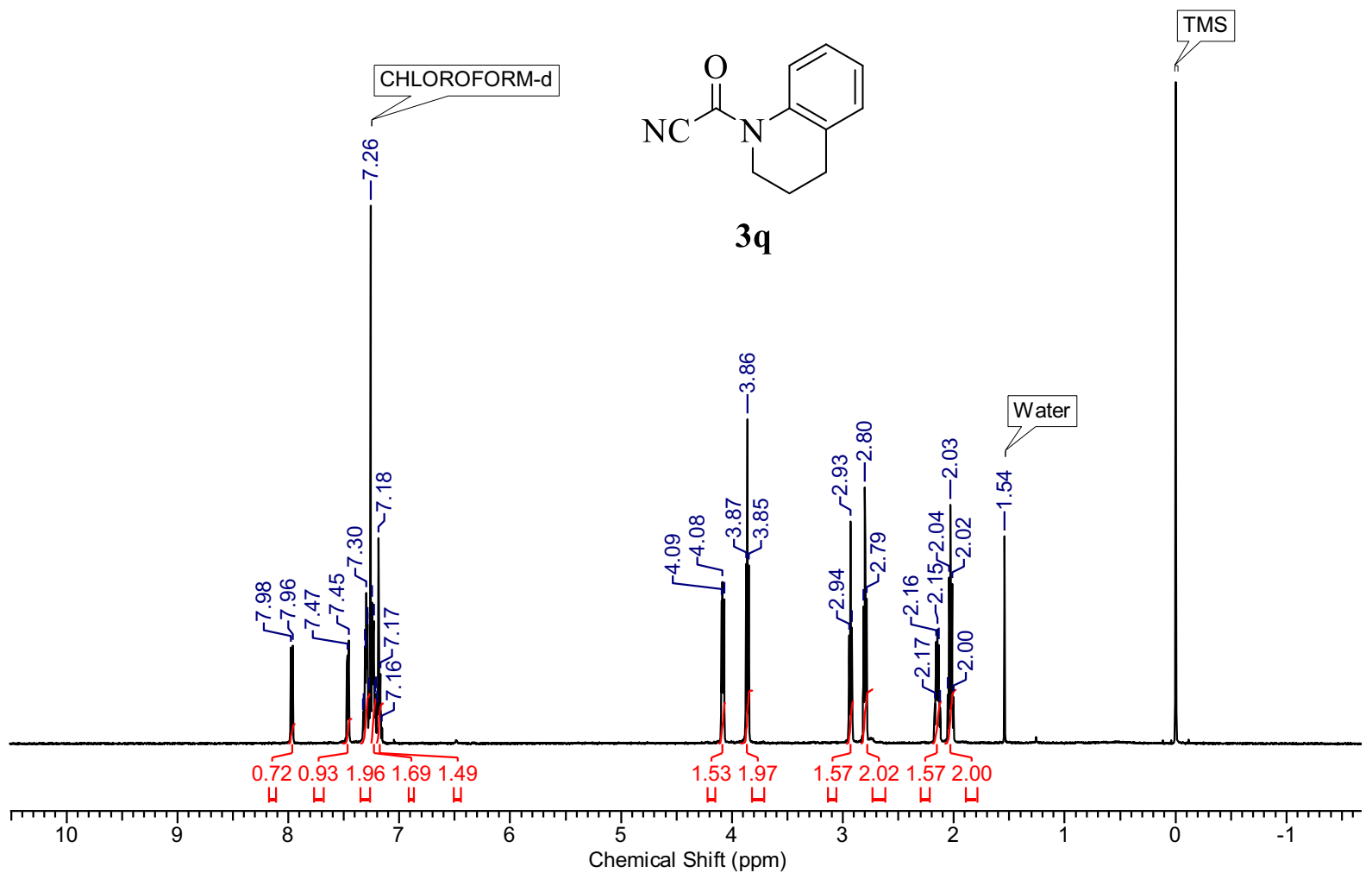

Figure S99. ${ }^{1} \mathrm{H}$ NMR spectrum of $3 q$ in $\mathrm{CDCl}_{3}(500 \mathrm{MHz})$.

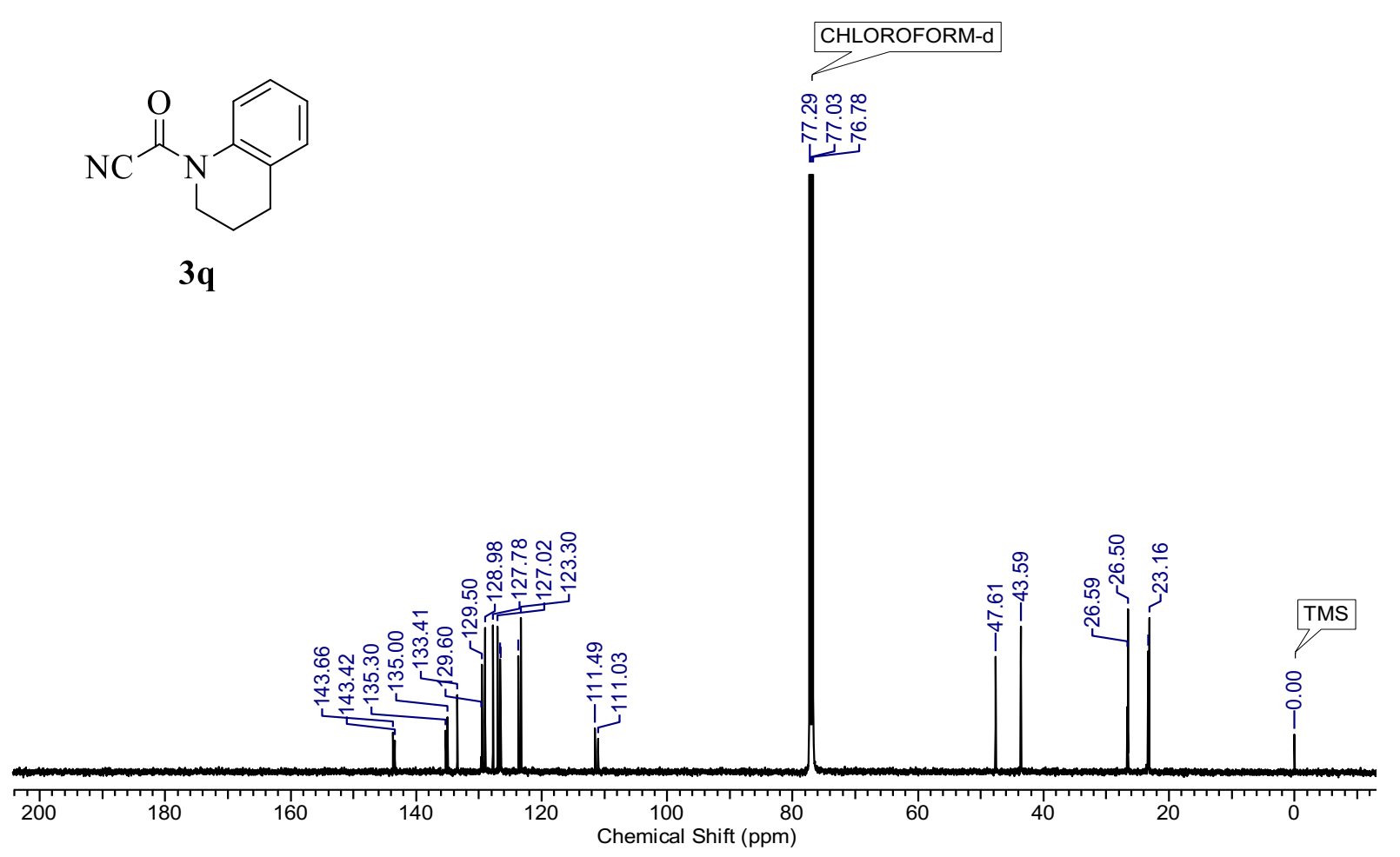

Figure S100. ${ }^{13} \mathrm{C}\left\{{ }^{1} \mathrm{H}\right\}$ NMR spectrum of $\mathbf{3 q}$ in $\mathrm{CDCl}_{3}(125 \mathrm{MHz})$. 


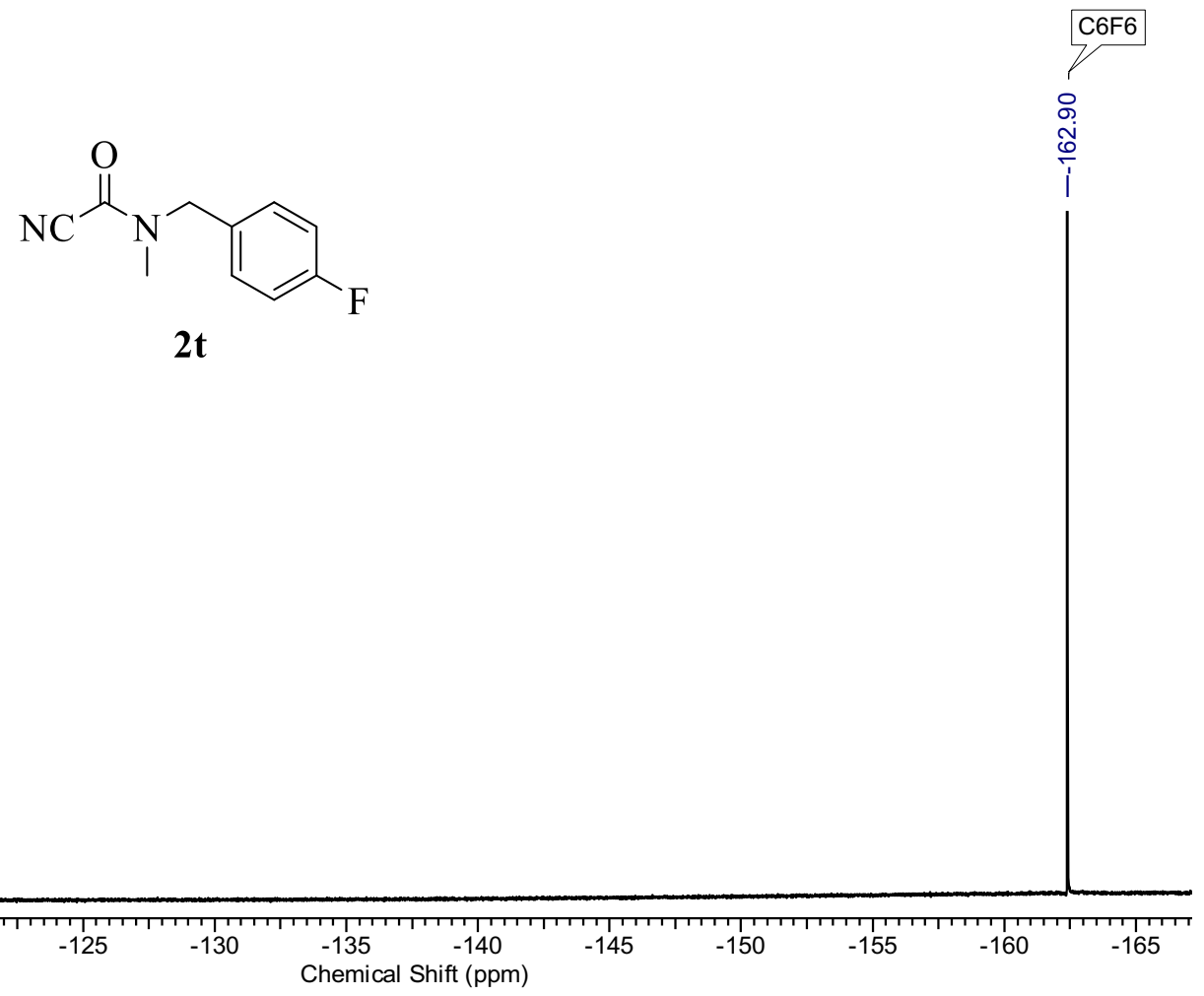

Figure $\mathbf{S} 101 .{ }^{19} \mathrm{~F}$ NMR spectrum of $2 \mathrm{t}$ in $\mathrm{CDCl}_{3}(282 \mathrm{MHz})$.

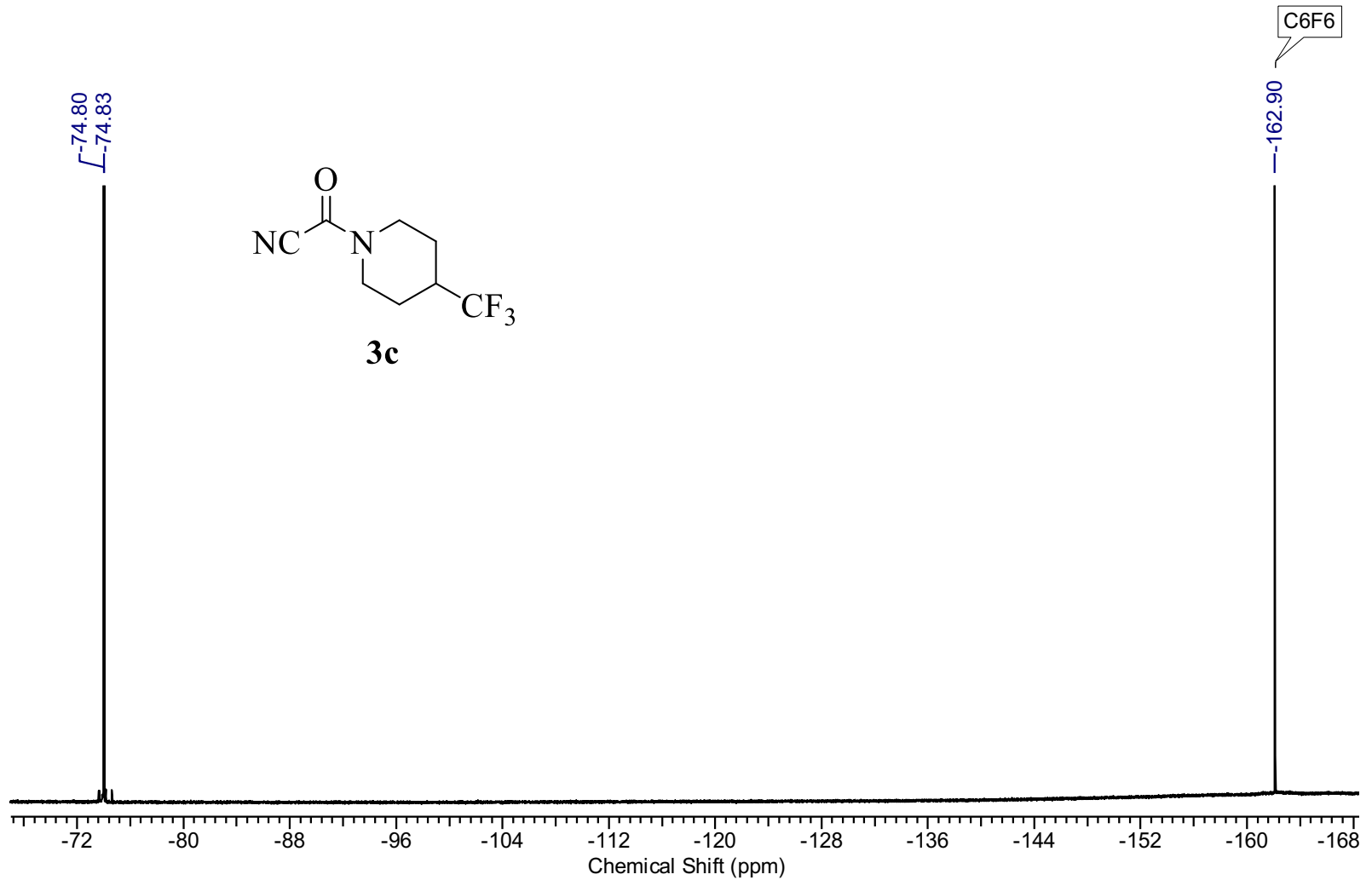

Figure S102. ${ }^{19} \mathrm{~F}$ NMR spectrum of $3 \mathrm{c}$ in $\mathrm{CDCl}_{3}(282 \mathrm{MHz})$. 


\section{References}

1. Tao, J.; Perdew, J. P.; Staroverov, V. N.; Scuseria, G. E. Climbing the Density Functional Ladder: Nonempirical Meta-Generalized Gradient Approximation Designed for Molecules and Solids. Phys. Rev. Lett. 2003, 91, 146401-146404.

2. Grimme, S.; Antony, J.; Ehrlich, S.; Krieg, H. A Consistent and Accurate Ab Initio Parametrization of Density Functional Dispersion Correction (DFT-D) for the 94 Elements H-Pu. J. Chem. Phys. 2010, 132, 154104-154119.

3. Ryde, U.; Mata, R. A.; Grimme, S. Does DFT-D estimate accurate energies for the binding of ligands to metal complexes. Dalton Trans. 2011, 40, 11176-11183.

4. Wachters, A. J. H. Gaussian Basis Set for Molecular Wavefunctions Containing ThirdRow Atoms. J. Chem. Phys. 1970, 52, 1033-1036.

5. Hay, P. J. Gaussian Basis Sets for Molecular Calculations. The Representation of 3d Orbitals in Transition-Metal Atoms. J. Chem. Phys. 1977, 66, 4377-4384.

6. Dunning, T. H., Jr.; Hay, P. J. In Modern Theoretical Chemistry; Schaefer, H. F., III, Ed.; Plenum: New York, 1977; Vol. 3, pp 1-28.

7. Mennucci, B.; Tomasi, J. Continuum Solvation Models: A New Approach to the Problem of Solute's Charge Distribution and Cavity Boundaries. J. Chem. Phys. 1997, 106 , 51515158.

8. Frisch, M. J.; Trucks, G. W.; Schlegel, H. B.; Scuseria, G. E.; Robb, M. A.; Cheeseman, J. R.; Scalmani, G.; Barone, V.; Petersson, G. A.; Nakatsuji, H.; Li, X.; Caricato, M.; Marenich, A.; Bloino, J.; Janesko, B. G.; Gomperts, R.; Mennucci, B.; Hratchian, H. P.; Ortiz, J. V.; Izmaylov, A. F.; Sonnenberg, J. L.; Williams-Young, D.; Ding, F.; Lipparini, F.; Egidi, F.; Goings, J.; Peng, B.; Petrone, A.; Henderson, T.; Ranasinghe, D.; Zakrzewski, V. G.; Gao, J.; Rega, N.; Zheng, G.; Liang, W.; Hada, M.; Ehara, M.; Toyota, K.; Fukuda, R.; Hasegawa, J.; Ishida, M.; Nakajima, T.; Honda, Y.; Kitao, O.; Nakai, H.; Vreven, T.; Throssell, K.; Montgomery, Jr., J. A.; Peralta, J. E.; Ogliaro, F.; Bearpark, M.; Heyd, J. J.; Brothers, E.; Kudin, K. N.; Staroverov, V. N.; Keith, T.; Kobayashi, R.; Normand, J.; Raghavachari, K.; Rendell, A.; Burant, J. C.; lyengar, S. S.; Tomasi, J.; Cossi, M.; Millam, J. M.; Klene, M.; Adamo, C.; Cammi, R.; Ochterski, J. W.; Martin, R. L.; Morokuma, K.; Farkas, O.; Foresman, J. B.; Fox, D. J. Gaussian 09, rev. E.01; Gaussian, Inc.: Wallingford, CT, 2016.

9. CrysAlisPro, Tokyo, Japan, 2015.

10. Sheldrick, G. M. SHELXT - Integrated Space-Group and Crystal-Structure Determination. Acta Crystallogr. Sect. A Found. Crystallogr. 2015, 71, 3-8.

11. Sheldrick, G. M. Crystal Structure Refinement with SHELXL. Acta Crystallogr. Sect. C Struct. Chem. 2015, 71, 3-8. 
12. Dolomanov, O. V.; Bourhis, L. J.; Gildea, R. J.; Howard, J. A. K.; Puschmann, H. OLEX2: A Complete Structure Solution, Refinement and Analysis Program. J. Appl. Crystallogr. 2009, 42, 339-341.

13. Florence, T. M.; Farrar, Y. J. Spectrophotometric Determination of Chloride at the Partsper-Billion Level by the Mercury(II) Thiocyanate Method. Anal. Chim. Acta 1971, 54, 373377. 